\title{
Large Deviations in Weakly Interacting Fermions - Generating Functions as Gaussian Berezin Integrals and Bounds on Large Pfaffians
}

\author{
N. J. B. Aza \\ J.-B. Bru \\ W. de Siqueira Pedra \\ L. C. P. A. M. Müssnich
}

May 27, 2021

\begin{abstract}
We prove that the Gärtner-Ellis generating function of probability distributions associated with KMS states of weakly interacting fermions on the lattice can be written as the limit of logarithms of Gaussian Berezin integrals. The covariances of the Gaussian integrals are shown to have a uniform Pfaffian bound and to be summable in general cases of interest, including systems that are not translation invariant. The Berezin integral representation can thus be used to obtain convergent expansions of the generating function in terms of powers of its parameter. The derivation and analysis of the expansions of logarithms of Berezin integrals are the subject of the second part of the present work. Such technical results are also useful, for instance, in the context of quantum information theory, in the computation of relative entropy densities associated with fermionic Gibbs states, and in the theory of quantum normal fluctuations for weakly interacting fermion systems.
\end{abstract}

Keywords: Berezin integral, constructive methods, interacting fermions, determinant bound, large deviations.

AMS Subject Classification: 81T08, 82C22, 46L53

\section{Contents}

1 Introduction $\quad 2$

2 Self-dual CAR Algebra and Bilinear Hamiltonians 9

2.1 Self-dual CAR Algebra . . . . . . . . . . . . . . . . . . . . . 9

2.2 Bilinear Elements of Self-dual CAR Algebra . . . . . . . . . . . . . . . 11

2.3 Quasi-Free Dynamics and States . . . . . . . . . . . . . . . . . . 13

3 Realization of Self-dual CAR Algebra as a Grassmann Algebra 15

3.1 Grassmann Algebra . . . . . . . . . . . . . . . . . . . . . . . . . . . . . . . . . . . .

3.2 Berezin Integral . . . . . . . . . . . . . . . . . . . . . 16

3.3 Bilinear Elements of Grassmann Algebra _ . . . . . . . . . . . . . . . . 18

3.4 From Grassmann Algebra to Self-Dual CAR Algebra . . . . . . . . . . . . . . . 21

4 Generating Functions as Berezin Integrals 2

4.1 The Berezin Integral Representation of the Tracial State . . . . . . . . . . . . . . . 26

4.2 Chernoff Product Formula . . . . . . . . . . . . . . . . . 28

4.3 Feynman-Kac-like Formula for Generating Functions . . . . . . . . . . . . . 30 
5 Properties of the Covariance $\quad 32$

5.1 Pfaffian Bounds . . . . . . . . . . . . . . . . . . . 32

5.2 Covariances as Correlations of Quasi-Free States . . . . . . . . . . . . . 36

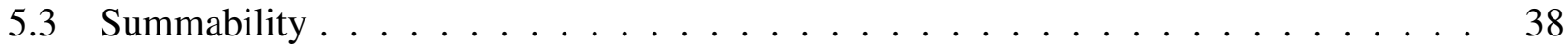

5.3.1 Lattice Fermion Systems in the Self-dual Formalism . . . . . . . . . . . 38

5.3.2 Combes-Thomas Estimate and Summability of the Fermi Distribution . . . . 38

5.3.3 Summability of Covariances - General Bound . . . . . . . . . . . . . . 40

5.3.4 Summability of Covariances - Gapped Case . . . . . . . . . . . . . . . . . 42

6 CAR Algebra and Second Quantization 44

6.1 CAR Algebra . . . . . . . . . . . . . . . . . . . . . . . . . 44

6.2 From CAR Algebra to Self-Dual CAR Algebra . . . . . . . . . . . . . . . . 45

6.3 Quadratic Fermionic Hamiltonians as Bilinear Elements . . . . . . . . . . . . . 45

6.4 Fock Representation of CAR Algebra . . . . . . . . . . . . . . . . . . . . 46

7 Quantum Large Deviations $\quad 48$

7.1 Large Deviations (LD) in Quantum Statistical Mechanics . . . . . . . . . . . . . . 48

7.2 LD Formalism for Quantum Observables . . . . . . . . . . . . . . . . . . . 50

8 Application of LD to Quantum Statistical Mechanics 52

8.1 Spin or CAR Algebra . . . . . . . . . . . . . . . . . . . . . 52

8.2 Thermodynamic Sequences of Observables . . . . . . . . . . . . . . 53

8.3 Exponentially Ergodic States . . . . . . . . . . . . . . . . . . . . . 54

8.4 Thermal Equilibrium States for Fermion Systems _ . . . . . . . . . . . . . 55

\section{Introduction}

This paper is the first step towards large deviations (LD) analyses of fermions that, at equilibrium, weakly interact with each other on lattices. Various fields of theoretical physics and mathematics are concerned in this study and, to make our results understandable to a wider audience, we describe their mathematical framework in detail in Sections 7-8, including a historical overview (Section 7.1). Indeed, LD methods have been used many times to study quantum systems from at least the 1980s.

To begin, we review recent LD results related to the present work. In 2000, Lebowitz, Lenci and Spohn proved [LLS00] that the particle density of an ideal $d$-dimensional continuum quantum (Fermi or Bose) gas at thermal equilibrium obeys a so-called large deviation principle (LDP), cf. [LLS00, Theorem II.1] and Section 7.2. This is proven for arbitrary inverse temperatures $\beta \in \mathbb{R}^{+}$and chemical potentials $\mu$. There is no interparticle interaction and the system is supposed to be in a single phase, which is guaranteed by imposing differentiability of the pressure [LLS00, p. 1226]. For convergence, a clustering condition is assumed.

Two years later, Gallavotti, Lebowitz and Mastropietro [GLM02] contributed another proof that the particle density of (rarefied) quantum gases fulfills an LDP at thermal equilibrium. The authors consider systems of particles satisfying either Boltzmann, Bose, or Fermi statistics, but we focus here on results for lattice Fermi systems. In this case, the proof is done for weakly interacting fermions via Berezin integrals ${ }^{1}$ and tree expansions for correlation functions of the equilibrium state (cf. [GLM02, Equation (7.11)]), from which the (limiting) logarithmic moment generating function (Section 7.2) is obtained. This approach is strongly connected to the one we develop presently. The complete proof of the LDP in [GLM02] may be achieved by getting bounds for large determinants (or Pfaffians) associated with the full covariance (cf. [GLM02, Expression (7.23)]), which is, nonetheless, not

\footnotetext{
${ }^{1}$ Also called Grassmann or Grassmann-Berezin integrals.
} 
done by the authors. Instead, they perform a multiscale decomposition of the covariance to bound the resulting series of determinants afterwards. See [GLM02, Item (7) on page 855]. Avoiding the multiscale analysis is one of our main motivations here. We also show that it is not necessary to use correlation functions to construct the (limiting) logarithmic moment generating function $\mathrm{J}$ via functional integrals:

- We directly write $\mathrm{J}$ as a Gaussian Berezin integral with a covariance that differs from the one for correlation functions. See below Equations (9)-(10).

- Then, we prove that this covariance is summable and satisfies a Pfaffian bound in the desired way.

In contrast to [GLM02], the method presented here, which will be heavily exploited in [BdSPX], is not restricted to particle density observables $\left\{\mathrm{D}_{l}\right\}_{l \in \mathbb{R}^{+}}(139)$. It can be applied to any thermodynamic sequences $\left\{\mathrm{E}_{l}^{\Psi}\right\}_{l \in \mathbb{R}^{+}}(141)$ associated with densities of any other physical quantity that can be encoded in a translation-invariant and finite-range interaction $\Psi$ (see Section 8.2). Before explaining in more detail our technical approach in relation to the one of [GLM02], we proceed with the review of recent LD results in quantum statistical mechanics.

In 2004, Netočný and Redig [NR04] studied quantum spin systems at thermal equilibrium. They demonstrated the existence of an LDP and a central limit theorem at sufficiently high temperatures for thermodynamic sequences $\left\{\mathrm{E}_{l}^{\Psi}\right\}_{l \in \mathbb{R}^{+}}$of density observables encoded in zero-range $e^{2}$ interactions $\Psi$. This result thus holds true for more general thermodynamic sequences than just the particle density case used in previous studies. Note that the central limit theorem in [NR04] is a consequence of the Bryc theorem (Theorem 7.2), which will also be used in [BdSPX].

The following year, Lenci and Rey-Bellet [LR05] took into consideration empirical means (space averages) $\left\{\mathrm{M}_{l}^{A}\right\}_{l \in \mathbb{R}^{+}}$of a finite-volume element $A$ in either spin or CAR $C^{*}$-algebra, as defined below by (138). This quantity refers to thermodynamic sequences $\left\{\mathrm{E}_{l}^{\Psi}\right\}_{l \in \mathbb{R}^{+}}$of density observables for more general translation-invariant, finite-range interactions $\Psi$, as defined below by (141). See also Equation (142). In contrast to [NR04], they did not exploit cluster expansions, but the inequality

$$
\left|\ln \operatorname{tr}\left(A \mathrm{e}^{H+K}\right)-\ln \operatorname{tr}\left(A \mathrm{e}^{H}\right)\right| \leq \sup _{0 \leq t \leq 1} \sup _{-\frac{1}{2} \leq w \leq \frac{1}{2}}\left\|\mathrm{e}^{-w(H+t K)} K \mathrm{e}^{w(H+t K)}\right\|_{\mathcal{B}\left(\mathbb{C}^{n}\right)},
$$

for any matrices $A, H, K$ so that $A>0, H=H^{*}$ and $K=K^{*}$, where tr denotes the normalized trace. See [LR05, Lemma 3.6]. $\mathcal{B}(\mathcal{X})$ denotes the space of linear operators acting on a vector space $\mathcal{X}$. The uniqueness of the thermal equilibrium state, i.e., the Kubo-Martin-Schwinger (KMS) state, is used as a key argument, in contrast to [NR04] (cf. Remark 8.4 and [NR04, Remark 2.14]). They obtain an LDP for the empirical average of finite-volume observables either in dimension one at any temperature, or for any finite dimension at sufficiently high temperatures. Their proof works for both spin and fermionic lattice systems. See [LR05, Theorem 3.2].

Note that the bound (1) is only useful if its right-hand side behaves as $\|K\|_{\mathcal{B}\left(\mathbb{C}^{n}\right)}$, uniformly with respect to the choices of the self-adjoint matrices $H$ and $K$ in $\mathcal{B}\left(\mathbb{C}^{n}\right)$. This is related to the following open problem: to find sufficient conditions on an interaction $\Phi$, as defined in Section 8.2, such that, for fixed $A$ in (finite-volume) spin or CAR $C^{*}$-algebra, the norm of $\mathrm{e}^{H_{L}^{\Phi}} A \mathrm{e}^{-H_{L}^{\Phi}}$ does not diverge in the thermodynamic limit $L \rightarrow \infty$. Here, as defined below by Equation (148), $H_{L}^{\Phi}$ is the Hamiltonian associated with the interaction $\Phi$ in some cubic box $\Lambda_{L}$ of side length $L \in \mathbb{R}^{+}$. Araki [A69] proved this non-divergence for all translation-invariant, finite-range interactions in the one-dimensional case. Nevertheless, the assertion was recently proven to be false in dimension two [Bo11], at least for sufficiently large $|\Phi|$. One of the advantages of constructing the logarithmic moment generating

\footnotetext{
${ }^{2}$ A zero-range interaction $\Psi$ yields a sequence of the form $\mathrm{E}_{l}^{\Psi}=\frac{1}{\left|\Lambda_{l}\right|} \sum_{x \in \Lambda_{l}} \Psi_{\{x\}}$, which is a particular case of the sequences defined by Equation (141). See Section 8.2.
} 
function via Gaussian Berezin integrals, together with the corresponding tree expansion we will use in $[\mathrm{BdSPX}]$, is that such uniform bounds are not needed, at least not explicitly.

In 2011, Ogata and Rey-Bellet [OR11] made use of the Ruelle-Lanford function [Ru65, L73] to technically simplify and unify the proof of previous LD results for quantum spin systems with a slight extension: Their proofs are based on the notion of "asymptotic decoupled states" as defined in the classical case in [P02, Definitions 3.2 and 3.3], i.e., states $\rho$ on spin $C^{*}$-algebra for which there is a function $c: \mathbb{N} \rightarrow[0, \infty)$ such that, for any $L \in \mathbb{N}$, and any non-negative observables $A, B$ with corresponding supports $\Lambda^{(A)} \subset \Lambda_{L}$ and $\Lambda^{(B)} \subset \mathbb{Z}^{d} \backslash \Lambda_{L}$,

$$
\lim _{L \rightarrow \infty} \frac{c(L)}{\left|\Lambda_{L}\right|}=0 \quad \text { and } \quad \mathrm{e}^{-c(L)} \rho(A) \rho(B) \leq \rho(A B) \leq \mathrm{e}^{c(L)} \rho(A) \rho(B) .
$$

There are few quantum systems for which KMS states are known to satisfy this condition, for instance at dimension one [A69] and at high temperatures [A74], like the cases considered in [NR04, LR05].

More recently, De Roeck, Maes, Netočný and Schütz studied in [dRMNS15] (identically distributed (i.d.)) empirical means of operators associated with zero-range interactions for quantum spin systems at thermal equilibrium, via the corresponding distributions as defined below by (130). They focus on Gibbsianness or quasi-locality of the (limiting) distribution, which is a stronger feature than the property of asymptotic decoupling used in [OR11], which in turn is stronger than an LD property. See, e.g., [dRMNS15, Theorem 4.4]. Like in [NR04, LR05, OR11] they study the high temperature regime, but also the low temperature situation for a class of gapped quantum spin systems. In all studied regimes, there is a unique KMS state. They use a polymer model together with either a high-temperature cluster expansion or an expansion around the ground state that is reminiscent of the quantum Pirogov-Sinai theory.

In the case of weakly interacting fermions on lattices, we aim to show both an LDP and a central limit theorem for the translation-invariant thermal equilibrium states at any temperature and any dimension for any thermodynamic sequences $\left\{\mathrm{E}_{l}^{\Psi}\right\}_{l \in \mathbb{R}^{+}}(141)$ that is encoded in a translation-invariant and finite-range interaction $\Psi$. The first technical step towards this result is performed in the current paper.

We study fermion systems in their algebraic formulation via self-dual CAR algebra [EK98, Chap. 6], a concept first introduced by Araki in [A68]. Araki's view point does not seem to have strongly percolated into the general mathematical physics community ${ }^{3}$, even though it can be used to elegantly treat non-gauge invariant quadratic models. Therefore, the preliminary discussions of the results are restricted to the well-known CAR algebra setting. Below, $\operatorname{CAR}(\mathfrak{h})$ denotes the CAR $C^{*}$-algebra associated with any finite-dimensional Hilbert space $\mathfrak{h}$, like for fermion systems on a finite lattice.

Using the tracial state tr on $\operatorname{CAR}(\mathfrak{h})$, i.e., the normalized trace on the finite-dimensional fermionic Fock space representation (Section 6.4, in particular Remark 6.4), we explain in Section 4.3 how the quantity

$$
\operatorname{tr}\left(\mathrm{e}^{-\beta \mathbf{H}} \mathrm{e}^{s K}\right), \quad \beta \in \mathbb{R}^{+}, s \in \mathbb{R} \quad \text { and } \quad \mathbf{H}=\mathbf{H}^{*}, K=K^{*} \in \mathrm{CAR}(\mathfrak{h})
$$

can be written as a limit of Berezin integrals. This is related to a version of the Feynman-Kac formula proven here. See Theorem 4.6 and Remark 4.7.

We consider fermionic Hamiltonians of the form

$$
\mathbf{H}=\mathrm{d} \Gamma(h)+\mathrm{d} \Upsilon(g)+W, \quad W=W^{*} \in \mathrm{CAR}(\mathfrak{h}),
$$

\footnotetext{
${ }^{3}$ For instance, the term "self-dual CAR algebra" does not even appear in the celebrated textbook [BR96b]. It is only implicitly there in the discussion of Bogoliubov transformations at the end of [BR96b, Section 5.2.2.1].
} 
where, for any $h=h^{*} \in \mathcal{B}(\mathfrak{h})$ and antilinear operator $g=-g^{*}$ on $\mathfrak{h}$,

$$
\begin{aligned}
\mathrm{d} \Gamma(h) & \doteq \sum_{i, j \in J}\left\langle\psi_{i}, h \psi_{j}\right\rangle_{\mathfrak{h}} a\left(\psi_{i}\right)^{*} a\left(\psi_{j}\right), \\
\mathrm{d} \Upsilon(g) & \doteq \frac{1}{2} \sum_{i, j \in J}\left(\left\langle\psi_{i}, g \psi_{j}\right\rangle_{\mathfrak{h}} a\left(\psi_{i}\right)^{*} a\left(\psi_{j}\right)^{*}+\overline{\left\langle\psi_{i}, g \psi_{j}\right\rangle_{\mathfrak{h}}} a\left(\psi_{j}\right) a\left(\psi_{i}\right)\right) .
\end{aligned}
$$

Here, $\left\{\psi_{j}\right\}_{j \in J}$ is any orthonormal basis ${ }^{4}$ of $\mathfrak{h}$, and $a(\varphi), a(\varphi)^{*} \in \mathrm{CAR}(\mathfrak{h})$ are the usual fermionic annihilation/creation operators of a fermion in the state $\varphi \in \mathfrak{h}$. As is usual, $h^{*}$ and $g^{*}$ are the adjoints ${ }^{5}$ of $h$ and $g$, respectively.

The self-adjoint, even element $\mathrm{d} \Gamma(h) \in \mathrm{CAR}(\mathfrak{h})$ is the second quantization of the one-particle Hamiltonian $h \in \mathcal{B}(\mathfrak{h})$. It represents a gauge invariant model of free fermions. The non-gauge invariant quadratic part of $\mathbf{H}$ is represented by the self-adjoint and even element $\mathrm{d} \Upsilon(g) \in \mathrm{CAR}(\mathfrak{h})$. Such "off-diagonal" terms appear, for instance, in the celebrated BCS theory of superconductivity. Note that it is well-known [B66, A68] that quadratic fermionic Hamiltonians like $\mathrm{d} \Gamma(h)+\mathrm{d} \Upsilon(g)$ can always be transformed via some (Bogoliubov) unitary transformation into $\mathrm{d} \Gamma(\tilde{h})+\lambda$ for some constant $\lambda \in \mathbb{R}$ and matrix $\tilde{h} \in \mathcal{B}(\mathfrak{h})$. Such a transformation is, in general, not explicitly given and also changes the element $W$, which encodes in (3) the interparticle interaction of the fermion system. It is, however, technically advantageous to avoid such a transformation: Instead of modifying the model, we adapt our Grassmann-algebra construction. In this context, the self-dual CAR framework turns out to be much more natural than the usual CAR setting.

If the element $W$ is taken to be an even element of $\operatorname{CAR}(\mathfrak{h})$, like for any many-fermion model in physics, then we show that (2) can be written in terms of a limit of Gaussian Berezin integrals in the following sense:

$$
\frac{\operatorname{tr}\left(\mathrm{e}^{-\beta(\mathrm{d} \Gamma(h)+\mathrm{d} \Upsilon(g)+W)} \mathrm{e}^{s K}\right)}{\operatorname{tr}\left(\mathrm{e}^{-\beta(\mathrm{d} \Gamma(h)+\mathrm{d} \Upsilon(g))}\right)}=\lim _{n \rightarrow \infty} \int \mathrm{d} \mu_{C_{h, g}^{(n)}}\left(\mathfrak{H}^{(n)}\right) \mathrm{e}^{\mathcal{W}_{W, s K}^{(n)}}
$$

Inside this limit,

$$
\int \mathrm{d} \mu_{C_{h, g}^{(n)}}\left(\mathfrak{H}^{(n)}\right)
$$

stands for a Gaussian Berezin integral on some Grassmann algebra over $\mathfrak{H}^{(n)}$ associated with an explicit covariance $C_{h, g}^{(n)}$ only dependent on $h, g, \beta$ and $n \in \mathbb{N}$. $\mathcal{W}_{W, s K}^{(n)}$ is the result of a canonical mapping from $\operatorname{CAR}(\mathfrak{h})$ to the Grassmann algebra over $\mathfrak{H}^{(n)}$ and only depends on $W, s K, \beta$ and $n \in \mathbb{N}$. For more details, see Section 4.3, in particular Corollary 4.8.

In the future application to LDP for lattice fermion systems at equilibrium, $\beta$ is the inverse temperature and $s \in \mathbb{R}$ will be the parameter of the logarithmic moment generating function. For $L_{\mathrm{f}} \geq L_{\mathrm{i}} \geq l \geq 0$, we take $\mathfrak{h}=\ell^{2}\left(\Lambda_{L_{\mathrm{f}}}\right)$ where $\Lambda_{L_{\mathrm{f}}} \subset \mathbb{Z}^{d}(d \in \mathbb{N})$ is a centered cubic box of side length $\mathcal{O}\left(L_{\mathrm{f}}\right)$, see Section 8.1 and Equation (99). The elements $W=\left|\Lambda_{L_{\mathrm{i}}}\right| \mathrm{E}_{L_{\mathrm{i}}}^{\Phi_{\mathrm{i}}}$ and $K=\left|\Lambda_{l}\right| \mathrm{E}_{l}^{\Psi}$ will be associated with two arbitrary translation-invariant, finite-range interactions $\Phi_{\mathrm{i}}$ and $\Psi$, respectively, as defined below by (141). In this case, Equation (6) allows us to write the logarithmic moment generating function

$$
\mathrm{J}_{L_{\mathrm{f}}, L_{\mathrm{i}}, l}^{\Psi}(s) \doteq \frac{1}{\left|\Lambda_{l}\right|} \ln \frac{\operatorname{tr}\left(\mathrm{e}^{-\beta\left(\mathrm{d} \Gamma(h)+\mathrm{d} \Upsilon(g)+\left|\Lambda_{L_{\mathrm{i}}}\right| \mathrm{E}_{L_{\mathrm{i}}}^{\Phi_{\mathrm{i}}}\right)} \mathrm{e}^{s\left|\Lambda_{l}\right| \mathrm{E}_{l}^{\Psi}}\right)}{\operatorname{tr}\left(\mathrm{e}^{-\beta\left(\mathrm{d} \Gamma(h)+\mathrm{d} \Upsilon(g)+\left|\Lambda_{L_{\mathrm{i}}}\right| \mathrm{E}_{L_{\mathrm{i}}}^{\Phi_{\mathrm{i}}}\right)}\right)},
$$

\footnotetext{
${ }^{4} \mathrm{~d} \Gamma(h), \mathrm{d} \Upsilon(g)$ are self-adjoint elements which do not depend on the choice of the orthonormal basis, but on the choice of generators $\{a(\varphi)\}_{\varphi \in \mathfrak{h}}$ of $\operatorname{CAR}(\mathfrak{h})$.

${ }^{5}$ The adjoint of an antilinear map $g$ is defined by the condition $\left\langle\varphi_{1}, g^{*} \varphi_{2}\right\rangle_{\mathfrak{h}}=\left\langle\varphi_{2}, g \varphi_{1}\right\rangle_{\mathfrak{h}}, \varphi_{1}, \varphi_{2} \in \mathfrak{h}$.
} 
associated with $\left\{\mathrm{E}_{l}^{\Psi}\right\}_{l \in \mathbb{R}^{+}}$in the Gibbs state, as the limit of Gaussian Berezin integrals for any inverse temperature $^{6} \beta \in \mathbb{R}^{+}, s \in \mathbb{R}$ and $L_{\mathrm{f}} \geq L_{\mathrm{i}} \geq l \geq 0$.

We intend to use in [BdSPX] the so-called Brydges-Kennedy tree expansion to show, at any dimension $d \geq 1$ and any fixed inverse temperature ${ }^{7} \beta$, the existence of an analytic continuation to a centered disk in the complex plane of the logarithmic moment generating function

$$
\mathrm{J}^{\Psi}(s) \doteq \lim _{l \rightarrow \infty} \lim _{L_{\mathrm{i}} \rightarrow \infty} \lim _{L_{\mathrm{f}} \rightarrow \infty} \mathrm{J}_{L_{\mathrm{f}}, L_{\mathrm{i}}, l}^{\Psi}(s)
$$

associated with $\left\{\mathrm{E}_{l}^{\Psi}\right\}_{l \in \mathbb{R}^{+}}$and any weak* accumulation point ${ }^{8}$ of the sequence of Gibbs states of weakly interacting fermions on lattices. Our method thus applies to systems for which the uniqueness of the KMS state is not known. As explained in Section 7.2, such a result yields an LDP and a central limit theorem for the corresponding observables.

\section{Remark 1.1}

The method we set forth here (and later in [BdSPX]) can be used to study relative entropy densities [JOPP12, Section 2.6] and quantum normal fluctuations [V11, Section 6] for fermion systems on lattices. It can also be useful in the theory of "quantum hypothesis testing" via Chernoff and Hoefding bounds. See for instance [HMO07, Section VI]. For more explanations, see [BdSPX].

The convergence of the Brydges-Kennedy tree expansion in non-relativistic fermionic constructive quantum field theory is ensured at weak interaction, provided that (i) Pfaffians arising in the expansion can be efficiently bounded and (ii) the covariance is summable:

(i) In Section 5.1 we provide (up to an exponential term with small rate) sharp bounds on Pfaffians of fermionic covariances $C_{h, g}^{(n)}$ appearing in (6). These bounds are uniform with respect to the choice of operators $h=h^{*}, g=-g^{*}$ and the dimension of $\mathfrak{h}$. No translation invariance is required.

(ii) In Section 5.2 we explain how one obtains the summability of the covariance $C_{h, g}^{(n)}$ from the decay of the two-point correlation functions. In Section 5.3, we prove the summability of the covariance at any inverse temperature $\beta \in \mathbb{R}^{+}$for general lattice fermion systems. In the gapped case, the covariance is proven to be uniformly summable with respect to $\beta \in \mathbb{R}^{+}$, as generally expected and already verified in various similar cases considered in the literature. The proofs are based on the celebrated Combes-Thomas bound [AW16, Theorem 10.5]. In particular, the translation invariance is again not required for the summability of the covariance.

In contrast to (ii), observe that the Pfaffians associated with fermionic covariances are not deduced from the corresponding Pfaffians or determinants for two-point correlations. Indeed, the covariances $C_{h, g}^{(n)}, n \in \mathbb{N}$, appearing in (6) are related to a non-autonomous difference equation: To simplify the discussion ${ }^{9}$, assume that $g=0$. Let $\ell_{\text {ap }}^{2}\left(\mathbb{T}_{n} ; \mathfrak{h}\right)$ be the space of antiperiodic $\mathfrak{h}$-valued functions on the discrete torus

$$
\mathbb{T}_{n} \doteq\left\{\left(k-n_{\beta}+1\right) n^{-1} \beta: k \in\left\{0, \ldots, 2 n_{\beta}-1\right\}\right\} \subset(-\beta-1, \beta+1]
$$

\footnotetext{
${ }^{6}$ The case $\beta=0$ corresponds to use the trace state instead of the Gibbs state and is not considered here. It can be studied by much simpler methods since, in this case, the logarithmic moment generating function is a pressure.

${ }^{7}$ More precisely, $\beta$ must not be small, as in most previous results for $d>1$, but rather the interparticle interaction $\Phi_{\mathrm{i}}$ has to be small enough, depending on $\beta$.

${ }^{8}$ The function $\mathrm{J}^{\Psi}$ a priori depends on the weak* accumulation point of Gibbs states, fixed by the choice of a subsequence of $L_{\mathrm{f}} \geq L_{\mathrm{i}} \geq 0$.

${ }^{9}$ The general case can be mapped to the special case $g=0$ by unitary transformations.
} 
with $n_{\beta} \doteq n+\lfloor n / \beta\rfloor,\lfloor x\rfloor$ being the largest natural number smaller than $x \in \mathbb{R}^{+}$. For any matrix $h=h^{*} \in \mathcal{B}(\mathfrak{h})$, define $\mathfrak{u}_{h} \in \mathcal{B}\left(\ell_{\text {ap }}^{2}\left(\mathbb{T}_{n} ; \mathfrak{h}\right)\right)$ by

$$
\left[\mathfrak{u}_{h} f\right](\alpha) \doteq \begin{cases}h(f(\alpha)) & \text { for } \quad \alpha \in \mathbb{T}_{n} \cap(0, \beta] \\ 0 & \text { for } \quad \alpha \in \mathbb{T}_{n} \cap(\beta, \beta+1]\end{cases}
$$

for any $f \in \ell_{\text {ap }}^{2}\left(\mathbb{T}_{n} ; \mathfrak{h}\right)$. Denote by $\partial \in \mathcal{B}\left(\ell_{\text {ap }}^{2}\left(\mathbb{T}_{n} ; \mathfrak{h}\right)\right)$ the discrete time derivative operator. Then, the discrete time covariance used to define the corresponding Gaussian Berezin integrals for $g=0$ is equal to

$$
C_{h, 0}^{(n)}=-2\left(\partial+\mathfrak{u}_{h}\right)^{-1} \in \mathcal{B}\left(\ell_{\mathrm{ap}}^{2}\left(\mathbb{T}_{n} ; \mathfrak{h}\right)\right) .
$$

This representation of covariances is not used further in this paper. It is nonetheless useful in the present discussion to highlight the difference between $C_{h, g}^{(n)}$ and well-known covariances associated with correlation functions.

The covariances appearing in a similar construction for the generating functions of correlations are related, rather, to an autonomous difference equation on the torus $\tilde{\mathbb{T}}_{n} \doteq \mathbb{T}_{n} \cap(-\beta$, $\beta$, which refers to the case $\alpha \in \mathbb{T}_{n} \cap(0, \beta]$ in the right-hand side of (9). This is related to the case $k<n$ in Corollary 4.8. In this situation, as is done for instance in [BGPS94, Section 3], [GM10, Section 3.2], [GMP16, Section 5.1.], large determinants (or Pfaffians) of the covariances can be dealt with by using a combination of multiscale analyses for the so-called Matsubara UV problem together with the usual Gram bound for determinants. It is precisely what is done in [GLM02, Section 7] for weakly interacting fermions on a lattice: They consider the sequence $\left\{\mathrm{E}_{l}^{\Psi}=\mathrm{D}_{l}\right\}_{l \in \mathbb{R}^{+}}$of particle density observables (139) and expand the exponential $\mathrm{e}^{s \mathrm{E}_{l}^{\Psi}}=\mathrm{e}^{s \mathrm{D}_{l}}$ in (7) to compute the resulting series of correlation functions via constructive methods. The choice $\mathrm{E}_{l}^{\Psi}=\mathrm{D}_{l}$ strongly simplifies the combinatorics because $\mathrm{D}_{l}, l \in \mathbb{R}^{+}$, are projection operators. A generalization of this approach to any thermodynamic sequence $\left\{\mathrm{E}_{l}^{\Psi}\right\}_{l \in \mathbb{R}^{+}}$is not clear.

Meanwhile, applying multiscale decompositions in the Matsubara frequency together with the usual Gram bound for covariances like $C_{h, 0}^{(n)}$ would not directly work, because the operators $\mathfrak{u}_{h}$ and $\partial$ in (10) do not commute, in contrast to the autonomous case. Last but not least, these studies using multiscale methods require Bogoliubov transformations to recover the gauge invariant case $(g=0)$.

However, [dSPS08] showed that such multiscale analyses to tackle the Matsubara UV problem are not necessary, at least in the autonomous case, by proving a new bound for determinants that generalizes the original Gram bound, see [dSPS08, Theorem 1.3]. In the same spirit, we recently derived [BdSP17b] bounds that also do not need the UV regularization of the Matsubara frequency, with the technical advantage that the given covariance does not need to be decomposed as in [dSPS08, Eq. (8)] to obtain determinant bounds. Moreover, the estimate in [BdSP17b] is sharp (or optimal) and holds true for all (even unbounded cases, not being limited to semibounded) one-particle Hamiltonians. It is important to stress that our approach to Pfaffian (or determinant) bounds does not preclude the need of multiscale analysis to handle IR singularities of covariances, as it is done, for instance, in [GM10, GMP16]. Note however that a positive temperature or the presence of a gap (at zero energy) in the dispersion relation of free particles regularizes such IR singularities and our method makes any multiscale analysis superfluous, at least for a small enough interaction strength, in these two cases.

As remarked in [BdSP17b, Section 1.4], using multiscale decomposition to address the Matsubara UV problem can render the analysis less transparent and avoiding this kind of procedure brings various technical benefits: In the finite-dimensional case, we extend the proof of [BdSP17b, Corollary 1.4] to bound large Pfaffians of fermionic covariances $C_{h, g}^{(n)}$ in the non-autonomous cases, allowing us in a futur work [BdSPX] to directly compute, at weak coupling, the logarithmic moment generating function (8) via constructive methods. In fact, if the free Hamiltonian is generic, then our Pfaffians bounds yield the convergence of usual expansions (in particular the Brydges-Kennedy expansion) for the generating function, whenever the interaction strength is small as compared to $\beta^{-(d+1)}$. If the 
spectrum of the one-particle free Hamiltonian has positive distance $\delta$ to zero (i.e., the Hamiltonian has a spectral gap of size $\delta>0$ ) then the convergence radius is of order $\delta^{d+1}$, uniformly with respect to the temperature. In particular, the limit $\beta \rightarrow \infty$ can be taken and large deviation results can be proven for ground states, in this case. Note that we represent the logarithmic moment generating function of large deviation theory in a way that is formally the same as the usual one for the pressure of fermion systems, while keeping good Pfaffian (or determinant) bounds. Thus, very likely, also the gapless case can be handled at zero temperature, by combining our results with (a suitable version of) usual multiscale decompositions to tame the IR singularity of covariances at small energies.

In [BdSP17b], constructions involving quasi-free states in suitably chosen CAR algebras are used, whereas in the present case Grassmann-algebra computations turn out to be more efficient by allowing us to represent determinants as traces. Like in [BdSP17b, Section 1.6], Hölder inequalities for noncommutative $L^{p}$-spaces, here for Schatten norms (see (84)-(86)), are pivotal tools and replace the celebrated Gram bounds on which all previous results, like [BGPS94, dSPS08, GLM02, GM10, GMP16], are explicitly based.

\section{Remark 1.2}

Since our methods do not need translation invariance, our results are also useful for handling usual correlation functions as well as logarithmic moment generating functions, for fermionic systems with random, but ergodic, interactions, as defined for instance in [BdSP15].

To conclude, the main results are Theorem 4.6, 5.11 and 5.13 as well as Corollaries 4.8, 5.2 and 5.3. Additionally, the mathematical methods used here are developed in a systematic and general way. Some of them are, to some extent, well-known, like for instance the trace formula (Theorem 4.1). Other ingredients are less standard, like the definition of the "circle" product (Definition 3.9) and the Chernoff product formula (Definition 4.2, Theorem 4.3), both introduced in the Ph.D. thesis [dSP05]. However, the extension of this technology to general self-dual CAR algebra is new. In fact, this paper will serve as a reference for further research works.

The paper is organized as follows:

- Section 2 presents self-dual CAR algebra as well as the corresponding quasi-free dynamics and states. It sets up the primary mathematical framework used in the present study.

- Section 3 establishes a relation between self-dual CAR algebra and Grassmann (or exterior) algebra. By using the Berezin integral, we define in particular a family of "circle" products, indexed by so-called basis projections, converting any Grassmann algebra into a family of selfdual CAR algebras.

- In Section 4, we derive a Feynman-Kac-like formula for logarithmic moment generating functions by using Gaussian Berezin integrals and an appropriately chosen basis projection.

- Section 5 studies so-called Pfaffian bounds of the fermionic covariances of the Gaussian Berezin integrals that represent logarithmic moment generating functions as well as their expression as functions of two-point correlation functions. The summability of the covariance is also proven here for very general fermion systems on the lattice.

- CAR algebra is more widely known than its self-dual counterpart. We thus provide in Section 6 a pedagogical introduction to CAR algebra in relation to self-dual CAR algebra. For the reader's convenience, we also add a concise explanation of the Fock representation of CAR algebra. 
- Finally, we describe in Sections 7 and 8 the overall conceptual framework beyond the concrete technical problems tackled here in order to highlight our results. This also makes the discussions in the introduction more precise. In particular, in Section 7 we mathematically define the large deviations (LD) formalism loosely discussed above, while Section 8 explains its applications to quantum spin systems or fermions on lattices.

\section{Notation 1.3}

(i) A norm on a generic vector space $\mathcal{X}$ is denoted by $\|\cdot\|_{\mathcal{X}}$ and the identity map of $\mathcal{X}$ by $\mathbf{1}_{\mathcal{X}}$. The space of all bounded linear operators on $\left(\mathcal{X},\|\cdot\|_{\mathcal{X}}\right)$ is denoted by $\mathcal{B}(\mathcal{X})$. The unit element of any algebra $\mathcal{X}$ is denoted by 1 , provided it exists. The scalar product of any Hilbert space $\mathcal{X}$ is denoted by $\langle\cdot, \cdot\rangle_{\mathcal{X}}$ and $\operatorname{Tr}_{\mathcal{X}}$ represents the usual trace on $\mathcal{B}(\mathcal{X})$.

(ii) For each $k \in \mathbb{N}_{0}$, denote by $\mathcal{X}^{(k)}$ a copy of some vector space $\mathcal{X}$. The corresponding copy of $\xi \in \mathcal{X}$ is denoted by $\xi^{(k)}$.

(iii) We denote by $D$ any positive and finite generic constant. These constants do not need to be the same from one statement to another.

\section{Self-dual CAR Algebra and Bilinear Hamiltonians}

\subsection{Self-dual CAR Algebra}

If not otherwise stated, $\mathcal{H}$ always stands for a finite-dimensional (complex) Hilbert space with even dimension $\operatorname{dim} \mathcal{H} \in 2 \mathbb{N}$. Let $\mathfrak{A}$ be an antiunitary involution on $\mathcal{H}$, i.e., an antilinear map from $\mathcal{H}$ to $\mathcal{H}$ such that $\mathfrak{A}^{2}=\mathbf{1}_{\mathcal{H}}$ and

$$
\left\langle\mathfrak{A} \varphi_{1}, \mathfrak{A} \varphi_{2}\right\rangle_{\mathcal{H}}=\left\langle\varphi_{2}, \varphi_{1}\right\rangle_{\mathcal{H}}, \quad \varphi_{1}, \varphi_{2} \in \mathcal{H}
$$

The space $\mathcal{H}$ endowed with the involution $\mathfrak{A}$ is named a self-dual Hilbert space and yields self-dual $C A R^{10}$ algebra, which is defined as follows:

\section{Definition 2.1 (Self-dual CAR algebra)}

A self-dual CAR algebra $\operatorname{sCAR}(\mathcal{H}, \mathfrak{A}) \equiv(\operatorname{sCAR}(\mathcal{H}, \mathfrak{A}),+, \cdot, *)$ is a $C^{*}$-algebra generated by a unit 1 and a family $\{\mathrm{B}(\varphi)\}_{\varphi \in \mathcal{H}}$ of elements satisfying Conditions (a)-(c):

(a) The map $\varphi \mapsto \mathrm{B}(\varphi)^{*}$ is (complex) linear.

(b) $\mathrm{B}(\varphi)^{*}=\mathrm{B}(\mathfrak{A}(\varphi))$ for any $\varphi \in \mathcal{H}$.

(c) The family $\{\mathrm{B}(\varphi)\}_{\varphi \in \mathcal{H}}$ satisfies the CAR: For any $\varphi_{1}, \varphi_{2} \in \mathcal{H}$,

$$
\mathrm{B}\left(\varphi_{1}\right) \mathrm{B}\left(\varphi_{2}\right)^{*}+\mathrm{B}\left(\varphi_{2}\right)^{*} \mathrm{~B}\left(\varphi_{1}\right)=\left\langle\varphi_{1}, \varphi_{2}\right\rangle_{\mathcal{H}} \mathbf{l}
$$

To the best of our knowledge, this notion was introduced by Araki in [A68]. He changed his definition two years later in [A70] by replacing the linearity of the map $\varphi \mapsto \mathrm{B}(\varphi)^{*}$ with its antilinearity. We maintain here the original definition from [A68], and explicitly add the fact that $\operatorname{sCAR}(\mathcal{H}, \mathfrak{A})$ is not only a $*$-algebra but also a $C^{*}$-algebra (see, e.g., [A68, Lemma 4.5]).

\section{Remark 2.2}

By the CAR (11), the antilinear map $\varphi \mapsto \mathrm{B}(\varphi)$ is necessarily injective and contractive. Therefore, $\mathcal{H}$ can be imbedded in $\operatorname{sCAR}(\mathcal{H}, \mathfrak{A})$.

\footnotetext{
${ }^{10} \mathrm{CAR}$ refers to canonical anti-commutation relations.
} 


\section{Remark 2.3}

Self-dual CAR algebras are also well-defined even if the Hilbert space $\mathcal{H}$ has infinite or odd dimension. In the odd case, they are *-isomorphic to the direct product of a CAR algebra and a twodimensional abelian algebra, see [A68, Lemmata 3.3, 3.7]. This situation is, however, not important in our study. The infinite-dimension case only shows up in Section 5.3, when we consider $\mathcal{H}_{\infty}$. See again [A68, Lemma 3.3].

Strictly speaking, Conditions (a)-(c) of Definition 2.1 only define self-dual CAR algebras up to Bogoliubov $*$-automorphisms ${ }^{11}$ (see (15)). In Section 3 we construct explicit $*$-isomorphic self-dual CAR algebras from $\mathcal{H}$ and $\mathfrak{A}$. This is done via the following concept of basis projection [A68, Definition 3.5], which also highlights the relationship between CAR algebras and their self-dual counterparts.

\section{Definition 2.4 (Basis projections)}

A basis projection associated with $(\mathcal{H}, \mathfrak{A})$ is an orthogonal projection $P \in \mathcal{B}(\mathcal{H})$ satisfying $\mathfrak{A} P \mathfrak{A}=$ $P^{\perp} \doteq \mathbf{1}_{\mathcal{H}}-P$. We denote by $\mathfrak{h}_{P}$ the range $\operatorname{ran} P$ of the basis projection $P$.

Note that $\mathfrak{h}_{P}$ must satisfy the conditions

$$
\mathfrak{A}\left(\mathfrak{h}_{P}\right)=\mathfrak{h}_{P}^{\perp} \quad \text { and } \quad \mathfrak{A}\left(\mathfrak{h}_{P}^{\perp}\right)=\mathfrak{h}_{P}
$$

for any basis projection $P$.

By [A68, Lemma 3.3], an explicit basis projection $P \in \mathcal{B}(\mathcal{H})$ associated with $(\mathcal{H}, \mathfrak{A})$ can always be constructed because $\operatorname{dim} \mathcal{H} \in 2 \mathbb{N}$. Moreover, $\varphi \mapsto(\mathfrak{A} \varphi)^{*}$ is a unitary map from $\mathfrak{h} \frac{\perp}{P}$ to the dual space $\mathfrak{h}_{P}^{*}$. In this case we can identify $\mathcal{H}$ with

$$
\mathcal{H} \equiv \mathfrak{h}_{P} \oplus \mathfrak{h}_{P}^{*}
$$

and

$$
\mathrm{B}(\varphi) \equiv \mathrm{B}(P \varphi)+\mathrm{B}\left(\mathfrak{A} P^{\perp} \varphi\right)^{*} .
$$

Therefore, there is a natural isomorphism of $C^{*}$-algebras from $\operatorname{sCAR}(\mathcal{H}, \mathfrak{A})$ to the CAR algebra $\operatorname{CAR}\left(\mathfrak{h}_{P}\right)$ generated by the unit 1 and $\left\{\mathrm{B}_{P}(\varphi)\right\}_{\varphi \in \mathfrak{h}_{P}}$. See Definition 6.1 and also [A68, Lemma 3.3] for more details. In other words, a basis projection $P$ can be used to fix so-called annihilation and creations operators.

Sections 6.1-6.2 provide a pedagogical construction of self-dual CAR algebra starting from a CAR algebra $\operatorname{CAR}(\mathfrak{h})$ associated with some one-particle Hilbert space $\mathfrak{h}$, as explained in Definition 6.1. CAR algebra is more widely known than its self-dual analogue. However, the notion of self-dual CAR algebra is more flexible because the one-particle Hilbert space is not fixed anymore. In fact, for each basis projection $P$ associated with $(\mathcal{H}, \mathfrak{A})$, by $(13), \mathfrak{h}_{P}$ can be seen as a one-particle Hilbert space. As shown in [A68, A70], this approach to CAR naturally arises in the diagonalization of quadratic fermionic Hamiltonians (Definition 2.5), via Bogoliubov transformations defined as follows:

For any unitary operator $U \in \mathcal{B}(\mathcal{H})$ such that $U \mathfrak{A}=\mathfrak{A} U$, the family of elements $\mathrm{B}(U \varphi)_{\varphi \in \mathcal{H}}$ satisfies Conditions (a)-(c) of Definition 2.1 and, together with the unit 1 , generates $\operatorname{sCAR}(\mathcal{H}, \mathfrak{A})$. Like in [A70, Section 2], such a unitary operator $U \in \mathcal{B}(\mathcal{H})$ commuting with the antiunitary map $\mathfrak{A}$ is named a Bogoliubov transformation, and the unique $*$-automorphism $\chi_{U}$ such that

$$
\chi_{U}(\mathrm{~B}(\varphi))=\mathrm{B}(U \varphi), \quad \varphi \in \mathcal{H},
$$

is called in this case a Bogoliubov $*$-automorphism. Note that a Bogoliubov transformation $U \in \mathcal{B}(\mathcal{H})$ always satisfies

$$
\operatorname{det}(U)=\operatorname{det}(\mathfrak{A} U \mathfrak{A})=\overline{\operatorname{det}(U)}= \pm 1 \text {. }
$$

\footnotetext{
${ }^{11}$ An analogous result for CAR algebra is, for instance, given by [BR96b, Theorem 5.2.5].
} 
(Recall that, by definition, a Bogoliubov transformation is unitary.) If $\operatorname{det}(U)=1$, we say that $U$ has positive orientation. See Lemma 3.4. Otherwise $U$ is said to have negative orientation. These properties are also called even and odd [EK98, Chap. 6].

Clearly, if $P$ is a basis projection associated with $(\mathcal{H}, \mathfrak{A})$ and $U \in \mathcal{B}(\mathcal{H})$ a Bogoliubov transformation, then $P_{U} \doteq U P U^{*}$ is another basis projection. Conversely, for any pair of basis projections $P_{1}, P_{2} \in \mathcal{B}(\mathcal{H})$ there is a (generally not unique) Bogoliubov transformation $U$ such that $P_{2}=U P_{1} U^{*}$. See [A68, Lemma 3.6]. In particular, Bogoliubov transformations map one-particle Hilbert spaces onto one another.

In Section 3, we show that self-dual CAR algebra naturally arises in the framework of Grassmann algebra. Self-dual CAR algebra also allows us to elegantly treat non-gauge invariant models. That is why we focus on $\operatorname{sCAR}(\mathcal{H}, \mathfrak{A})$ and employ the terminology $\operatorname{CAR}(\mathfrak{h})$ of (common) CAR algebra only to pedagogical discussions.

\subsection{Bilinear Elements of Self-dual CAR Algebra}

An element $A \in \operatorname{sCAR}(\mathcal{H}, \mathfrak{A})$, satisfying $\chi_{-\mathbf{1}_{\mathcal{H}}}(A)=A$ or $\chi_{-\mathbf{1}_{\mathcal{H}}}(A)=-A$ (see (15)), is called, respectively, even or $o d d$. The subspace of even elements is a sub- $C^{*}$-algebra of $\operatorname{sCAR}(\mathcal{H}, \mathfrak{A})$.

An important class of even elements in quantum physics is provided by quadratic fermionic Hamiltonians. These refer to the self-adjoint (even) elements of the CAR algebra which are quadratic in the creation and annihilation operators, like for instance the Bogoliubov approximation of the celebrated (reduced) BCS model. In the context of self-dual CAR algebra, those elements are called bilinear Hamiltonians by Araki [A68, Lemma 4.4, Definition 4.7] and are self-adjoint bilinear elements:

\section{Definition 2.5 (Bilinear elements of self-dual CAR algebra)}

Given an orthonormal basis $\left\{\psi_{i}\right\}_{i \in I}$ of $\mathcal{H}$, we define the bilinear element associated with $H \in \mathcal{B}(\mathcal{H})$ to be

$$
\langle\mathrm{B}, H \mathrm{~B}\rangle \doteq \sum_{i, j \in I}\left\langle\psi_{i}, H \psi_{j}\right\rangle_{\mathcal{H}} \mathrm{B}\left(\psi_{j}\right) \mathrm{B}\left(\psi_{i}\right)^{*}
$$

Note that $\langle\mathrm{B}, H \mathrm{~B}\rangle$ does not depend on the particular choice of the orthonormal basis, but does depend on the choice of generators $\{\mathrm{B}(\varphi)\}_{\varphi \in \mathcal{H}}$ of the self-dual CAR algebra $\operatorname{sCAR}(\mathcal{H}, \mathfrak{A})$. Moreover,

$$
\begin{aligned}
\langle\mathrm{B}, H \mathrm{~B}\rangle & =\sum_{i, j \in I}\left\langle\mathfrak{A} \psi_{i}, H \psi_{j}\right\rangle_{\mathcal{H}} \mathrm{B}\left(\psi_{j}\right) \mathrm{B}\left(\psi_{i}\right)=\sum_{i, j \in I}\left\langle\psi_{i}, H \mathfrak{A} \psi_{j}\right\rangle_{\mathcal{H}} \mathrm{B}\left(\psi_{j}\right)^{*} \mathrm{~B}\left(\psi_{i}\right)^{*} \\
& =\sum_{i, j \in I}\left\langle\psi_{j}, \mathfrak{A} H^{*} \mathfrak{A} \psi_{i}\right\rangle_{\mathcal{H}} \mathrm{B}\left(\psi_{j}\right)^{*} \mathrm{~B}\left(\psi_{i}\right)
\end{aligned}
$$

for all $H \in \mathcal{B}(\mathcal{H})$, and by $(11)$, bilinear elements of $\operatorname{sCAR}(\mathcal{H}, \mathfrak{A})$ have adjoints equal to

$$
\langle\mathrm{B}, H \mathrm{~B}\rangle^{*}=\left\langle\mathrm{B}, H^{*} \mathrm{~B}\right\rangle, \quad H \in \mathcal{B}(\mathcal{H}) .
$$

See, e.g., [A70, Eqs. (7.5)-(7.6)].

Bilinear Hamiltonians are then defined as bilinear elements associated with self-adjoint operators $H=H^{*} \in \mathcal{B}(\mathcal{H})$. They include all second quantizations of one-particle Hamiltonians (see Equations (4) and (120)), but also models that are not gauge invariant (see Equations (5) and (121)). Important models in condensed matter physics, like in the BCS theory of superconductivity, are bilinear Hamiltonians that are not gauge invariant.

To study all bilinear elements of self-dual CAR algebra, one can reduce the analysis of bilinear elements to a special class of operators $H$ : 


\section{Lemma 2.6 (The set of bilinear elements of self-dual CAR algebra)}

Given a finite-dimensional self-dual CAR algebra $\operatorname{SCAR}(\mathcal{H}, \mathfrak{A})$,

$$
\left\{\langle\mathrm{B}, H \mathrm{~B}\rangle+\lambda_{\mathbf{l}}: \lambda \in \mathbb{C}, H \in \mathcal{B}(\mathcal{H})\right\}=\left\{\langle\mathrm{B}, H \mathrm{~B}\rangle+\lambda_{\mathbf{l}}: \lambda \in \mathbb{C}, H \in \mathcal{B}(\mathcal{H}), H^{*}=-\mathfrak{A} H \mathfrak{A}\right\}
$$

Proof. Observe that, for any $H \in \mathcal{B}(\mathcal{H})$,

$$
\langle\mathrm{B}, H \mathrm{~B}\rangle+\left\langle\mathrm{B}, \mathfrak{A} H^{*} \mathfrak{A B}\right\rangle=\operatorname{Tr}_{\mathcal{H}}(H) \perp
$$

(cf. [A70, Eq. (7.7)]). This implies that, for any $H \in \mathcal{B}(\mathcal{H})$,

$$
\langle\mathrm{B}, H \mathrm{~B}\rangle=\langle\mathrm{B}, \mathbf{K}(H) \mathrm{B}\rangle+\frac{1}{2} \operatorname{Tr}_{\mathcal{H}}(H) \mathbf{\perp}
$$

with $\mathbf{K}$ being the linear map from $\mathcal{B}(\mathcal{H})$ to itself defined by

$$
\mathbf{K}(H) \doteq \frac{1}{2}\left(H-\mathfrak{A} H^{*} \mathfrak{A}\right), \quad H \in \mathcal{B}(\mathcal{H})
$$

(This map already appears in [A68, Eqs. (4.16), (5.16)].) Since, for any $H \in \mathcal{B}(\mathcal{H})$,

$$
\mathbf{K}(H)^{*}=\mathbf{K}\left(H^{*}\right)=-\mathfrak{A} \mathbf{K}(H) \mathfrak{A},
$$

the second equality of the lemma results from (20).

Therefore, by Lemma 2.6, all of our analysis of bilinear elements can be restricted, without loss of generality, to operators $H \in \mathcal{B}(\mathcal{H})$ satisfying $H^{*}=-\mathfrak{A} H \mathfrak{A}$. We call such operators self-dual operators:

\section{Definition 2.7 (Self-dual operators)}

A self-dual operator on $(\mathcal{H}, \mathfrak{A})$ is an operator $H \in \mathcal{B}(\mathcal{H})$ satisfying the equality $H^{*}=-\mathfrak{A} H \mathfrak{A}$. If, additionally, $H$ is self-adjoint, then we say that it is a self-dual Hamiltonian on $(\mathcal{H}, \mathfrak{A})$.

Note that a self-dual operator $H$ has zero trace, i.e.,

$$
\operatorname{Tr}_{\mathcal{H}}(H)=\langle\mathrm{B}, H \mathrm{~B}\rangle+\left\langle\mathrm{B}, \mathfrak{A} H^{*} \mathfrak{A B}\right\rangle=0
$$

by Equation (19).

We say that the basis projection $P$ (Definition 2.4) (block-) "diagonalizes" the self-dual operator $H \in \mathcal{B}(\mathcal{H})$ whenever

$$
H=\frac{1}{2}\left(P H_{P} P-P^{\perp} \mathfrak{A} H_{P}^{*} \mathfrak{A} P^{\perp}\right), \quad \text { with } \quad H_{P} \doteq 2 P H P \in \mathcal{B}\left(\mathfrak{h}_{P}\right) .
$$

In this situation, we also say that the basis projection $P$ diagonalizes $\langle\mathrm{B}, H \mathrm{~B}\rangle$, similarly to [A68, Definition 5.1]: By Definition 2.1 and Equation (23), for any orthonormal basis $\left\{\psi_{j}\right\}_{j \in J}$ of $\mathfrak{h}_{P}$,

$$
\langle\mathrm{B}, H \mathrm{~B}\rangle=2 \sum_{i, j \in J}\left\langle\psi_{i}, H \psi_{j}\right\rangle_{\mathcal{H}} \mathrm{B}\left(\psi_{j}\right) \mathrm{B}\left(\psi_{i}\right)^{*}+\operatorname{Tr}_{\mathcal{H}}\left(P^{\perp} H P^{\perp}\right) \perp
$$

Compare this equation with (118)-(119) and (120).

By the spectral theorem, for any self-dual Hamiltonian $H$ on $(\mathcal{H}, \mathfrak{A})$, there is always a basis projection $P$ diagonalizing $H$. In quantum physics, as discussed in Section $2.1, \mathfrak{h}_{P}$ is in this case the one-particle Hilbert space and $H_{P}$ the one-particle Hamiltonian. In particular, the first term of the right-hand side of (24) is the so-called second quantization of $H_{P}$. 


\subsection{Quasi-Free Dynamics and States}

Bilinear Hamiltonians are used to define so-called quasi-free dynamics: For any $H=H^{*} \in \mathcal{B}(\mathcal{H})$, we define the continuous group $\left\{\tau_{t}\right\}_{t \in \mathbb{R}}$ of $*$-automorphisms of $\operatorname{sCAR}(\mathcal{H}, \mathfrak{A})$ by

$$
\tau_{t}(A) \doteq \mathrm{e}^{-i t\langle\mathrm{~B}, H \mathrm{~B}\rangle} A \mathrm{e}^{i t\langle\mathrm{~B}, H \mathrm{~B}\rangle}, \quad A \in \operatorname{sCAR}(\mathcal{H}, \mathfrak{A}), t \in \mathbb{R}
$$

(see Definition 2.5 and Equation (19). Provided $H$ is a self-dual Hamiltonian on $(\mathcal{H}, \mathfrak{A})$ (Definition 2.7), this group is a quasi-free dynamics, that is, a continuous group of Bogoliubov $*$-automorphisms, as defined in Equation (15). This can be seen from the following assertion:

\section{Lemma 2.8 (From bilinear Hamiltonians to quasi-free dynamics)}

Take any self-dual operator $H$ on $(\mathcal{H}, \mathfrak{A})$ (Definition 2.7). Then, for all $z \in \mathbb{C}$ and $\varphi \in \mathcal{H}$,

$$
\exp \left(-\frac{z}{2}\langle\mathrm{~B}, H \mathrm{~B}\rangle\right) \mathrm{B}(\varphi)^{*} \exp \left(\frac{z}{2}\langle\mathrm{~B}, H \mathrm{~B}\rangle\right)=\mathrm{B}\left(\mathrm{e}^{z H} \varphi\right)^{*} .
$$

Proof. The proof is completely standard. We outline it for completeness. As usual, $\left[B_{1}, B_{2}\right] \doteq$ $B_{1} B_{2}-B_{2} B_{1}$ is the commutator of two elements $B_{1}, B_{2} \in \operatorname{sCAR}(\mathcal{H}, \mathfrak{A})$.

Fix $H \in \mathcal{B}(\mathcal{H})$ and $\varphi \in \mathcal{H}$. Straightforward computations using Definitions 2.1 and 2.5, together with the properties of the antiunitary involution $\mathfrak{A}$, lead to the equality

$$
\frac{1}{2}\left[\langle\mathrm{~B}, H \mathrm{~B}\rangle, \mathrm{B}(\varphi)^{*}\right]=-\mathrm{B}(\mathbf{K}(H) \varphi)^{*},
$$

where $\mathbf{K}$ is the linear map on $\mathcal{B}(\mathcal{H})$ defined by (21). This statement also refers to [A68, Eqs. (4.15)(4.16)]. In particular, if $H^{*}=-\mathfrak{A} H \mathfrak{A}$ (cf. Definition 2.7), then $\mathbf{K}(H)=H$ and, by (25), it follows that, for any $z \in \mathbb{C}$,

$$
\partial_{z}\left\{\exp \left(\frac{z}{2}\langle\mathrm{~B}, H \mathrm{~B}\rangle\right) \mathrm{B}\left(\mathrm{e}^{z H} \varphi\right)^{*} \exp \left(-\frac{z}{2}\langle\mathrm{~B}, H \mathrm{~B}\rangle\right)\right\}=0
$$

which proves the lemma.

Bilinear Hamiltonians are also used to define quasi-free states. States are positive and normalized linear functionals $\rho \in \operatorname{sCAR}(\mathcal{H}, \mathfrak{A})^{*}$, i.e., $\rho(\mathbf{l})=1$ and $\rho\left(A^{*} A\right) \geq 0$ for all $A \in \operatorname{sCAR}(\mathcal{H}, \mathfrak{A})$. They are said to be quasi-free when, for all $N \in \mathbb{N}_{0}$ and $\varphi_{0}, \ldots, \varphi_{2 N} \in \mathcal{H}$,

$$
\rho\left(\mathrm{B}\left(\varphi_{0}\right) \cdots \mathrm{B}\left(\varphi_{2 N}\right)\right)=0
$$

while, for all $N \in \mathbb{N}$ and $\varphi_{1}, \ldots, \varphi_{2 N} \in \mathcal{H}$,

$$
\rho\left(\mathrm{B}\left(\varphi_{1}\right) \cdots \mathrm{B}\left(\varphi_{2 N}\right)\right)=\operatorname{Pf}\left[\rho\left(\mathbb{O}_{k, l}\left(\mathrm{~B}\left(\varphi_{k}\right), \mathrm{B}\left(\varphi_{l}\right)\right)\right)\right]_{k, l=1}^{2 N},
$$

where

$$
\mathbb{O}_{k, l}\left(A_{1}, A_{2}\right) \doteq\left\{\begin{array}{cc}
A_{1} A_{2} & \text { for } \quad k<l \\
-A_{2} A_{1} & \text { for } k>l \\
0 & \text { for } k=l
\end{array}\right.
$$

In Equation (27), Pf is the usual Pfaffian defined by

$$
\operatorname{Pf}\left[M_{k, l}\right]_{k, l=1}^{2 N} \doteq \frac{1}{2^{N} N !} \sum_{\pi \in \mathcal{S}_{2 N}}(-1)^{\pi} \prod_{j=1}^{N} M_{\pi(2 j-1), \pi(2 j)}
$$

for any $2 N \times 2 N$ skew-symmetric matrix $M \in \operatorname{Mat}(2 N, \mathbb{C})$. Note that (27) is equivalent to the definition given either in [A70, Definition 3.1] or in [EK98, Equation (6.6.9)]. 
Quasi-free states are therefore particular states that are uniquely defined by two-point correlation functions, via (26)-(27). In fact, a quasi-free state $\rho$ is uniquely defined by its so-called symbol, that is, a positive operator $S_{\rho} \in \mathcal{B}(\mathcal{H})$ such that

$$
0 \leq S_{\rho} \leq \mathbf{1}_{\mathcal{H}} \quad \text { and } \quad S_{\rho}+\mathfrak{A} S_{\rho} \mathfrak{A}=\mathbf{1}_{\mathcal{H}}
$$

through the conditions

$$
\left\langle\varphi_{1}, S_{\rho} \varphi_{2}\right\rangle_{\mathcal{H}}=\rho\left(\mathrm{B}\left(\varphi_{1}\right) \mathrm{B}\left(\mathfrak{A} \varphi_{2}\right)\right), \quad \varphi_{1}, \varphi_{2} \in \mathcal{H}
$$

For more details on symbols of quasi-free states, see [A70, Lemma 3.2 ${ }^{12}$. Conversely, any selfadjoint operator satisfying (29) uniquely defines a quasi-free state through Equation (30). See [A70, Lemma 3.3]. In physics, $S_{\rho}$ is called the one-particle density matrix of the system.

Quasi-free states obviously depend on the choice of generators of the self-dual CAR algebra. An example of a quasi-free state is provided by the tracial state:

\section{Definition 2.9 (Tracial state)}

The tracial state $\operatorname{tr} \in \operatorname{sCAR}(\mathcal{H}, \mathfrak{A})^{*}$ is the quasi-free state with symbol $S_{\mathrm{tr}} \doteq \frac{1}{2} \mathbf{1}_{\mathcal{H}}$.

This is the usual tracial state known for CAR algebra, as one can see from Remark 6.4. It can be used to highlight the relationship between quasi-free states and bilinear Hamiltonians:

\section{Lemma 2.10 (From Bilinear Hamiltonians to quasi-free states)}

Take $\beta \in(0, \infty)$ and any self-dual Hamiltonian $H$ on $(\mathcal{H}, \mathfrak{A})$ (Definition 2.7). Then, the positive operator $\left(1+\mathrm{e}^{-\beta H}\right)^{-1}$ satisfies Condition (29) and is the symbol of a quasi-free state $\rho_{H}$ satisfying

$$
\rho_{H}(A)=\frac{\operatorname{tr}\left(A \exp \left(\frac{\beta}{2}\langle\mathrm{~B}, H \mathrm{~B}\rangle\right)\right)}{\operatorname{tr}\left(\exp \left(\frac{\beta}{2}\langle\mathrm{~B}, H \mathrm{~B}\rangle\right)\right)}, \quad A \in \operatorname{sCAR}(\mathcal{H}, \mathfrak{A}) .
$$

Proof. Let $H \in \mathcal{B}(\mathcal{H})$ be such that $H=H^{*}=-\mathfrak{A} H \mathfrak{A}$. Then, by using a Taylor series, one verifies that the map

$$
\beta \mapsto f(\beta) \doteq \mathfrak{A}(1+\exp (\beta H))^{-1} \mathfrak{A}-(1+\exp (\beta \mathfrak{A} H \mathfrak{A}))^{-1}
$$

vanishes for small enough $\beta \in \mathbb{R}$ and, since this function is real analytic on the whole real line $\mathbb{R}$, it can only be the zero function on $\mathbb{R}$. Using elementary computations, it follows that $S=\left(1+\mathrm{e}^{-\beta H}\right)^{-1}$ satisfies (29) for any $\beta \in \mathbb{R}$.

Note that the denominator of (31) is strictly positive because the tracial state tr is faithful. Equation (31) follows by observing that the states of both sides of this equality are the unique KMS state of the same quasi-free dynamics (cf. Lemma 2.8 and [A70, Theorem 3]). We omit the details and refer to [A70, Corollary 6.3] for the complete proof.

The state $\rho_{H}$ is named the Gibbs state, or thermal equilibrium state, associated with the self-dual (one-particle) Hamiltonian $H$ on $(\mathcal{H}, \mathfrak{A})$ at fixed $\beta \in(0, \infty)$. The parameter $\beta \in(0, \infty)$ is physically interpreted as being the inverse temperature of the fermion system.

\section{Remark 2.11}

By Definition 2.4, any basis projection associated with $(\mathcal{H}, \mathfrak{A})$ can be seen as a symbol of a quasifree state on $\operatorname{sCAR}(\mathcal{H}, \mathfrak{A})$. Such state is pure and called a Fock state [A70, Lemma 4.3]. Araki shows in [A70, Lemmata 4.5-4.6] that any quasi-free state can be seen as the restriction of a quasifree state on $\operatorname{sCAR}(\mathcal{H} \oplus \mathcal{H}, \mathfrak{A} \oplus(-\mathfrak{A}))$, the symbol of which is a basis projection associated with $(\mathcal{H} \oplus \mathcal{H}, \mathfrak{A} \oplus(-\mathfrak{A}))$. This procedure is called purification of the quasi-free state.

\footnotetext{
${ }^{12}$ As already remarked above, [A70, Lemma 3.2] defines the map $\varphi \mapsto \mathrm{B}(\varphi)$ to be linear and the corresponding definition of symbols changes accordingly.
} 
The important issue of the present study is to show that so-called Brydges-Kennedy tree expansions allow a construction of the logarithmic moment generating function (7) of Gibbs states associated with Hamiltonians of the form (3), that is,

$$
\mathbf{H}=-\frac{1}{2}\langle\mathrm{~B}, H \mathrm{~B}\rangle+W, \quad W=W^{*} \in \operatorname{sCAR}(\mathcal{H}, \mathfrak{A})^{*}
$$

with $H \in \mathcal{B}(\mathcal{H})$ such that $H=H^{*}=-\mathfrak{A} H \mathfrak{A}$, provided $W$ is sufficiently small in an appropriate sense.

\section{Realization of Self-dual CAR Algebra as a Grassmann Algebra}

\subsection{Grassmann Algebra}

Grassmann algebra, also called exterior algebra, is a well-known mathematical structure, see, e.g., [Di73, Appendix], or [FKT02] for an exposition in the context of QFT. For completeness and to fix notation, we outline its construction:

(i): Let $\mathcal{X}$ be a topological vector space. Then, $\mathcal{X}^{*}$ denotes, as is usual, the space of all continuous linear functionals on $\mathcal{X}$. For every $n \in \mathbb{N}$ and $x_{1}^{*}, \ldots, x_{n}^{*} \in \mathcal{X}^{*}$, we define the completely antisymmetric $n$-linear form $x_{1}^{*} \wedge \cdots \wedge x_{n}^{*}$ from $\mathcal{X}^{n}$ to $\mathbb{C}$ by

$$
x_{1}^{*} \wedge \cdots \wedge x_{n}^{*}\left(y_{1}, \ldots, y_{n}\right) \doteq \operatorname{det}\left(\left(x_{k}^{*}\left(y_{l}\right)\right)_{k, l=1}^{n}\right), \quad y_{1}, \ldots, y_{n} \in \mathcal{X} .
$$

In particular, for any permutation $\pi$ of $n \in \mathbb{N}$ elements with sign $(-1)^{\pi}$,

$$
x_{1}^{*} \wedge \cdots \wedge x_{n}^{*} \doteq(-1)^{\pi} x_{\pi(1)}^{*} \wedge \cdots \wedge x_{\pi(n)}^{*}, \quad x_{1}^{*}, \ldots, x_{n}^{*} \in \mathcal{X}^{*} .
$$

(ii): Let $\wedge^{* 0} \mathcal{X} \doteq \mathbb{C}$, while, for all $n \in \mathbb{N}$, we use the definition

$$
\wedge^{* n} \mathcal{X} \doteq \operatorname{lin}\left\{x_{1}^{*} \wedge \cdots \wedge x_{n}^{*}: x_{1}^{*}, \ldots, x_{n}^{*} \in \mathcal{X}^{*}\right\} .
$$

We then define the vector space

$$
\wedge^{*} \mathcal{X} \doteq \bigoplus_{n=0}^{\infty} \wedge^{* n} \mathcal{X}
$$

Recall that the infinite direct sum of a family $\left\{\mathcal{X}_{n}\right\}_{n \in \mathbb{N}}$ of vector spaces, like in the above definition, is the subspace of the product space $\prod_{n=0}^{\infty} \mathcal{X}_{n}$, the elements of which are sequences that eventually vanish. (iii): For $n, m \in \mathbb{N}_{0}, \xi \in \wedge^{* n} \mathcal{X}$ and $\zeta \in \wedge^{* m} \mathcal{X}$, their exterior product $\xi \wedge \zeta \in \wedge^{* n+m} \mathcal{X}$ is defined by

$$
\xi \wedge \zeta\left(x_{1}, \ldots, x_{n+m}\right) \doteq \frac{1}{n ! m !} \sum_{\pi \in \mathcal{S}_{n+m}}(-1)^{\pi} \xi\left(x_{\pi(1)}, \ldots, x_{\pi(n)}\right) \zeta\left(x_{\pi(n+1)}, \ldots, x_{\pi(n+m)}\right)
$$

where $\mathcal{S}_{N}$ is the set of all permutations of $N \in \mathbb{N}$ elements. This prescription uniquely defines an associative product on $\wedge^{*} \mathcal{X}$, which is consistent with the definition of the $n$-linear form (33):

$$
x_{1}^{*} \wedge\left(x_{2}^{*} \wedge \cdots \wedge x_{n}^{*}\right)=x_{1}^{*} \wedge \cdots \wedge x_{n}^{*}, \quad n \in \mathbb{N}, x_{1}^{*}, \ldots, x_{n}^{*} \in \mathcal{X}^{*} .
$$

Compare (37) with (33), using the Leibniz formula to compute the determinant. 


\section{Definition 3.1 (Grassmann algebra)}

The Grassmann algebra on a topological vector space $\mathcal{X}$ is the (associative and distributive) algebra $\left(\wedge^{*} \mathcal{X},+, \wedge\right)$.

\section{Notation 3.2}

When there is no risk of ambiguity, we use $x_{1}^{*} \wedge \cdots \wedge x_{n}^{*} \equiv x_{1}^{*} \cdots x_{n}^{*}$ to denote exterior products.

For $n \in \mathbb{N}_{0}, \wedge^{* n} \mathcal{X}$ is precisely the subspace of elements of degree $n$ of the graded algebra $\wedge^{*} \mathcal{X}$. The unit of the Grassmann algebra $\wedge^{*} \mathcal{X}$ is denoted by

$$
1 \doteq 1 \in \wedge^{* 0} \mathcal{X} \subset \wedge^{*} \mathcal{X}
$$

and $[\xi]_{0}$ stands for the zero-degree component of any element $\xi$ of $\wedge^{*} \mathcal{X}$.

The exponential function in Grassmann algebra is important in the sequel. It is defined, as is usual, by

$$
\exp (\xi) \doteq \mathbf{1}+\sum_{k=1}^{\infty} \frac{\xi^{k}}{k !}, \quad \xi \in \wedge^{*} \mathcal{X} .
$$

This function is well-defined because, by definition of $\wedge^{*} \mathcal{X}, \xi \in \wedge^{*} \mathcal{X}$ is contained in some finitedimensional subalgebra of $\wedge^{*} \mathcal{X}$. Indeed, by finite dimensionality, for any norm on the space $\wedge^{*} \mathcal{X}$ and any $\xi \in \wedge^{*} \mathcal{X}$, there is a constant $D_{\xi}<\infty$ such that

$$
\left\|\xi^{k}\right\|_{\wedge^{* \mathcal{X}}} \leq\left(D_{\xi}\right)^{k}, \quad k \in \mathbb{N} .
$$

Hence, the series defining $\exp (\xi)$ is absolutely convergent in the normed space $\wedge^{*} \mathcal{X}$.

In the sequel, we use $\mathcal{X}=\mathcal{H}$. In this case, the linear spaces $\operatorname{sCAR}(\mathcal{H}, \mathfrak{A})$ and $\wedge^{*} \mathcal{H}$ are isomorphic to each other because they have exactly the same dimension:

$$
\operatorname{dim}(\operatorname{sCAR}(\mathcal{H}, \mathfrak{A}))=2^{\operatorname{dim} \mathcal{H}}=\operatorname{dim}\left(\wedge^{*} \mathcal{H}\right)<\infty .
$$

However, $\left(\wedge^{*} \mathcal{H},+, \wedge\right)$ is not isomorphic to a self-dual CAR algebra over $\mathcal{H}$, because of the CAR (11). We thus define below a new product and an involution to make $\wedge^{*} \mathcal{H}$ a self-dual CAR algebra $\operatorname{sCAR}(\mathcal{H}, \mathfrak{A})$. This explicit construction uses Berezin derivatives and integrals, which are meanwhile pivotal to obtain the Brydges-Kennedy tree expansion of the Gärtner-Ellis generating functions.

\subsection{Berezin Integral}

As a preliminary step, we introduce the notion of Berezin derivatives. For any $\varphi \in \mathcal{H}$, it is the linear operator $\delta / \delta \varphi$ acting on the Grassmann algebra $\wedge^{*} \mathcal{H}$ that is uniquely defined by the conditions

$$
\frac{\delta}{\delta \varphi} \tilde{\varphi}=\langle\varphi, \tilde{\varphi}\rangle_{\mathcal{H}} \mathbf{1} \quad \text { and } \quad \frac{\delta}{\delta \varphi} \xi_{1} \xi_{2}=\left(\frac{\delta}{\delta \varphi} \xi_{1}\right) \wedge \xi_{2}+(-1)^{n} \xi_{1} \wedge\left(\frac{\delta}{\delta \varphi} \xi_{2}\right),
$$

for any $\tilde{\varphi} \in \mathcal{H}$ and element $\xi_{1} \in \wedge^{* n} \mathcal{H}$ of degree $n \in \mathbb{N}$, and all $\xi_{2} \in \wedge^{*} \mathcal{H}$. In particular, the map $\varphi \mapsto \delta / \delta \varphi$ from $\mathcal{H}$ to $\mathcal{B}\left(\wedge^{*} \mathcal{H}\right)$ is antilinear and satisfies

$$
\frac{\delta}{\delta \varphi_{1}} \frac{\delta}{\delta \varphi_{2}}=-\frac{\delta}{\delta \varphi_{2}} \frac{\delta}{\delta \varphi_{1}}, \quad \varphi_{1}, \varphi_{2} \in \mathcal{H}
$$

Note that $\delta / \delta \varphi$ is an annihilation operator on $\wedge^{*} \mathcal{H}$, viewed as the fermionic Fock space $\mathcal{F}_{\mathcal{H}^{*}}$. See Section 6.4. Viewed this way, $\delta / \delta \varphi \doteq(\mathfrak{A} \varphi)\lrcorner$ for any $\varphi \in \mathcal{H}$. 
Recall that, for each $k \in \mathbb{N}_{0}, \mathcal{X}^{(k)}$ denotes a copy of some vector space $\mathcal{X}$ and the corresponding copy of $\xi \in \mathcal{X}$ is written as $\xi^{(k)}$, see Notation 1.3 (ii). For any $K \subset\{0, \ldots, N\}$ with $N \in \mathbb{N}_{0}$, we identify $\wedge^{*}\left(\oplus_{k \in K} \mathcal{H}^{(k)}\right)$ with the Grassmann subalgebra of $\wedge^{*}\left(\oplus_{k=0}^{N} \mathcal{H}^{(k)}\right)$ generated by the union

$$
\bigcup_{k \in K}\left\{\varphi^{(k)}: \varphi \in \mathcal{H}\right\}
$$

We meanwhile identify $\wedge^{*} \mathcal{H}^{(0)}$ with the Grassmann algebra $\wedge^{*} \mathcal{H}$, i.e.,

$$
\wedge^{*} \mathcal{H}^{(0)} \equiv \wedge^{*} \mathcal{H}
$$

We are now in a position to define the so-called Berezin integral [B66, Section I.3]:

\section{Definition 3.3 (Berezin integral)}

Fix $N \in \mathbb{N}_{0}$ and a basis projection $P$ (Definition 2.4) with $\left\{\psi_{i}\right\}_{i \in J}$ being any orthonormal basis of its range $\mathfrak{h}_{P}$. For all $k \in\{0, \ldots, N\}$, we define the linear map

$$
\int_{P} \mathrm{~d}\left(\mathcal{H}^{(k)}\right): \wedge^{*}\left(\oplus_{q=0}^{N} \mathcal{H}^{(q)}\right) \rightarrow \wedge^{*}\left(\oplus_{q \in\{0, \ldots, N\} \backslash\{k\}} \mathcal{H}^{(q)}\right)
$$

by

$$
\int_{P} \mathrm{~d}\left(\mathcal{H}^{(k)}\right) \doteq \prod_{i \in J}\left(\frac{\delta}{\delta \psi_{i}^{(k)}} \frac{\delta}{\delta\left(\left(\mathfrak{A} \psi_{i}\right)^{(k)}\right)}\right)
$$

For $N=0$, the Berezin integral defines a linear form from $\wedge^{*} \mathcal{H}^{(0)} \equiv \wedge^{*} \mathcal{H}$ to $\mathbb{C}_{\mathbf{1}} \equiv \mathbb{C}$. Recall that usual integrals can be seen as linear forms on an algebra of continuous functions. This partially justifies the use of the term "integral" for the map $\int_{P} \mathrm{~d}\left(\mathcal{H}^{(k)}\right)$.

The product defining the Berezin integral, with index set $J$, does not depend on the order of its terms, because of (42). For any fixed basis projection $P$, the Berezin integral also does not depend on the particular choice of the orthonormal basis $\left\{\psi_{i}\right\}_{i \in J}$ of $\mathfrak{h}_{P}$ : Considering the case $N=0$ without loss of generality, for any unitary transformation $U$ on $\mathfrak{h}_{P}$, observe that, for all $i \in J$,

$$
\frac{\delta}{\delta\left(U \psi_{i}\right)}=\sum_{j \in J}\left\langle U \psi_{i}, \psi_{j}\right\rangle_{\mathcal{H}} \frac{\delta}{\delta \psi_{j}} \quad \text { and } \quad \frac{\delta}{\delta\left(\mathfrak{A} U \psi_{i}\right)}=\sum_{j \in J}\left\langle\psi_{j}, U \psi_{i}\right\rangle_{\mathcal{H}} \frac{\delta}{\delta\left(\mathfrak{A} \psi_{j}\right)}
$$

Therefore, by using the fact that Berezin derivatives anticommute with each other, one directly computes that

$$
\prod_{i \in J}\left(\frac{\delta}{\delta\left(U \psi_{i}\right)} \frac{\delta}{\delta\left(\mathfrak{A} U \psi_{i}\right)}\right)=\prod_{i \in J}\left(\frac{\delta}{\delta\left(\psi_{i}\right)} \frac{\delta}{\delta\left(\mathfrak{A} \psi_{i}\right)}\right)
$$

For two different basis projections, the corresponding Berezin integrals can, at most, differ by a sign:

\section{Lemma 3.4 (Orientation of Berezin integrals)}

For any basis projections $P_{1}, P_{2} \in \mathcal{B}(\mathcal{H})$, there exists $\varepsilon \equiv \varepsilon\left(P_{1}, P_{2}\right) \in\{-1,1\}$ such that, for all $N \in \mathbb{N}_{0}$ and $k \in\{0, \ldots, N\}$,

$$
\int_{P_{1}} \mathrm{~d}\left(\mathcal{H}^{(k)}\right)=\varepsilon \int_{P_{2}} \mathrm{~d}\left(\mathcal{H}^{(k)}\right) .
$$

Proof. By [A68, Lemma 3.6], for any pair of basis projections $P_{1}, P_{2} \in \mathcal{B}(\mathcal{H})$ there is a (generally not unique) Bogoliubov transformation $U$ such that $P_{2}=U P_{1} U^{*}$. In particular, by using orthonormal bases $\left\{\psi_{i}^{(n)}\right\}_{i \in J}$ of $\mathfrak{h}_{P_{n}}, n=1,2$, and identities similar to (44) one gets

$$
\int_{P_{1}} \mathrm{~d}\left(\mathcal{H}^{(k)}\right)=\operatorname{det}(U) \int_{P_{2}} \mathrm{~d}\left(\mathcal{H}^{(k)}\right)
$$


for some Bogoliubov transformation $U$. Therefore, by (16), the assertion follows.

We therefore say that the corresponding Berezin integrals, and hence the associated Basis projections, have the same orientation if $\varepsilon=1$. Note that this statement shows that the orientation of any Bogoliubov transformation $U$ such that $P_{2}=U P_{1} U^{*}$ only depends on the basis projections $P_{1}, P_{2}$.

\subsection{Bilinear Elements of Grassmann Algebra}

By using Notation 1.3 (ii), we define bilinear elements of Grassmann algebra in a similar way to bilinear elements of self-dual CAR algebra (Definition 2.5):

\section{Definition 3.5 (Bilinear elements of Grassmann algebras)}

Let $\mathcal{H}$ be any finite-dimensional self-dual Hilbert space with antiunitary involution $\mathfrak{A}$.

(i) Fix an orthonormal basis $\left\{\psi_{i}\right\}_{i \in I}$ of $\mathcal{H}$ and define, for all $H \in \mathcal{B}(\mathcal{H})$, a bilinear element of the Grassmann algebra $\wedge^{*} \mathcal{H}$ by

$$
\langle\mathcal{H}, H \mathcal{H}\rangle \doteq \sum_{i, j \in I}\left\langle\psi_{i}, H \psi_{j}\right\rangle_{\mathcal{H}}\left(\mathfrak{A} \psi_{j}\right) \wedge \psi_{i}
$$

(ii) Fix a basis projection $P$ associated with $(\mathcal{H}, \mathfrak{A})$ and an orthonormal basis $\left\{\psi_{j}\right\}_{j \in J}$ of its range $\mathfrak{h}_{P}$. Given $k, l \in \mathbb{N}_{0}$,

$$
\left\langle\mathfrak{h}_{P}^{(k)}, \mathfrak{h}_{P}^{(l)}\right\rangle \doteq \sum_{j \in J}\left(\mathfrak{A} \psi_{j}\right)^{(k)} \wedge \psi_{j}^{(l)}
$$

This definition does not depend on the particular choice of the orthonormal basis. Compare Definition 3.5 with Definition 2.5. Additionally, note that

$$
\langle\mathcal{H}, H \mathcal{H}\rangle=\sum_{i, j \in I}\left\langle\psi_{i}, H \mathfrak{A} \psi_{j}\right\rangle_{\mathcal{H}} \psi_{j} \wedge \psi_{i}=\sum_{i, j \in I}\left\langle\mathfrak{A} \psi_{i}, H \psi_{j}\right\rangle_{\mathcal{H}}\left(\mathfrak{A} \psi_{j}\right) \wedge \mathfrak{A} \psi_{i}
$$

for all $H \in \mathcal{B}(\mathcal{H})$.

For any basis projection $P$ diagonalizing a self-dual operator $H$ on $(\mathcal{H}, \mathfrak{A})$ (see Definition 2.7 and (23)) and any orthonormal basis $\left\{\psi_{j}\right\}_{j \in J}$ of $\mathfrak{h}_{P}$,

$$
\langle\mathcal{H}, H \mathcal{H}\rangle=2 \sum_{i, j \in J}\left\langle\psi_{i}, H \psi_{j}\right\rangle_{\mathcal{H}}\left(\mathfrak{A} \psi_{j}\right) \wedge \psi_{i}
$$

Compare this assertion with the first term of the right-hand side of Equation (24). Notice also that

$$
\langle\mathcal{H}, \mathbf{K}(P) \mathcal{H}\rangle=\frac{1}{2}\left\langle\mathcal{H},\left(P-P^{\perp}\right) \mathcal{H}\right\rangle=\left\langle\mathfrak{h}_{P}, \mathfrak{h}_{P}\right\rangle .
$$

See Equation (21).

Bilinear Hamiltonians of self-dual CAR algebra are used to fix a quasi-free state, as explained in Lemma 2.10. Similarly, we employ the exponential function (38) and bilinear elements of Grassmann algebra to define Gaussian Berezin integrals:

For any basis projection $P$ diagonalizing a self-dual operator $H$ on $(\mathcal{H}, \mathfrak{A})$, elementary computations yield

$$
\int_{P} \mathrm{~d}(\mathcal{H}) \mathrm{e}^{\langle\mathcal{H}, H \mathcal{H}\rangle}=\operatorname{det}\left(H_{P}\right)
$$

In particular, $\operatorname{det}\left(H_{P}\right)$ only depends on $H$ and the orientation of $P$, by Lemma 3.4. Gaussian Berezin integrals are then defined as follows: 


\section{Definition 3.6 (Gaussian Berezin integrals)}

Let $C \in \mathcal{B}(\mathcal{H})$ be any invertible self-dual operator. The Gaussian Berezin integral with covariance $C \in \mathcal{B}(\mathcal{H})$ is the linear map $\int \mathrm{d} \mu_{C}(\mathcal{H})$ from $\wedge^{*} \mathcal{H}$ to $\mathbb{C} 1$ defined by

$$
\int \mathrm{d} \mu_{C}(\mathcal{H}) \xi \doteq \operatorname{det}\left(\frac{C_{P}}{2}\right) \int_{P} \mathrm{~d}(\mathcal{H}) \mathrm{e}^{\frac{1}{2}\left\langle\mathcal{H}, C^{-1} \mathcal{H}\right\rangle} \wedge \xi, \quad \xi \in \wedge^{*} \mathcal{H}
$$

where $P$ is any basis projection diagonalizing $C$.

Note that $C^{-1}$ is a self-dual operator whenever $C$ is invertible and self-dual. In this case, if $P$ diagonalizes $C$ then it also diagonalizes $C^{-1}$. Observe in the above definition that $\left(C_{P} / 2\right)^{-1}=$ $\left(C^{-1}\right)_{P} / 2$.

Remark further that, by Lemma 3.4 and Equation (47), Gaussian Berezin integrals do not depend on the particular choice of the basis projection $P$ in Definition 3.6. They have properties that are reminiscent of those of quasi-free states (see, e.g., (26)-(27)):

\section{Proposition 3.7 (Gaussian Berezin integrals as Pfaffians)}

Let $C \in \mathcal{B}(\mathcal{H})$ be any invertible self-dual operator. Then, $\int \mathrm{d} \mu_{C}(\mathcal{H}) 1=1$ while, for all $N \in \mathbb{N}_{0}$ and $\varphi_{0}, \ldots, \varphi_{2 N} \in \mathcal{H}$,

$$
\int \mathrm{d} \mu_{C}(\mathcal{H}) \varphi_{0} \cdots \varphi_{2 N}=0 \text { and } \int \mathrm{d} \mu_{C}(\mathcal{H}) \varphi_{1} \cdots \varphi_{2 N}=\operatorname{Pf}\left[\left\langle\mathfrak{A} \varphi_{k}, C \varphi_{l}\right\rangle_{\mathcal{H}}\right]_{k, l=1}^{2 N} \mathbf{l}
$$

where Pf is the Pfaffian defined, for any skew-symmetric matrix, by (28).

Proof. This kind of identity is well-known, see, e.g., [FKT02, Proposition 1.19]. We give its proof for completeness. Observe that $\left[\left\langle\mathfrak{A} \varphi_{k}, C \varphi_{l}\right\rangle_{\mathcal{H}}\right]_{k, l=1}^{2 N}$ is a skew-symmetric matrix because $C \in \mathcal{B}(\mathcal{H})$ is a self-dual operator. $\int \mathrm{d} \mu_{C}(\mathcal{H}) \perp=1$ is an obvious consequence of (47), while the first identity of (48) directly follows from the definition of Berezin integrals. The proof of the second identity of (48) is less direct. It is performed in three steps for any invertible self-dual operator $C \in \mathcal{B}(\mathcal{H})$ and basis projection $P$ diagonalizing $C$ :

Step 1: For any orthonormal basis $\left\{\psi_{i}\right\}_{i \in I}$ of $\mathcal{H}$, let

$$
\left\langle\mathcal{H}^{(0)}, \mathcal{H}^{(1)}\right\rangle \doteq \sum_{i \in I}\left(\mathfrak{A} \psi_{i}^{(0)}\right) \wedge \psi_{i}^{(1)}=-\sum_{i \in I}\left(\mathfrak{A} \psi_{i}^{(1)}\right) \wedge \psi_{i}^{(0)}
$$

Observe that this Grassmann algebra element does not depend on the special choice of the orthonormal basis. Moreover,

$$
\varphi^{(1)}=\frac{\delta}{\delta\left(\mathfrak{A} \varphi^{(0)}\right)}\left\langle\mathcal{H}^{(0)}, \mathcal{H}^{(1)}\right\rangle, \quad \varphi \in \mathcal{H}
$$

It follows that, for all $N \in \mathbb{N}$ and $\varphi_{1}, \ldots, \varphi_{2 N} \in \mathcal{H}$,

$$
\int \mathrm{d} \mu_{C}(\mathcal{H}) \varphi_{1} \cdots \varphi_{2 N}=\left[\left(\prod_{k=1}^{2 N} \frac{\delta}{\delta\left(\mathfrak{A} \varphi_{k}^{(0)}\right)}\right) \int \mathrm{d} \mu_{C}\left(\mathcal{H}^{(1)}\right) \mathrm{e}^{\left\langle\mathcal{H}^{(0)}, \mathcal{H}^{(1)}\right\rangle}\right]_{0},
$$

recalling that $[\xi]_{0}$ denotes the zero-degree component of any element $\xi$ of the Grassmann (graded) algebra $\wedge^{*} \mathcal{H} \equiv \wedge^{*} \mathcal{H}^{(0)}$.

Step 2: For $s \in\{0,1\}$, define the Grassmann algebra element

$$
\mathbf{A}_{s} \doteq \frac{1}{2} \sum_{i, j \in I}\left\langle\psi_{i}, C^{-1} \psi_{j}\right\rangle_{\mathcal{H}} \Phi_{s, j}^{(1,0)} \wedge \Psi_{s, i}^{(1,0)}+\sum_{i \in I}\left(\mathfrak{A} \psi_{i}^{(0)}\right) \wedge \Psi_{s, i}^{(1,0)}
$$


where, for any $i \in I$,

$$
\Psi_{s, i}^{(1,0)} \doteq \psi_{i}^{(1)}+s \sum_{j \in I}\left\langle\psi_{j}, C \psi_{i}\right\rangle_{\mathcal{H}} \psi_{j}^{(0)}, \quad \Phi_{s, i}^{(1,0)} \doteq \mathfrak{A} \psi_{i}^{(1)}-s \sum_{j \in I}\left\langle\psi_{i}, C \psi_{j}\right\rangle_{\mathcal{H}} \mathfrak{A} \psi_{j}^{(0)} .
$$

Using Definition 3.5 (i) and (49), for $s \in\{0,1\}$,

$$
\mathbf{A}_{s}=\frac{1}{2}\left\langle\mathcal{H}^{(1)}, C^{-1} \mathcal{H}^{(1)}\right\rangle+(1-s)\left\langle\mathcal{H}^{(0)}, \mathcal{H}^{(1)}\right\rangle+\frac{s}{2}\left\langle\mathcal{H}^{(0)}, C \mathcal{H}^{(0)}\right\rangle .
$$

Direct computations then show that, for any basis projection $P$,

$$
\int_{P} \mathrm{~d}\left(\mathcal{H}^{(1)}\right) \mathrm{e}^{\mathbf{A}_{0}}=\int_{P} \mathrm{~d}\left(\mathcal{H}^{(1)}\right) \mathrm{e}^{\mathbf{A}_{1}} .
$$

Combined with (47) and (50), these last two equations yield

$$
\int \mathrm{d} \mu_{C}(\mathcal{H}) \varphi_{1} \cdots \varphi_{2 N}=\frac{1}{2^{N} N !}\left(\prod_{k=1}^{2 N} \frac{\delta}{\delta\left(\mathfrak{A} \varphi_{k}\right)}\right)\langle\mathcal{H}, C \mathcal{H}\rangle^{N}
$$

for any $N \in \mathbb{N}$ and $\varphi_{1}, \ldots, \varphi_{2 N} \in \mathcal{H}$.

Step 3: To compute the right-hand side of (51), it is convenient to take $\varphi_{1}, \ldots, \varphi_{2 N}$ from an orthonor-

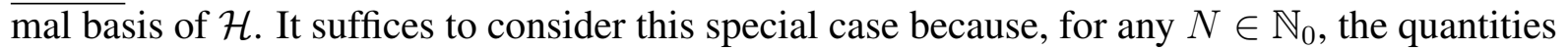

$$
\int \mathrm{d} \mu_{C}(\mathcal{H}) \varphi_{1} \cdots \varphi_{2 N} \quad \text { and } \operatorname{Pf}\left[\left\langle\mathfrak{A} \varphi_{k}, C \varphi_{l}\right\rangle_{\mathcal{H}}\right]_{k, l=1}^{2 N} \mathbf{l}
$$

are linear with respect to $\varphi_{1}, \ldots, \varphi_{2 N} \in \mathcal{H}$. So, fix an orthonormal basis $\left\{\psi_{i}\right\}_{i \in I}$ of $\mathcal{H}$ and any index set $\left\{i_{1}, \ldots, i_{2 N}\right\} \subset I$ for $N \in \mathbb{N}$. Then, we infer from (49) and (51) that

$$
\begin{aligned}
& \int \mathrm{d} \mu_{C}(\mathcal{H}) \psi_{i_{1}} \cdots \psi_{i_{2 N}} \\
= & \frac{1}{2^{N} N !}\left(\prod_{k=1}^{2 N} \frac{\delta}{\delta\left(\mathfrak{A} \psi_{i_{k}}\right)}\right) \sum_{\pi \in \mathcal{S}_{2 N}} \prod_{k=1}^{N}\left\langle\mathfrak{A} \psi_{i_{\pi(2 k-1)}}, C \psi_{i_{\pi(2 k)}}\right\rangle_{\mathcal{H}}\left(\mathfrak{A} \psi_{i_{\pi(2 k)}}\right) \wedge\left(\mathfrak{A} \psi_{i_{\pi(2 k-1)}}\right) \\
= & \left(\prod_{k=1}^{2 N} \frac{\delta}{\delta\left(\mathfrak{A} \psi_{i_{k}}\right)}\right) \sum_{\pi \in \mathcal{S}_{2 N}} \frac{(-1)^{\pi}}{2^{N} N !} \prod_{k=1}^{N}\left\langle\mathfrak{A} \psi_{i_{\pi(2 k-1)}}, C \psi_{i_{\pi(2 k)}}\right\rangle_{\mathcal{H}}\left(\mathfrak{A} \psi_{i_{2(N-k+1)}}\right) \wedge\left(\mathfrak{A} \psi_{i_{2(N-k+1)-1}}\right),
\end{aligned}
$$

which yields the Pfaffian we are looking for.

\section{Corollary 3.8 (Gaussian Berezin integrals as determinants)}

Let $C \in \mathcal{B}(\mathcal{H})$ be any invertible self-dual operator and fix any basis projection $P$ diagonalizing $C$. Then, for all $N \in \mathbb{N}$ and $\varphi_{1}, \ldots, \varphi_{2 N} \in \mathfrak{h}_{P}$,

$$
\int \mathrm{d} \mu_{C}(\mathcal{H})\left(\mathfrak{A} \varphi_{1}\right) \cdots\left(\mathfrak{A} \varphi_{N}\right) \varphi_{2 N} \cdots \varphi_{N+1}=\operatorname{det}\left[\left\langle\varphi_{k}, C \varphi_{N+l}\right\rangle_{\mathcal{H}}\right]_{k, l=1}^{N} \mathbf{1}
$$

Proof. As, by assumption, $P$ is a basis projection diagonalizing the self-dual operator $C$, this follows from Proposition 3.7 together with the following identity for the Pfaffian:

$$
\operatorname{Pf}\left(\begin{array}{cc}
0 & M \\
-M^{\mathrm{t}} & 0
\end{array}\right)=(-1)^{N(N-1) / 2} \operatorname{det}(M),
$$

for any matrix $M \in \operatorname{Mat}(N, \mathbb{C}), N \in \mathbb{N}$, where $M^{\mathrm{t}}$ denotes its transpose.

Compare Proposition 3.7 with Equations (26)-(27) and Lemma 2.10. In fact, the strong analogy between bilinear elements of self-dual CAR algebra together with their associated quasi-free states and bilinear elements of Grassmann algebra together with their Gaussian Berezin integrals is essential to derive Pfaffian bounds in Section 5. 


\subsection{From Grassmann Algebra to Self-Dual CAR Algebra}

We can now define a "circle" product $\circ$ and an involution * on $\wedge^{*} \mathcal{H}$ to make a self-dual CAR algebra (Definition 2.1) out of this space. To this end, we take any basis projection $P$ with range $\mathfrak{h}_{P}$. See Definition 2.4 and Equation (13). Then, for all $i, j, k, l \in \mathbb{N}_{0}$, we define by

$$
\varkappa_{(i, j)}^{(k, l)}: \wedge^{*}\left(\mathfrak{h}_{P}^{(i)} \oplus \mathfrak{h}_{P}^{*(j)}\right) \rightarrow \wedge^{*}\left(\mathfrak{h}_{P}^{(k)} \oplus \mathfrak{h}_{P}^{*(l)}\right)
$$

the unique isomorphism of linear spaces such that $\varkappa_{(i, j)}^{(k, l)}(z \mathbf{1})=z \mathbf{1}$ for $z \in \mathbb{C}$ and, for any $m, n \in \mathbb{N}_{0}$ so that $m+n \geq 1$, and all $\varphi_{1}, \ldots, \varphi_{m+n} \in \mathfrak{h}_{P}$,

$$
\varkappa_{(i, j)}^{(k, l)}\left(\left(\mathfrak{A} \varphi_{1}\right)^{(i)} \cdots\left(\mathfrak{A} \varphi_{m}\right)^{(i)} \varphi_{m+1}^{(j)} \cdots \varphi_{m+n}^{(j)}\right)=\left(\mathfrak{A} \varphi_{1}\right)^{(k)} \cdots\left(\mathfrak{A} \varphi_{m}\right)^{(k)} \varphi_{m+1}^{(l)} \cdots \varphi_{m+n}^{(l)}
$$

with $\varphi_{1} \wedge \varphi_{2} \equiv \varphi_{1} \varphi_{2}$ (see Notation 1.3 (ii) and 3.2). Here, if $m=0$, then there is no $\mathfrak{A} \varphi$ in the above equation. Mutatis mutandis for $n=0$. Note that $\varkappa_{(i, j)}^{(k, l)}$ strongly depends on the choice of the basis projection $P$ even if we overlook the symbol $P$ in the notation of this isomorphism.

By using the objects defined by (38), Definition 3.5 and (53)-(54), as well as Berezin integrals (Definition 3.3), we introduce a new product on $\wedge^{*} \mathcal{H}$ depending on a basis projection:

\section{Definition 3.9 (Circle products with respect to basis projections)}

Fix a basis projection $P$ with range $\mathfrak{h}_{P}$ and recall (13), that is, $\mathcal{H} \equiv \mathfrak{h}_{P} \oplus \mathfrak{h}_{P}^{*}$. For any $\xi_{0}, \xi_{1} \in \wedge^{*} \mathcal{H}$, we define their circle product by

$$
\xi_{0} \circ_{P} \xi_{1} \doteq(-1)^{\frac{\operatorname{dim} \mathcal{H}}{2}} \int_{P} \mathrm{~d}\left(\mathcal{H}^{(1)}\right) \varkappa_{(0,0)}^{(0,1)}\left(\xi_{0}\right) \varkappa_{(0,0)}^{(1,0)}\left(\xi_{1}\right) \mathrm{e}^{-\left\langle\mathfrak{h}_{P}^{(0)}, \mathfrak{h}_{P}^{(0)}\right\rangle} \mathrm{e}^{\left\langle\mathfrak{h}_{P}^{(0)}, \mathfrak{h}_{P}^{(1)}\right\rangle} \mathrm{e}^{-\left\langle\mathfrak{h}_{P}^{(1)}, \mathfrak{h}_{P}^{(1)}\right\rangle} \mathrm{e}^{\left\langle\mathfrak{h}_{P}^{(1)}, \mathfrak{h}_{P}^{(0)}\right\rangle} .
$$

It directly follows from this definition that the circle product is associative and distributive on the space $\wedge^{*} \mathcal{H}$ with the same unit $\perp$ as with the exterior product $\wedge$ :

\section{Lemma 3.10 (Elementary properties of the circle product)}

Fix a basis projection $P$ with range $\mathfrak{h}_{P}$. Then, the circle product $\circ_{P}$ has the following properties:

(i) Associativity: For all $N \in \mathbb{N}, N \geq 2$, and $\xi_{0}, \ldots, \xi_{N-1} \in \wedge^{*} \mathcal{H}$,

$$
\begin{aligned}
\xi_{0} \circ_{P} \cdots \circ_{P} \xi_{N-1}=(-1)^{(N-1) \frac{\operatorname{dim} \mathcal{H}}{2}}\left(\prod_{k=1}^{N-1} \int_{P} \mathrm{~d}\left(\mathcal{H}^{(k)}\right)\right)\left(\prod_{k=0}^{N-1} \varkappa_{(0,0)}^{(k, k+1 \bmod N)}\left(\xi_{k}\right)\right) \\
\prod_{k=0}^{N-1} \mathrm{e}^{-\left\langle\mathfrak{h}_{P}^{(k)}, \mathfrak{h}_{P}^{(k)}\right\rangle} \mathrm{e}^{\left\langle\mathfrak{h}_{P}^{(k)}, \mathfrak{h}_{P}^{(k+1 \bmod N)}\right\rangle} .
\end{aligned}
$$

(ii) Distributivity over the addition: For $n_{1}, n_{2} \in \mathbb{N}, z_{1}, \ldots, z_{n_{1}+n_{2}} \in \mathbb{C}$, and $\xi_{1}, \ldots, \xi_{n_{1}+n_{2}} \in \wedge^{*} \mathcal{H}$,

$$
\left(\sum_{j=1}^{n_{1}} z_{j} \xi_{j}\right) \circ_{P}\left(\sum_{j=1}^{n_{2}} z_{j+n_{1}} \xi_{j+n_{1}}\right)=\sum_{j_{1}=1}^{n_{1}} \sum_{j_{2}=1}^{n_{2}}\left(z_{j_{1}} z_{j_{2}+n_{1}}\right) \xi_{j_{1}} \circ_{P} \xi_{j_{2}+n_{1}} .
$$

(iii) The unit of the circle product coincides with the unit 1 of the exterior product $\wedge$ :

$$
z \mathbf{1} \circ_{P} \xi=\xi \circ_{P} z \mathbf{1}=z \xi, \quad z \in \mathbb{C}, \xi \in \wedge^{*} \mathcal{H} .
$$

(iv) Clustering property: For any subspace $\mathcal{Y} \subset \mathcal{H}$ with orthogonal complement $\mathcal{Y}^{\perp}$,

$$
\xi_{0} \circ_{P} \xi_{1}=\xi_{0} \wedge \xi_{1}, \quad \xi_{0} \in \wedge^{*} \mathfrak{A}(\mathcal{Y}), \xi_{1} \in \wedge^{*} \mathcal{Y}^{\perp}
$$


(v) Canonical Anti-commutation Relations (CAR): For any $N \in \mathbb{N}$ and $\varphi_{1}, \ldots, \varphi_{N+1} \in \mathcal{H}$, one has ${ }^{13}$

$$
\left(\varphi_{1} \wedge \cdots \wedge \varphi_{N}\right) \circ_{P} \varphi_{N+1}=\varphi_{1} \wedge \cdots \wedge \varphi_{N+1}+\sum_{q=1}^{N}(-1)^{N-q}\left\langle\mathfrak{A} \varphi_{q}, P^{\perp} \varphi_{N+1}\right\rangle_{\mathcal{H}} \prod_{m \in\{1, \ldots, N\} \backslash\{q\}} \varphi_{m} .
$$

(vi) Wedge expansion of the antisymmetrized circle product: For $N \in \mathbb{N} \backslash\{1\}$ and $\varphi_{1}, \ldots, \varphi_{N} \in \mathcal{H}$,

$$
\begin{array}{r}
\frac{1}{N !} \sum_{\pi \in \mathcal{S}_{N}}(-1)^{\pi} \varphi_{\pi(1)} \circ_{P} \cdots \circ_{P} \varphi_{\pi(N)}=\sum_{\mathcal{N} \subseteq\{1, \ldots, N\},|\mathcal{N}| \in 2 \mathbb{N}_{0}} 2^{-\frac{|\mathcal{N}|}{2}} \operatorname{Pf}\left[\left\langle\mathfrak{A} \varphi_{k}, \frac{P^{\perp}-P}{2} \varphi_{l}\right\rangle_{\mathcal{H}}\right]_{k, l \in \mathcal{N}} \\
\operatorname{sign}(\mathcal{N},\{1, \ldots, N\}) \prod_{m \in\{1, \ldots, N\} \backslash \mathcal{N}} \varphi_{m},
\end{array}
$$

where the product on the right-hand side is $\wedge$ (see Notation 3.2),

$$
\operatorname{Pf}\left[\left\langle\mathfrak{A} \varphi_{k}, \frac{P^{\perp}-P}{2} \varphi_{l}\right\rangle_{\mathcal{H}}\right]_{k, l \in \emptyset} \doteq 1
$$

and, for any finite subsets $\mathcal{M} \subseteq \mathbb{N}$ and $\mathcal{N} \subseteq \mathcal{M}$, $\operatorname{sign}(\mathcal{N}, \mathcal{M})$ is the signature of the unique permutation of $\mathcal{M}$ which takes all elements of $\mathcal{N}$ to the left in such a way that they are ordered.

Proof. Assertions (i)-(iv) are deduced from straightforward, albeit sometimes cumbersome, computations using Definitions 2.4, 3.3, 3.5 and 3.9, Equations (53)-(54), together with the properties given by (34) and (41)-(42). We now prove Assertion (v), starting with elementary observations: By using (34), observe from (38) and Definition 3.5 that, for any $k, l \in \mathbb{N}_{0}$ and basis projection $P$,

$$
\mathrm{e}^{ \pm\left\langle\mathfrak{h}_{P}^{(k)}, \mathfrak{h}_{P}^{(l)}\right\rangle}=\prod_{j \in J} \mathrm{e}^{ \pm\left(\mathfrak{A} \psi_{j}\right)^{(k)} \wedge \psi_{j}^{(l)}}=\prod_{j \in J}\left(1 \pm\left(\mathfrak{A} \psi_{j}\right)^{(k)} \wedge \psi_{j}^{(l)}\right)
$$

Here, $\left\{\psi_{j}\right\}_{j \in J}$ is any orthonormal basis of the range $\mathfrak{h}_{P}$ of $P$. In particular,

$$
\begin{aligned}
& \mathrm{e}^{-\left\langle\mathfrak{h}_{P}^{(0)}, \mathfrak{h}_{P}^{(0)}\right\rangle} \mathrm{e}^{\left\langle\mathfrak{h}_{P}^{(0)}, \mathfrak{h}_{P}^{(1)}\right\rangle} \mathrm{e}^{-\left\langle\mathfrak{h}_{P}^{(1)}, \mathfrak{h}_{P}^{(1)}\right\rangle} \mathrm{e}^{\left\langle\mathfrak{h}_{P}^{(1)}, \mathfrak{h}_{P}^{(0)}\right\rangle} \\
= & \prod_{j \in J}\left(\mathbf{1}+\left(\mathfrak{A} \psi_{j}\right)^{(1)} \psi_{j}^{(0)}-\left(\mathfrak{A} \psi_{j}\right)^{(1)} \psi_{j}^{(1)}+\left(\mathfrak{A} \psi_{j}\right)^{(0)} \psi_{j}^{(1)}-\left(\mathfrak{A} \psi_{j}\right)^{(0)} \psi_{j}^{(0)}\right),
\end{aligned}
$$

while, for all $j \in J$,

$$
\frac{\delta}{\delta \psi_{j}^{(1)}} \frac{\delta}{\delta\left(\mathfrak{A} \psi_{j}\right)^{(1)}}\left(\mathbf{1}+\left(\mathfrak{A} \psi_{j}\right)^{(1)} \psi_{j}^{(0)}-\left(\mathfrak{A} \psi_{j}\right)^{(1)} \psi_{j}^{(1)}+\left(\mathfrak{A} \psi_{j}\right)^{(0)} \psi_{j}^{(1)}-\left(\mathfrak{A} \psi_{j}\right)^{(0)} \psi_{j}^{(0)}\right)=-\mathbf{1}
$$

See $[\mathrm{BdSPX}]$ for more details. (Note that we use Notation 3.2.). Fix next $N \in \mathbb{N}$ and $j_{1}, \ldots, j_{N+1} \in$ $J$. Then, using Equation (55), we obtain that

$$
\begin{aligned}
& \left(\psi_{j_{1}} \wedge \cdots \wedge \psi_{j_{N}}\right) \circ_{P}\left(\mathfrak{A} \psi_{j_{N+1}}\right) \\
= & (-1)^{\frac{\operatorname{dim} \mathcal{H}}{2}} \int_{P} \mathrm{~d}\left(\mathcal{H}^{(1)}\right) \psi_{j_{1}}^{(1)} \wedge \cdots \wedge \psi_{j_{N}}^{(1)} \wedge\left(\mathfrak{A} \psi_{j_{N+1}}\right)^{(1)} \\
& \prod_{j \in J}\left(\mathbf{1}+\left(\mathfrak{A} \psi_{j}\right)^{(1)} \psi_{j}^{(0)}-\left(\mathfrak{A} \psi_{j}\right)^{(1)} \psi_{j}^{(1)}+\left(\mathfrak{A} \psi_{j}\right)^{(0)} \psi_{j}^{(1)}-\left(\mathfrak{A} \psi_{j}\right)^{(0)} \psi_{j}^{(0)}\right),
\end{aligned}
$$

\footnotetext{
${ }^{13}$ Here we use the convention $\prod_{m \in \emptyset}=1$.
} 
which, together with (56) and $|J|=\operatorname{dim}(\mathcal{H}) / 2$, implies that

$$
\begin{aligned}
& \left(\psi_{j_{1}} \wedge \cdots \wedge \psi_{j_{N}}\right) \circ_{P}\left(\mathfrak{A} \psi_{j_{N+1}}\right) \\
= & (-1)^{\left|\left\{j_{1}, \ldots, j_{N+1}\right\}\right|}\left(\prod_{j \in\left\{j_{1}, \ldots, j_{N+1}\right\}} \frac{\delta}{\delta \psi_{j}^{(1)}} \frac{\delta}{\delta\left(\mathfrak{A} \psi_{j}\right)^{(1)}}\right) \psi_{j_{1}}^{(1)} \wedge \cdots \wedge \psi_{j_{N}}^{(1)} \wedge \mathfrak{A} \psi_{j_{N+1}}^{(1)} \\
& \prod_{j \in\left\{j_{1}, \ldots, j_{N+1}\right\}}\left(1+\left(\mathfrak{A} \psi_{j}\right)^{(1)} \psi_{j}^{(0)}-\left(\mathfrak{A} \psi_{j}\right)^{(1)} \psi_{j}^{(1)}+\left(\mathfrak{A} \psi_{j}\right)^{(0)} \psi_{j}^{(1)}-\left(\mathfrak{A} \psi_{j}\right)^{(0)} \psi_{j}^{(0)}\right) .
\end{aligned}
$$

It follows that

$$
\begin{aligned}
& \left(\psi_{j_{1}} \wedge \cdots \wedge \psi_{j_{N}}\right) \circ_{P}\left(\mathfrak{A} \psi_{j_{N+1}}\right) \\
= & \psi_{j_{1}} \wedge \cdots \wedge \psi_{j_{N}} \wedge \mathfrak{A} \psi_{j_{N+1}}+\sum_{q=1}^{N}(-1)^{N-q}\left\langle\mathfrak{A} \psi_{j_{q}}, P^{\perp} \mathfrak{A} \psi_{j_{N+1}}\right\rangle_{\mathcal{H}} \prod_{j \in\left\{j_{1}, \ldots, j_{N}\right\} \backslash\left\{j_{q}\right\}} \psi_{j} .
\end{aligned}
$$

By Definition of the product $\circ_{P}$ and multilinearity of all the products appearing in the above expression, ones deduces Assertion (v) from this last equation.

It remains to prove Assertion (vi). We proceed by induction. The case $N=2$ follows from Assertion (v) and assume that Assertion (vi) holds true at some $N \in \mathbb{N} \backslash\{1\}$. Using the notation

$$
\aleph_{P}\left(\varphi_{1}, \ldots, \varphi_{m}\right) \doteq \frac{1}{m !} \sum_{\pi \in \mathcal{S}_{m}}(-1)^{\pi} \varphi_{\pi(1)} \circ_{P} \cdots \circ_{P} \varphi_{\pi(m)}
$$

for any $m \in\{1, \ldots, N+1\}$, observe that

$$
\aleph_{P}\left(\varphi_{1}, \ldots, \varphi_{N+1}\right)=\frac{1}{(N+1) !} \sum_{\pi \in \mathcal{S}_{N+1}}(-1)^{\pi} \aleph_{P}\left(\varphi_{\pi(1)}, \ldots, \varphi_{\pi(N)}\right) \circ_{P} \varphi_{\pi(N+1)}
$$

Thus, if Assertion (vi) holds true at some $N \in \mathbb{N} \backslash\{1\}$, then, for any $\varphi_{1}, \ldots, \varphi_{N+1} \in \mathcal{H}$ and permutation $\pi \in \mathcal{S}_{N+1}$,

$$
\begin{aligned}
\aleph_{P}\left(\varphi_{\pi(1)}, \ldots, \varphi_{\pi(N)}\right) \circ_{P} \varphi_{\pi(N+1)}= & \sum_{\mathcal{N} \subseteq\{1, \ldots, N\},|\mathcal{N}| \in 2 \mathbb{N}_{0}} 2^{-\frac{|\mathcal{N}|}{2}} \operatorname{Pf}\left[\left\langle\mathfrak{A} \varphi_{\pi(k)}, \frac{P^{\perp}-P}{2} \varphi_{\pi(l)}\right\rangle_{\mathcal{H}}\right]_{k, l \in \mathcal{N}} \\
& \operatorname{sign}(\mathcal{N},\{1, \ldots, N\})\left(\prod_{m \in\{1, \ldots, N\} \backslash \mathcal{N}} \varphi_{\pi(m)}\right) \circ_{P} \varphi_{\pi(N+1)}
\end{aligned}
$$

Thus, by applying Assertion (v),

$$
\begin{aligned}
& \frac{1}{(N+1) !} \sum_{\pi \in \mathcal{S}_{N+1}}(-1)^{\pi} \aleph_{P}\left(\varphi_{\pi(1)}, \ldots, \varphi_{\pi(N)}\right) \circ_{P} \varphi_{\pi(N+1)} \\
= & \mathbf{X}+\frac{1}{(N+1) !} \sum_{\pi \in \mathcal{S}_{N+1}}(-1)^{\pi} \sum_{\mathcal{N} \subseteq\{1, \ldots, N\},|\mathcal{N}| \in 2 \mathbb{N}_{0}} 2^{-\frac{|\mathcal{N}|}{2}} \operatorname{Pf}\left[\left\langle\mathfrak{A} \varphi_{\pi(k)}, \frac{P^{\perp}-P}{2} \varphi_{\pi(l)}\right\rangle_{\mathcal{H}}\right]_{k, l \in \mathcal{N}} \\
& \times \operatorname{sign}(\mathcal{N},\{1, \ldots, N+1\}) \prod_{m \in\{1, \ldots, N+1\} \backslash \mathcal{N}} \varphi_{\pi(m)},
\end{aligned}
$$


where

$$
\begin{aligned}
\mathbf{X} \doteq \frac{1}{(N+1) !} \sum_{\pi \in \mathcal{S}_{N+1}}(-1)^{\pi} \sum_{\mathcal{N} \subseteq\{1, \ldots, N\},|\mathcal{N}| \in 2 \mathbb{N}_{0}} \sum_{q \in\{1, \ldots, N\} \backslash \mathcal{N}}(-1)^{|\{1, \ldots, N\} \backslash \mathcal{N}|-|\{1, \ldots, q\} \cap\{1, \ldots, N\} \backslash \mathcal{N}|} \\
\times \operatorname{sign}(\mathcal{N},\{1, \ldots, N+1\}) 2^{-\frac{|\mathcal{N}|}{2}-1}\left\langle\mathfrak{A} \varphi_{\pi(q)}, \frac{P^{\perp}-P}{2} \varphi_{\pi(N+1)}\right\rangle_{\mathcal{H}} \\
\times \operatorname{Pf}\left[\left\langle\mathfrak{A} \varphi_{\pi(k)}, \frac{P^{\perp}-P}{2} \varphi_{\pi(l)}\right\rangle_{\mathcal{H}}\right]_{k, l \in \mathcal{N}} \prod_{m \in\{1, \ldots, N\} \backslash(\mathcal{N} \cup\{q\})} \varphi_{\pi(m)} .
\end{aligned}
$$

Now, by elementary manipulations, one gets that

$$
\begin{aligned}
\mathbf{X}= & \frac{1}{(N+1) !} \sum_{\pi \in \mathcal{S}_{N+1}}(-1)^{\pi} \sum_{\mathcal{M} \subseteq\{1, \ldots, N+1\},} \sum_{\mathcal{M} \supseteq\{N+1\},|\mathcal{M}| \in 2 \mathbb{N}_{0}} \mathbf{Y}_{\mathcal{M}, q, N} \\
& \times 2^{-\frac{|\mathcal{M}|}{2}}\left\langle\mathfrak{A} \varphi_{\pi(N+1)}, \frac{P^{\perp}-P}{2} \varphi_{\pi(q)}\right\rangle_{\mathcal{H}} \operatorname{Pf}\left[\left\langle\mathfrak{A} \varphi_{\pi(k)}, \frac{P^{\perp}-P}{2} \varphi_{\pi(l)}\right\rangle_{\mathcal{H}}\right]_{k, l \in(\mathcal{M} \backslash\{q, N+1\})} \\
& \times \prod_{m \in\{1, \ldots, N+1\} \backslash \mathcal{M}} \varphi_{\pi(m)},
\end{aligned}
$$

where, for any $N \in \mathbb{N}, \mathcal{M} \subseteq\{1, \ldots, N+1\}$ with $|\mathcal{M}| \in 2 \mathbb{N}_{0}, \mathcal{M} \supseteq\{N+1\}$, and $q \in \mathcal{M} \backslash\{N+1\}$,

$$
\begin{aligned}
\mathbf{Y}_{\mathcal{M}, q, N} \doteq(-1)^{|\{1, \ldots, N+1\} \backslash(\mathcal{M} \backslash\{q, N+1\})|-|\{1, \ldots, q\} \cap\{1, \ldots, N+1\} \backslash(\mathcal{M} \backslash\{q, N+1\})|} \\
\\
\times \operatorname{sign}((\mathcal{M} \backslash\{q, N+1\}),\{1, \ldots, N+1\}) .
\end{aligned}
$$

It is straightforward to check that

$$
\begin{aligned}
\operatorname{sign}(\mathcal{M},\{1, \ldots, N+1\})= & \operatorname{sign}(\mathcal{M} \backslash\{q, N+1\},\{1, \ldots, N+1\}) \\
& \times(-1)^{|\{1, \ldots, q\} \cap\{1, \ldots, N+1\} \backslash(\mathcal{M} \backslash\{q, N+1\})|+|\{1, \ldots, q\} \cap \mathcal{M}|+N+1},
\end{aligned}
$$

that is,

$$
\mathbf{Y}_{\mathcal{M}, q, N}=(-1)^{|\{1, \ldots, q\} \cap \mathcal{M}|} \operatorname{sign}(\mathcal{M},\{1, \ldots, N+1\}) .
$$

It follows from (58) that

$$
\begin{aligned}
\mathbf{X}=\frac{1}{(N+1) !} \sum_{\pi \in \mathcal{S}_{N+1}}(-1)^{\pi} \sum_{\mathcal{M} \subseteq\{1, \ldots, N+1\}, \mathcal{M} \supseteq\{N+1\},|\mathcal{M}| \in 2 \mathbb{N}_{0}} \operatorname{sign}(\mathcal{M},\{1, \ldots, N+1\}) \\
\times \sum_{q \in \mathcal{M} \backslash\{N+1\}}(-1)^{|\{1, \ldots, q\} \cap \mathcal{M}|} 2^{-\frac{|\mathcal{M}|}{2}}\left\langle\mathfrak{A} \varphi_{\pi(N+1)}, \frac{P^{\perp}-P}{2} \varphi_{\pi(q)}\right\rangle_{\mathcal{H}} \\
\times \operatorname{Pf}\left[\left\langle\mathfrak{A} \varphi_{\pi(k)}, \frac{P^{\perp}-P}{2} \varphi_{\pi(l)}\right\rangle_{\mathcal{H}}\right]_{k, l \in(\mathcal{M} \backslash\{q, N+1\})} \prod_{m \in\{1, \ldots, N+1\} \backslash \mathcal{M}} \varphi_{\pi(m)},
\end{aligned}
$$

which, by the well-known cofactor expansion for Pfaffians [FKT02, Proposition B.2], lead to

$$
\begin{aligned}
\mathbf{X}= & \frac{1}{(N+1) !} \sum_{\pi \in \mathcal{S}_{N+1}}(-1)^{\pi} \sum_{\mathcal{M} \subseteq\{1, \ldots, N+1\},|\mathcal{M} \cap\{N+1\}|=1,|\mathcal{M}| \in 2 \mathbb{N}_{0}} 2^{-\frac{|\mathcal{M}|}{2}} \\
& \times \operatorname{Pf}\left[\left\langle\mathfrak{A} \varphi_{\pi(k)}, \frac{P^{\perp}-P}{2} \varphi_{\pi(l)}\right\rangle_{\mathcal{H}}\right]_{k, l \in \mathcal{M}} \operatorname{sign}(\mathcal{M},\{1, \ldots, N+1\}) \prod_{m \in\{1, \ldots, N+1\} \backslash \mathcal{M}} \varphi_{\pi(m)} .
\end{aligned}
$$

By combining this with (57), we conclude that Assertion (vi) holds true for the case $N+1$, provided it holds true for $N \in \mathbb{N}$. 


\section{Remark 3.11}

By Lemma 3.10, for any basis projection $P, m, n \in \mathbb{N}_{0}$ so that $m+n \geq 1$, and all $\varphi_{1}, \ldots, \varphi_{n+m} \in \mathfrak{h}_{P}$,

$$
\left(\mathfrak{A} \varphi_{1}\right) \circ_{P} \cdots \circ_{P}\left(\mathfrak{A} \varphi_{m}\right) \circ_{P} \varphi_{m+1} \circ_{P} \cdots \circ_{P} \varphi_{m+n}=\left(\mathfrak{A} \varphi_{1}\right) \wedge \cdots \wedge\left(\mathfrak{A} \varphi_{m}\right) \wedge \varphi_{m+1} \wedge \cdots \wedge \varphi_{m+n} .
$$

If $m=0$, then there is no " $\mathfrak{A} \varphi$ " in the above equation. Mutatis mutandis for $n=0$.

\section{Remark 3.12}

For any pair of basis projections $P_{1}, P_{2} \in \mathcal{B}(\mathcal{H})$, recall the existence of a Bogoliubov transformation $U$ such that $P_{2}=U P_{1} U^{*}$, by [A68, Lemma 3.6]. Then, one deduces from Lemma $3.10(v)$ that

$$
\left(U \varphi_{1}\right) \circ_{P_{2}}\left(U \varphi_{2}\right)-\varphi_{1} \circ_{P_{1}} \varphi_{2}=\left(U \varphi_{1}\right) \wedge\left(U \varphi_{2}\right)-\varphi_{1} \wedge \varphi_{2}, \quad \varphi_{1}, \varphi_{2} \in \mathcal{H}
$$

The space $\left(\wedge^{*} \mathcal{H},+\right)$ endowed with the circle product $\circ_{P}$ is thus an (associative and distributive) algebra, like $\left(\wedge^{*} \mathcal{H},+, \wedge\right)$, for any basis projection $P$. By using the antiunitary involution $\mathfrak{A}$ on $\mathcal{H}$, we can define an involution on $\wedge^{*} \mathcal{H}$, which turns $\left(\wedge^{*} \mathcal{H},+, \circ_{P}\right)$ into a $*$-algebra:

\section{Definition 3.13 (Involution on $\wedge^{*} \mathcal{H}$ )}

The antilinear map $\xi \mapsto \xi^{*}$ from $\wedge^{*} \mathcal{H}$ to itself is uniquely defined for any basis projection $P$ by the conditions $1^{*}=1$ and

$$
\left(\varphi_{1} \circ_{P} \cdots \circ_{P} \varphi_{n}\right)^{*}=\left(\mathfrak{A} \varphi_{n}\right) \circ_{P} \cdots \circ_{P}\left(\mathfrak{A} \varphi_{1}\right), \quad n \in \mathbb{N}, \varphi_{1}, \ldots, \varphi_{n} \in \mathcal{H}
$$

The involution on $\wedge^{*} \mathcal{H}$ does not depend on the choice of the basis projection $P$ and makes the Grassmann algebra $\left(\wedge^{*} \mathcal{H},+, \wedge\right)$ a $*$-algebra:

\section{Lemma 3.14 (Grassmann algebra as *-algebra)}

$\left(\wedge^{*} \mathcal{H},+, \wedge\right)$ equipped with the involution * defined from Definition 3.13 is a *-algebra, i.e.,

$$
\left(\xi_{0} \wedge \xi_{1}\right)^{*}=\xi_{1}^{*} \wedge \xi_{0}^{*}, \quad \xi_{0}, \xi_{1} \in \wedge^{*} \mathcal{H}
$$

Proof. Fix a basis projection $P$ with range $\mathfrak{h}_{P}$. It suffices to show (59) for monomials $\xi_{0}, \xi_{1}$ in $\varphi \in \mathfrak{h}_{P}$ and $\phi^{*} \in \mathfrak{h}_{P}^{*}$, by linearity and antilinearity. This special case can be shown with straightforward computations. See for instance Remark 3.11.

For any basis projection $P$, the algebra $\left(\wedge^{*} \mathcal{H},+, \circ_{P}\right)$ endowed with this involution shares, by construction, all properties of self-dual CAR algebra except for the norm:

\section{Theorem 3.15 (From Grassmann algebra to self-dual CAR algebra)}

Given a self-dual Hilbert space $\mathcal{H}$ with even dimension $\operatorname{dim} \mathcal{H} \in 2 \mathbb{N}$ and antiunitary involution $\mathfrak{A}$, and a basis projection $P$ associated with $(\mathcal{H}, \mathfrak{A}),\left(\wedge^{*} \mathcal{H},+, \circ_{P},{ }^{*}\right)$ is a $*$-algebra generated by 1 and the family $\left\{\varphi^{*}\right\}_{\varphi \in \mathcal{H}}$ of elements satisfying Conditions (a)-(c) of Definition 2.1.

Proof. Conditions (a)-(b) of Definition 2.1 applied to the family $\left\{\varphi^{*}\right\}_{\varphi \in \mathcal{H}}$ are easily verified. Only Condition (c), that is,

$$
\varphi_{1}^{*} \circ_{P} \varphi_{2}+\varphi_{2} \circ_{P} \varphi_{1}^{*}=\left(\mathfrak{A} \varphi_{1}\right) \circ_{P} \varphi_{2}+\varphi_{2} \circ_{P}\left(\mathfrak{A} \varphi_{1}\right)=\left\langle\varphi_{1}, \varphi_{2}\right\rangle_{\mathcal{H}} \mathbf{1}, \quad \varphi_{1}, \varphi_{2} \in \mathcal{H},
$$

has to be checked: Condition (c) results from Lemma 3.10, in particular Assertion (v), together with properties of the antiunitary involution $\mathfrak{A}$, Definition 3.13 and elementary computations.

For two different basis projections $P_{1}, P_{2} \in \mathcal{B}(\mathcal{H}),\left(\wedge^{*} \mathcal{H},+, \circ_{P_{1}},{ }^{*}\right)$ and $\left(\wedge^{*} \mathcal{H},+, \circ_{P_{2}},{ }^{*}\right)$ differ as algebras because their respective circle products do not coincide, in general. See Remark 3.12. They are, nevertheless, $*$-isomorphic to each other. In the same way, there is a canonical $*$-isomorphism between a self-dual CAR algebra constructed from $(\mathcal{H}, \mathfrak{A})$ and $\wedge^{*} \mathcal{H}$ : 
Definition 3.16 (Canonical isomorphism of $*$-algebra)

For any basis projection $P$, we define the canonical isomorphism

$$
\varkappa_{P}:\left(\operatorname{sCAR}(\mathcal{H}, \mathfrak{A}),+, \cdot{ }^{*}\right) \rightarrow\left(\wedge^{*} \mathcal{H},+, \circ_{P},{ }^{*}\right)
$$

via the conditions $\varkappa_{P}(z \mathbf{1})=z \mathbf{1}$ and $\varkappa_{P}(\mathrm{~B}(\varphi))=\varphi^{*}$ for all $\varphi \in \mathcal{H}$.

Bilinear elements of self-dual CAR algebra (Definition 2.5) are mapped via $\varkappa_{P}$ (up to some constant) to bilinear elements of Grassmann algebra, as stated in Definition 3.5: By (17), Lemma 3.10 (v) and Definition 3.16, for any basis projection $P$,

$$
\varkappa_{P}(\langle\mathrm{~B}, H \mathrm{~B}\rangle)=\langle\mathcal{H}, H \mathcal{H}\rangle+\operatorname{Tr}_{\mathcal{H}}\left(P^{\perp} H P^{\perp}\right) 1, \quad H \in \mathcal{B}(\mathcal{H}) .
$$

Compare with Equations (24) and (120).

To make $\left(\wedge^{*} \mathcal{H},+, \circ_{P},{ }^{*}\right)$ a self-dual CAR $\left(C^{*}-\right)$ algebra for a basis projection $P$, it suffices to equip this $*$-algebra with the following norm:

$$
\|\xi\|_{\wedge^{*} \mathcal{H}} \doteq\left\|\varkappa_{P}^{-1}(\xi)\right\|_{\operatorname{sCAR}(\mathcal{H}, \mathfrak{A})}, \quad \xi \in \wedge^{*} \mathcal{H} .
$$

See Definition 2.1 and Theorem 3.15. In this case, $\varkappa_{P}$ is an isometry.

\section{Remark 3.17}

For any basis projection $P$ with range $\mathfrak{h}_{P}, \wedge^{*} \mathcal{H}$ can be equipped with a scalar product and then seen as the fermionic Fock space $\mathcal{F}_{\mathcal{H}}$, as explained in Section 6.4. By finite dimensionality of $\mathcal{H}$, the norm (61) is equivalent, albeit different, to the one coming from the scalar product. In fact, $\wedge^{*} \mathcal{H}$ can be viewed as the $C^{*}$-algebra $\mathcal{B}\left(\mathcal{F}_{\mathfrak{h}_{P}}\right)$, the norm (61) being the operator norm.

\section{Generating Functions as Berezin Integrals}

First, we use a Chernoff product formula for the exponentials $\mathrm{e}^{-\beta H}$ and $\mathrm{e}^{s K}$, see Equation (2). Using the tracial state formula (Theorem 4.1) we then deduce a representation of generating functions as Gaussian Berezin integrals (Definition 3.6). More precisely, we prove Equation (6). This latter equation refers to a kind of fermionic path integral for generating functions, which is one of the objectives of the paper.

Note that in all the sections, $P$ is always a basis projection (Definition 2.4), which determines the product $\circ_{P}$ of the self-dual CAR algebra $\left(\wedge^{*} \mathcal{H},+, \circ_{P},{ }^{*}\right)$.

\subsection{The Berezin Integral Representation of the Tracial State}

Through the isomorphism $\varkappa_{P}$, defined for any basis projection $P$ by Definition 3.16 , the tracial state $\operatorname{tr} \in \operatorname{sCAR}(\mathcal{H}, \mathfrak{A})^{*}$ (Definition 2.9) can be represented in terms of Berezin integrals. To explain this, we introduce the following definitions:

(i): For any basis projection $P$ and integers $n \in \mathbb{N}_{0}$ and $k \in\{0, \ldots, n\}$, we define the map

$$
\varkappa_{P}^{(k)} \doteq \varkappa_{(0,0)}^{(k, k)} \circ \varkappa_{P}
$$

from $\operatorname{sCAR}(\mathcal{H}, \mathfrak{A})$ to the $k$-copy $\wedge^{*} \mathcal{H}^{(k)}$ of $\wedge^{*} \mathcal{H}$. Note that $\varkappa_{P}^{(0)}=\varkappa_{P}$, see (43) and Definition 3.16. (ii): Using Notation 1.3 (ii), we define the finite-dimensional Hilbert spaces

$$
\mathfrak{H}^{(n)} \doteq \bigoplus_{k=0}^{n-1} \mathcal{H}^{(k)}, \quad n \in \mathbb{N}
$$


The corresponding scalar product is the usual one for direct sums of Hilbert spaces. In particular, for any orthonormal basis $\left\{\psi_{i}\right\}_{i \in I}$ of $\mathcal{H}$, the union $\cup_{k=0}^{n-1}\left\{\psi_{i}^{(k)}\right\}_{i \in I}$ is an orthonormal basis of $\mathfrak{H}^{(n)}$.

(iii): For any $n \in \mathbb{N}$, a map $A$ from $\mathcal{H}$ to itself, like the antiunitary involution $\mathfrak{A}$, is canonically extended to a map $\hat{A}$ from $\mathfrak{H}^{(n)}$ to itself by the conditions

$$
\hat{A} \varphi^{(k)} \doteq(A \varphi)^{(k)}, \quad k \in\{0, \ldots, n-1\}
$$

Notice that $\hat{\mathfrak{A}}$ is an antiunitary involution, and any basis projection $P$ associated with $(\mathcal{H}, \mathfrak{A})$ yields a basis projection $\hat{P}$ associated with $\left(\mathfrak{H}^{(n)}, \hat{\mathfrak{A}}\right)$ for any $n \in \mathbb{N}$.

(iv): For any $n \in \mathbb{N}$, we introduce the linear map $\partial \in \mathcal{B}\left(\mathfrak{H}^{(n)}\right)$ defined by the identities

$$
\partial \varphi^{(0)}=\varphi^{(0)}+\varphi^{(n-1)} \quad \text { and } \quad \partial \varphi^{(k)}=\varphi^{(k)}-\varphi^{(k-1)}
$$

for all $\varphi \in \mathcal{H}$ and $k \in\{1, \ldots, n-1\}$. Note that its adjoint $\partial^{*}$ satisfies

$$
\partial^{*} \varphi^{(n-1)}=\varphi^{(0)}+\varphi^{(n-1)} \quad \text { and } \quad \partial^{*} \varphi^{(k)}=\varphi^{(k)}-\varphi^{(k+1)}
$$

for all $\varphi \in \mathcal{H}$ and $k \in\{0, \ldots, n-2\}$. The operator $\partial$ is normal but not self-adjoint.

(v): For every $n \in \mathbb{N}$ and any basis projection $P$ with range $\mathfrak{h}_{P}$, we then define from (64) and (65) the operator

$$
\boldsymbol{\partial}_{P} \doteq \hat{P} \partial \hat{P}-\hat{\mathfrak{A}} \hat{P} \partial^{*} \hat{P} \hat{\mathfrak{A}} \in \mathcal{B}\left(\mathfrak{H}^{(n)}\right) .
$$

It is a self-dual operator on $\left(\mathfrak{H}^{(n)}, \hat{\mathfrak{A}}\right)$ because $\partial_{P}^{*}=-\hat{\mathfrak{A}} \partial_{P} \hat{\mathfrak{A}}$. See Definitions 2.4 and 2.7 .

Then, we get the well-known tracial state formula in the context of Grassmann algebra:

\section{Theorem 4.1 (Tracial state formula)}

Fix a basis projection $P$. Then, for all $n \in \mathbb{N}$ and $A_{0}, \ldots, A_{n-1} \in \operatorname{sCAR}(\mathcal{H}, \mathfrak{A})$,

$$
\operatorname{tr}\left(A_{0} \cdots A_{n-1}\right) \mathbf{I}=2^{-\frac{\operatorname{dim} \mathcal{H}}{2}} \int_{P} \mathrm{~d}\left(\mathfrak{H}^{(n)}\right) \mathrm{e}^{\frac{1}{2}\left\langle\mathfrak{H}^{(n)}, \boldsymbol{\partial}_{P} \mathfrak{H}^{(n)}\right\rangle}\left(\prod_{k=0}^{n-1} \varkappa_{P}^{(k)}\left(A_{k}\right)\right) .
$$

Proof. Observe that Remark 6.4 holds true for $\mathfrak{h}=\mathfrak{h}_{P}$ and any basis projection $P$. Then, by using linearity properties and explicit computations on arbitrary, normally-ordered monomials, we prove that

$$
\operatorname{tr}(A) \mathbf{1}=2^{-\frac{\operatorname{dim} \mathcal{H}}{2}} \int_{P} \mathrm{~d}(\mathcal{H}) \varkappa_{P}(A) \mathrm{e}^{2\left\langle\mathfrak{h}_{P}, \mathfrak{h}_{P}\right\rangle}, \quad A \in \operatorname{sCAR}(\mathcal{H}, \mathfrak{A})
$$

Next, we use the equality

$$
\varkappa_{P}\left(A_{0} \cdots A_{n-1}\right)=\varkappa_{P}\left(A_{0}\right) \circ_{P} \cdots \circ_{P} \varkappa_{P}\left(A_{n-1}\right),
$$

together with Lemma 3.10 (i) and a renumbering, to get the assertion. Note that

$$
\int_{P} \mathrm{~d}\left(\mathfrak{H}^{(n)}\right)=\prod_{k=0}^{n-1} \int_{P} \mathrm{~d}\left(\mathcal{H}^{(k)}\right), \quad n \in \mathbb{N}
$$

by Definition 3.3, while, for any basis projection $P$,

$$
\frac{1}{2}\left\langle\mathfrak{H}^{(n)}, \boldsymbol{\partial}_{P} \mathfrak{H}^{(n)}\right\rangle=\left\langle\mathfrak{h}_{P}^{(0)}, \mathfrak{h}_{P}^{(0)}\right\rangle+\left\langle\mathfrak{h}_{P}^{(0)}, \mathfrak{h}_{P}^{(n-1)}\right\rangle+\sum_{k=1}^{n-1}\left(\left\langle\mathfrak{h}_{P}^{(k)}, \mathfrak{h}_{P}^{(k)}\right\rangle-\left\langle\mathfrak{h}_{P}^{(k)}, \mathfrak{h}_{P}^{(k-1)}\right\rangle\right)
$$

by Definition 3.5, Equations (64) and (66). For more details we recommend [BdSPX]. 


\subsection{Chernoff Product Formula}

We now define a Chernoff product approximation in self-dual algebra as follows:

\section{Definition 4.2 (A Chernoff product approximation)}

Fix a basis projection $P$. For every element $A \in \operatorname{sCAR}(\mathcal{H}, \mathfrak{A})$ and all $n \in \mathbb{N}$,

$$
X_{A}^{(n)} \doteq\left[\varkappa_{P}^{-1}\left(\exp \left(\frac{1}{n} \varkappa_{P}(A)\right)\right)\right]^{n} \in \operatorname{sCAR}(\mathcal{H}, \mathfrak{A})
$$

where we recall that $\varkappa_{P}$ is the canonical isomorphism of $*$-algebras from Definition 3.16, while $\exp (\xi)$ is defined by $(38)^{14}$ for all $\xi \in \wedge^{*} \mathcal{H}$.

In the limit $n \rightarrow \infty, X_{A}^{(n)}$ gives the usual exponential of $A$ :

\section{Theorem 4.3 (Chernoff product formula)}

For any basis projection $P$ and $A \in \operatorname{sCAR}(\mathcal{H}, \mathfrak{A})$,

$$
\left(X_{A}^{(n)}\right)^{*}=X_{A^{*}}^{(n)} \quad \text { and } \quad \lim _{n \rightarrow \infty} X_{A}^{(n)}=\exp (A) .
$$

This convergence is uniform on bounded subsets of the $C^{*}$-algebra $\operatorname{sCAR}(\mathcal{H}, \mathfrak{A})$.

Proof. Since

$$
\varkappa_{P}^{-1}\left(\exp \left(\varkappa_{P}(0)\right)\right)=\mathbf{1} \in \operatorname{sCAR}(\mathcal{H}, \mathfrak{A}) \quad \text { and } \quad \partial_{s}\left\{\varkappa_{P}^{-1}\left(\exp \left(s \varkappa_{P}(A)\right)\right)\right\}_{s=0}=A \text {, }
$$

the assertion is a usual Chernoff product formula. See, e.g., [EN00, 5.2 Theorem]. Although general results proving the Chernoff product formula are available, we provide here an explicit proof to extract properties that are particular to our choice of the Chernoff product approximation. (38),

By finite dimensionality of $\mathcal{H}$, the involution of $\wedge^{*} \mathcal{H}$ is continuous. By Lemma 3.14 and Equation

$$
[\exp (\xi)]^{*}=\exp \left(\xi^{*}\right), \quad \xi \in \wedge^{*} \mathcal{H}
$$

Since the map $\varkappa_{P}$ is a $*$-isomorphism (Definition 3.16), for every $n \in \mathbb{N}$ it follows that $X_{A}^{(n) *}=X_{A^{*}}^{(n)}$ for any $A \in \operatorname{sCAR}(\mathcal{H}, \mathfrak{A})$.

For every $n \in \mathbb{N}$ and $A \in \operatorname{sCAR}(\mathcal{H}, \mathfrak{A})$ observe next that

$$
X_{A}^{(n)}=\left(1+\frac{A}{n}\right)^{n}+\sum_{k=1}^{n}\left(\begin{array}{l}
n \\
k
\end{array}\right) \tilde{X}_{A}^{k}\left(1+\frac{1}{n} A\right)^{n-k}
$$

where

$$
\tilde{X}_{A} \doteq \sum_{m=2}^{\infty} \frac{1}{m !}\left(\frac{1}{n}\right)^{m} \varkappa_{P}^{-1}\left(\varkappa_{P}(A)^{m}\right) .
$$

So, it suffices to show that the last term of (68) converges to zero when $n \rightarrow \infty$ to prove the Chernoff product formula. By finite dimensionality of $\mathcal{H}$, note that the linear map $\varkappa_{P}$ from $\operatorname{sCAR}(\mathcal{H}, \mathfrak{A})$ to

\footnotetext{
${ }^{14}$ In other words, we use the exterior product $\wedge$ (and not the circle product from Definition 3.9) of the Grassmann algebra $\wedge^{*} \mathcal{H}$ (see (37)) to define the exponential.
} 
$\wedge^{*} \mathcal{H}$ is continuous. Moreover, its inverse $\varkappa_{P}^{-1}$ is also continuous, by Definition (61). From (39), we obtain that

$$
\begin{aligned}
\left\|\tilde{X}_{A}\right\|_{\operatorname{SCAR}(\mathcal{H}, \mathfrak{A})} & \leq \sum_{m=2}^{\infty} \frac{1}{m !}\left(\frac{1}{n}\right)^{m}\left\|\varkappa_{P}(A)^{m}\right\|_{\wedge^{* \mathcal{H}}} \\
& \leq \frac{1}{n^{2}} D_{\varkappa_{P}(A)}^{2} \sum_{m=2}^{\infty} \frac{1}{m !}\left(\frac{1}{n}\right)^{m-2} D_{\varkappa_{P}(A)}^{m-2} \\
& \leq \frac{1}{n^{2}} D_{\varkappa_{P}(A)}^{2} \exp \left(\frac{1}{n} D_{\varkappa_{P}(A)}\right) .
\end{aligned}
$$

Now, by using the definition

$$
D_{A} \doteq \max \left\{D_{\varkappa_{P}(A)} \mathrm{e}^{D_{\varkappa_{P}(A)}},\|A\|_{\mathrm{sCAR}(\mathcal{H}, \mathfrak{R})}\right\},
$$

we infer from (69) that

$$
\begin{aligned}
\left\|\sum_{k=1}^{n}\left(\begin{array}{l}
n \\
k
\end{array}\right) \tilde{X}_{A}^{k}\left(1+\frac{1}{n} A\right)^{n-k}\right\|_{\operatorname{SCAR}(\mathcal{H}, \mathfrak{A})} & \leq \sum_{k=1}^{n}\left(\begin{array}{l}
n \\
k
\end{array}\right)\left(\frac{D_{A}^{2}}{n^{2}}\right)^{k}\left(1+\frac{D_{A}}{n}\right)^{n-k} \\
& =\left(1+\frac{D_{A}}{n}+\frac{D_{A}^{2}}{n^{2}}\right)^{n}-\left(1+\frac{D_{A}}{n}\right)^{n} \\
& \leq\left(1+\frac{D_{A}}{n}\right)^{n}\left(\left(1+\frac{D_{A}^{2}}{n^{2}}\right)^{n}-1\right) \\
& \leq \mathrm{e}^{D_{A}}\left(\mathrm{e}^{\frac{D_{A}^{2}}{n}}-1\right) .
\end{aligned}
$$

By finite dimensionality of $\mathcal{H}$, for any bounded subset $\mathfrak{B}$ of $\operatorname{sCAR}(\mathcal{H}, \mathfrak{A})$,

$$
\sup _{A \in \mathfrak{B}} D_{A}<\infty \quad \text { and } \quad \lim _{n \rightarrow \infty} \sup _{A \in \mathfrak{B}}\left\|\left(1+\frac{A}{n}\right)^{n}-\exp (A)\right\|_{\operatorname{SCAR}(\mathcal{H}, \mathfrak{A})}=0 .
$$

As a consequence, by (68) and (70), the convergence, as $n \rightarrow \infty$, of $X_{A}^{(n)}$ to $\exp (A)$ is uniform on bounded subsets of $\operatorname{sCAR}(\mathcal{H}, \mathfrak{A})$.

\section{Remark 4.4 (Self-adjoint case)}

If $\mathfrak{B} \subset \operatorname{sCAR}(\mathcal{H}, \mathfrak{A})$ is a bounded subset of self-adjoint elements, then there is $N_{\mathfrak{B}} \in \mathbb{N}$ such that, for all $n \geq N_{\mathfrak{B}}, s \in \mathbb{R}$ and $A \in \mathfrak{B}$, the element $\left(X_{A}^{(n)}\right)^{s}$ is well-defined and self-adjoint, by Theorem 4.3 and the spectral theorem. Like in Theorem 4.3, it tends to $\exp (s A)$ in the limit $n \rightarrow \infty$. Moreover, in this case, again by the spectral theorem, the family of maps $s \mapsto\left(X_{A}^{(n)}\right)^{s}, n \geq N_{\mathfrak{B}}, A \in \mathfrak{B}$, is equicontinuous.

If one applies the above Chernoff product formula to bilinear elements of self-dual CAR algebra (Definition 2.5) then one gets an expression similar to a Bernoulli or Euler limit: Since, by Definition 3.5, for any $H \in \mathcal{B}(\mathcal{H})$,

$$
\mathrm{e}^{\langle\mathcal{H}, H \mathcal{H}\rangle}=\prod_{i, j \in I} \mathrm{e}^{\left\langle\psi_{i}, H \psi_{j}\right\rangle_{\mathcal{H}}\left(\mathfrak{A} \psi_{j}\right) \wedge \psi_{i}}=\prod_{i, j \in I}\left(\mathbf{1}+\left\langle\psi_{i}, H \psi_{j}\right\rangle_{\mathcal{H}}\left(\mathfrak{A} \psi_{j}\right) \wedge \psi_{i}\right)
$$

for any basis projection $P$ and operator $H \in \mathcal{B}(\mathcal{H})$, by (60),

$$
X_{\langle\mathrm{B}, H \mathrm{~B}\rangle}^{(n)}=\mathrm{e}^{\operatorname{Tr}_{\mathcal{H}}\left(P^{\perp} H P^{\perp}\right)}\left[\varkappa_{P}^{-1}\left(\prod_{i, j \in I}\left(1+\frac{1}{n}\left\langle\psi_{i}, H \psi_{j}\right\rangle_{\mathcal{H}} \psi_{j}^{*} \wedge \psi_{i}\right)\right)\right]^{n}, \quad n \in \mathbb{N},
$$


where we recall that $\varphi^{*} \doteq \mathfrak{A} \varphi$, by Definition 3.13. Because of Theorem 4.3, $X_{\langle\mathrm{B}, H \mathrm{~B}\rangle}^{(n)}$ converges to $\exp (\langle\mathrm{B}, H \mathrm{~B}\rangle)$ in the limit $n \rightarrow \infty$.

When $H$ is a self-dual Hamiltonian on $(\mathcal{H}, \mathfrak{A})$ (Definition 2.7), there is a basis projection $P$ diagonalizing $H$ (see (23)), which implies that the Chernoff product approximation (72) has an explicit formulation in terms of the generators $\{\mathrm{B}(\varphi)\}_{\varphi \in \mathcal{H}}$ of $\operatorname{sCAR}(\mathcal{H}, \mathfrak{A})$ :

\section{Lemma 4.5 (Chernoff product approximation and bilinear elements)}

Let $H$ be a self-dual Hamiltonian on $(\mathcal{H}, \mathfrak{A})$ and take a basis projection $P$ diagonalizing $H$. Let $\left\{\psi_{j}\right\}_{j \in J} \subset \mathfrak{h}_{P}$ be any orthonormal basis of eigenvectors of $h \doteq 2 P H P \in \mathcal{B}\left(\mathfrak{h}_{P}\right)$. Then, for all $n \in \mathbb{N}$ with $n>2\|H\|_{\mathcal{B}(\mathcal{H})}$ and any $s \in \mathbb{R}, X_{\langle\mathrm{B}, H \mathrm{~B}\rangle}^{(n)}>0$ and

$$
\left(X_{\langle\mathrm{B}, H \mathrm{~B}\rangle}^{(n)}\right)^{s}=\mathrm{e}^{s \operatorname{Tr}_{\mathcal{H}}\left(P^{\perp} H P^{\perp}\right)} \prod_{j \in J}\left(1+\frac{2}{n}\left\langle\psi_{j}, H \psi_{j}\right\rangle_{\mathcal{H}} \mathrm{B}\left(\psi_{j}\right) \mathrm{B}\left(\psi_{j}\right)^{*}\right)^{s n} .
$$

Proof. Under the assumptions of the lemma, the Chernoff product approximations (72) are now equal to

$$
X_{\langle\mathrm{B}, H \mathrm{~B}\rangle}^{(n)}=\mathrm{e}^{\operatorname{Tr} \mathcal{H}\left(P^{\perp} H P^{\perp}\right)}\left[\varkappa_{P}^{-1}\left(\prod_{j \in J}\left(1+\frac{2}{n}\left\langle\psi_{j}, H \psi_{j}\right\rangle_{\mathcal{H}} \psi_{j}^{*} \wedge \psi_{j}\right)\right)\right]^{n}
$$

for all $n \in \mathbb{N}$. In particular, by Lemma 3.10 (iv)-(v) and Definition 3.16,

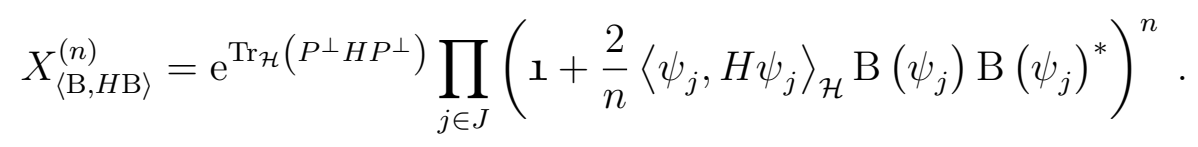

Obviously,

$$
\sup _{j \in J}\left|\left\langle\psi_{j}, H \psi_{j}\right\rangle_{\mathcal{H}}\right| \leq\|H\|_{\mathcal{B}(\mathcal{H})} .
$$

Therefore, by Definition 2.1 (c), Remark 2.2, and Equation (73), for any integer $n>2\|H\|_{\mathcal{B}(\mathcal{H})}$, the Chernoff product approximation $X_{\langle\mathrm{B}, H \mathrm{~B}\rangle}^{(n)}$ is the product of commuting and strictly positive elements

$$
\mathbf{1}+\frac{2}{n}\left\langle\psi_{j}, H \psi_{j}\right\rangle_{\mathcal{H}} \mathrm{B}\left(\psi_{j}\right) \mathrm{B}\left(\psi_{j}\right)^{*}, \quad j \in J
$$

The assertion then follows.

\subsection{Feynman-Kac-like Formula for Generating Functions}

We are now in a position to derive a Feynman-Kac-like formula for terms of the form (2), that is,

$$
\operatorname{tr}\left(\mathrm{e}^{-\beta \mathbf{H}} \mathrm{e}^{s K}\right), \quad \beta \in \mathbb{R}^{+}, s \in \mathbb{R} \quad \text { and } \quad \mathbf{H}=\mathbf{H}^{*}, K=K^{*} \in \operatorname{sCAR}(\mathcal{H}, \mathfrak{A}),
$$

with the tracial state $\operatorname{tr} \in \operatorname{sCAR}(\mathcal{H}, \mathfrak{A})^{*}$ of Definition 2.9. They are related to logarithmic moment generating functions associated with thermodynamic sequences at equilibrium. See Equations (6)-(7).

We restrict our analysis to even elements, that is, elements $A \in \operatorname{sCAR}(\mathcal{H}, \mathfrak{A})$ satisfying the property $\boldsymbol{\chi}_{-\mathbf{1}_{\mathcal{H}}}(A)=A$, see (15). Then, the Feynman-Kac-like formula we are seeking is a straightforward consequence of the Chernoff product formula (Theorem 4.3) and the tracial state formula (Theorem 4.1): 


\section{Theorem 4.6 (Feynman-Kac-like formula for the tracial state)}

Let $\mathbf{H}, K \in \operatorname{sCAR}(\mathcal{H}, \mathfrak{A})$ be two even elements, $K$ being also self-adjoint. Then, for all $\beta \in \mathbb{R}^{+}$, $s \in \mathbb{R}$ and any basis projection $P$,

$$
\begin{aligned}
\operatorname{tr}\left(\mathrm{e}^{-\beta \mathbf{H}} \mathrm{e}^{s K}\right) \mathbf{l}=2^{-\frac{\operatorname{dim} \mathcal{H}}{2}} \lim _{n \rightarrow \infty} \int_{P} \mathrm{~d}\left(\mathfrak{H}^{\left(n_{\beta}\right)}\right) \mathrm{e}^{\frac{1}{2}\left\langle\mathfrak{H}^{\left(n_{\beta}\right)}, \boldsymbol{\partial}_{P} \mathfrak{H}^{\left(n_{\beta}\right)}\right\rangle} & \\
& \wedge \exp \left(\frac{\beta s}{n} \sum_{k=n}^{n_{\beta}-1} \varkappa_{P}^{(k)}(K)-\frac{\beta}{n} \sum_{k=0}^{n-1} \varkappa_{P}^{(k)}(\mathbf{H})\right)
\end{aligned}
$$

with $\varkappa_{P}^{(k)}, \mathfrak{H}^{\left(n_{\beta}\right)}$ and $\partial_{P}$ respectively defined by (62), (63) and (66). Here, $n_{\beta} \doteq n+\lfloor n / \beta\rfloor$, with $\lfloor x\rfloor$ being the largest natural number smaller than $x \in \mathbb{R}^{+}$.

Proof. Fix $\beta \in \mathbb{R}^{+}, s \in \mathbb{R}$, two elements $\mathbf{H}, K=K^{*} \in \operatorname{sCAR}(\mathcal{H}, \mathfrak{A})$ and any basis projection $P$. We then infer from Theorem 4.3 and Remark 4.4 that

$$
\operatorname{tr}\left(\mathrm{e}^{-\beta \mathbf{H}} \mathrm{e}^{s K}\right)=\lim _{n \rightarrow \infty} \operatorname{tr}\left(X_{-\beta \mathbf{H}}^{(n)}\left(X_{s \beta K}^{(n)}\right)^{n^{-1}\lfloor n / \beta\rfloor}\right),
$$

which, combined with (62), Theorem 4.1 and Definition 4.2, in turn implies that

$$
\begin{aligned}
\operatorname{tr}\left(\mathrm{e}^{-\beta \mathbf{H}} \mathrm{e}^{s K}\right)=2^{-\frac{\operatorname{dim} \mathcal{H}}{2}} \lim _{n \rightarrow \infty} \int_{P} \mathrm{~d}\left(\mathfrak{H}^{\left(n_{\beta}\right)}\right) \mathrm{e}^{\frac{1}{2}\left\langle\mathfrak{H}^{\left(n_{\beta}\right)}, \boldsymbol{\partial}_{P} \mathfrak{H}^{\left(n_{\beta}\right)}\right\rangle}\left[\prod_{k=0}^{n-1} \exp \left(-\frac{\beta}{n} \varkappa_{P}^{(k)}(\mathbf{H})\right)\right] \\
\wedge \prod_{k=n}^{n_{\beta}-1} \exp \left(\frac{\beta s}{n} \varkappa_{P}^{(k)}(K)\right) .
\end{aligned}
$$

Now, if $\mathbf{H}$ and $K$ are even, then this last equation implies the assertion. Observe that the products involved in the right-hand side of Equation (74) all refer to the exterior product $\wedge$ of $\wedge^{*} \mathcal{H}$. Thus, this space is viewed as Grassmann algebra (Definition 3.1).

\section{Remark 4.7 (Non-even and non-self-adjoint case)}

Equation (74) gives a Feynman-Kac-like formula for traces of the form (2) for all $\mathbf{H}, K=K^{*} \in$ $\operatorname{sCAR}(\mathcal{H}, \mathfrak{A})$. We focus our study on the cases for which $\mathbf{H}$ and $K$ are even, which is the typical case for fermionic models in theoretical physics. Note also that the self-adjointness of $K$ could be relaxed. We omit the details.

We focus on bilinear elements (Definition 2.5) perturbed by an even, self-adjoint element $W=$ $W^{*} \in \operatorname{sCAR}(\mathcal{H}, \mathfrak{A})$, that is, fermionic Hamiltonians of the form

$$
\mathbf{H}=-\frac{1}{2}\langle\mathrm{~B}, H \mathrm{~B}\rangle+W, \quad H=H^{*} \in \mathcal{B}(\mathcal{H}) .
$$

In this case, the Feynman-Kac-like formula for traces can be written in terms of a limit of a Gaussian Berezin integral (Definition 3.6):

\section{Corollary 4.8 (Feynman-Kac-like formula for generating functions)}

Let $H \in \mathcal{B}(\mathcal{H})$ be any self-dual Hamiltonian, and let $W, K \in \operatorname{sCAR}(\mathcal{H}, \mathfrak{A})$ be even elements, with $K$ being self-adjoint. Then, for all $\beta \in \mathbb{R}^{+}, s \in \mathbb{R}$ and any basis projection $P$ diagonalizing $H$,

$$
\frac{\operatorname{tr}\left(\mathrm{e}^{\beta\left(\frac{1}{2}\langle\mathrm{~B}, H \mathrm{~B}\rangle-W\right)} \mathrm{e}^{s K}\right)}{\operatorname{tr}\left(\mathrm{e}^{\frac{\beta}{2}\langle\mathrm{~B}, H \mathrm{~B}\rangle}\right)} \mathbf{1}=\lim _{n \rightarrow \infty} \int \mathrm{d} \mu_{C_{H, P}^{(n)}}\left(\mathfrak{H}^{\left(n_{\beta}\right)}\right) \exp \left(\frac{\beta s}{n} \sum_{k=n}^{n_{\beta}-1} \varkappa_{P}^{(k)}(K)-\frac{\beta}{n} \sum_{k=0}^{n-1} \varkappa_{P}^{(k)}(W)\right) .
$$


Here, for any integer $n>\beta\|H\|_{\mathcal{B}(\mathcal{H})}$,

$$
C_{H, P}^{(n)} \doteq\left(\boldsymbol{\partial}_{P}+\frac{\beta}{n} \mathbf{1}[k<n] \hat{H}\right)^{-1} \in \mathcal{B}\left(\mathfrak{H}^{\left(n_{\beta}\right)}\right)
$$

with $\hat{H}$ and $\partial_{P}$ respectively defined by (64) and (66), while $\mathbf{1}[k<n]$ is the orthogonal projection on the subspace $\mathfrak{H}^{(n)} \subset \mathfrak{H}^{\left(n_{\beta}\right)}$ (63). Recall that $n_{\beta} \doteq n+\lfloor n / \beta\rfloor$.

Proof. Fix all the parameters of the corollary. On the one hand, we deduce from Lemmata 3.10 (iv)-(v) and 4.5, together with Definition 3.16 and elementary computations like (46) and (71), that, for all $n \in \mathbb{N}$ such that $n>\beta\|H\|_{\mathcal{B}(\mathcal{H})}$,

$$
\mathrm{e}^{\frac{\beta}{2 n}\left\langle\mathfrak{H}^{(n)}, \hat{H} \mathfrak{H}^{(n)}\right\rangle}=\mathrm{e}^{-\frac{\beta}{2} \operatorname{Tr}_{\mathcal{H}}\left(P^{\perp} H P^{\perp}\right)} \prod_{k=0}^{n-1} \varkappa_{P}^{(k)}\left(\left[X_{\beta\langle\mathrm{B}, H \mathrm{~B}\rangle / 2}^{(n)}\right]^{\frac{1}{n}}\right) .
$$

The product here refers to the exterior one, $\wedge$ (Notation 3.2). On the other hand, we deduce from (47) that

$$
\operatorname{det}\left[\hat{P} B_{H, P}^{(n)} \hat{P}\right] \mathbf{I}=\int_{\hat{P}} \mathrm{~d}\left(\mathfrak{H}^{\left(n_{\beta}\right)}\right) \mathrm{e}^{\frac{1}{2}\left\langle\mathfrak{H}^{\left(n_{\beta}\right)}, \boldsymbol{\partial}_{P} \mathfrak{H}^{\left(n_{\beta}\right)}\right\rangle} \mathrm{e}^{\frac{\beta}{2 n}\left\langle\mathfrak{H}^{(n)}, \hat{H} \mathfrak{H}^{(n)}\right\rangle}, \quad n \in \mathbb{N},
$$

with

$$
B_{H, P}^{(n)} \doteq \frac{1}{2}\left(\boldsymbol{\partial}_{P}+\frac{\beta}{n} \mathbf{1}[k<n] \hat{H}\right) \in \mathcal{B}\left(\mathfrak{H}^{\left(n_{\beta}\right)}\right) .
$$

Therefore, we infer from (77) and (78), together with Theorem 4.1, that

$$
\operatorname{det}\left[\hat{P} B_{H, P}^{(n)} \hat{P}\right]=2^{\frac{\operatorname{dim} \mathcal{H}}{2}} \mathrm{e}^{-\frac{\beta}{2} \operatorname{Tr} \mathcal{H}}\left(P^{\perp} H P^{\perp}\right) \operatorname{tr}\left(X_{\beta\langle\mathrm{B}, H \mathrm{~B}\rangle / 2}^{(n)}\right)>0
$$

for any integer $n>\beta\|H\|_{\mathcal{B}(\mathcal{H})}$, since the tracial state tr is faithful and $X_{\beta\langle\mathrm{B}, H \mathrm{~B}\rangle / 2}^{(n)}>0$ (Lemma 4.5). In particular, for $n>\beta\|H\|_{\mathcal{B}(\mathcal{H})}$, the operator $\hat{P} B_{H, P}^{(n)} \hat{P}$ is invertible in the range $\mathfrak{h}_{\hat{P}}$. Note that $B_{H, P}^{(n)}$ is self-dual on $\left(\mathfrak{H}^{\left(n_{\beta}\right)}, \hat{\mathfrak{A}}\right)$ because $H \in \mathcal{B}(\mathcal{H})$ is a self-dual operator by assumption. Moreover, if $P$ is a basis projection diagonalizing $H$, then $\hat{P}$ is a basis projection diagonalizing $B_{H, P}^{(n)}$. Hence, $B_{H, P}^{(n)}$ is invertible for $n>\beta\|H\|_{\mathcal{B}(\mathcal{H})}$ and the covariance $C_{H, P}^{(n)}$ is, in this case, well-defined.

Now, we infer from Theorem 4.6 and Equation (60) that

$$
\begin{aligned}
& 2^{\frac{\operatorname{dim} \mathcal{H}}{2}} \mathrm{e}^{-\frac{\beta}{2} \operatorname{Tr} \mathcal{H}\left(P^{\perp} H P^{\perp}\right)} \operatorname{tr}\left(\mathrm{e}^{\beta\left(\frac{1}{2}\langle\mathrm{~B}, H \mathrm{~B}\rangle-W\right)} \mathrm{e}^{s K}\right) \mathbf{l} \\
& =\lim _{n \rightarrow \infty} \int_{\hat{P}} \mathrm{~d}\left(\mathfrak{H}^{\left(n_{\beta}\right)}\right) \mathrm{e}^{\frac{1}{2}\left\langle\mathfrak{H}^{\left(n_{\beta}\right)}, \boldsymbol{\partial}_{P} \mathfrak{H}^{\left(n_{\beta}\right)}\right\rangle+\frac{\beta}{2 n}\left\langle\mathfrak{H}^{(n)}, \hat{H} \mathfrak{H}^{(n)}\right\rangle} \mathrm{e}^{\frac{\beta s}{n} \sum_{k=n}^{n_{\beta}-1} \varkappa_{P}^{(k)}(K)-\frac{\beta}{n} \sum_{k=0}^{n-1} \varkappa_{P}^{(k)}(W)} .
\end{aligned}
$$

Therefore, by Definition 3.6, it suffices to combine (78) with (80) to get the assertion.

Corollary 4.8 refers to a Feynman-Kac-like formula by viewing the variable $k \in\{0, \ldots, n-1\}$ in the definition (63) of the finite-dimensional Hilbert space $\mathfrak{H}^{(n)}, n \in \mathbb{N}$, as a "time coordinate". Each element of a (copied) orthonormal basis $\left\{\psi_{i}^{(k)}\right\}_{i \in I}$ of $\mathcal{H}^{(k)}$ is associated with a "space coordinate" $i \in I$. In this context, the normal operator $\partial$ defined by (65) is a discrete-time derivative operator, which, by Equation (66), has the self-dual version $\boldsymbol{\partial}_{P}$.

\section{Properties of the Covariance}

\subsection{Pfaffian Bounds}

Having in mind further applications of the Feynman-Kac-like formula of Corollary 4.8, we aim to bound Pfaffians of the form

$$
\operatorname{Pf}\left[\mathfrak{M}_{j_{q}, j_{l}}\left\langle\mathfrak{A} \varphi_{q}^{\left(k_{q}\right)}, C_{H, P}^{(n)} \varphi_{l}^{\left(k_{l}\right)}\right\rangle_{\mathfrak{H}^{\left(n_{\beta}\right)}}\right]_{q, l=1}^{2 N}
$$


for $\beta \in \mathbb{R}^{+}, n, m, N \in \mathbb{N}$ with $n$ being sufficiently large, $\mathfrak{M} \in \operatorname{Mat}(m, \mathbb{R})$ with $\mathfrak{M} \geq 0$, and all

$$
\left\{\left(k_{q}, \varphi_{q}, j_{q}\right)\right\}_{q=1}^{2 N} \subset\left\{0, \ldots, n_{\beta}-1\right\} \times \mathcal{H} \times\{1, \ldots, m\} .
$$

In (81), $P$ is a basis projection diagonalizing a self-dual Hamiltonian $H$ on $(\mathcal{H}, \mathfrak{A})$. Recall that $n_{\beta} \doteq n+\lfloor n / \beta\rfloor$ and $C_{H, P}^{(n)}$ is the covariance defined by (76) for integers $n>\beta\|H\|_{\mathcal{B}(\mathcal{H})}$. Observe also that the matrix $\mathfrak{M}$ is symmetric, for it is positive and, hence, the $2 N \times 2 N$ matrix in (81) is skew-symmetric.

The positive, real matrix $\mathfrak{M}$ appears in the so-called Brydges-Kennedy tree expansions. See, e.g., [BdSP17b, Section 1.3] and [BdSPX] for more details. For previous applications of this expansion, see also [BGPS94, Section 3], [GM10, Section 3.2], and more recently [GMP16, Section 5.A.]. In all previous results, the covariance refers to the special case $k<n$ in Corollary 4.8. Moreover, as explained in Section 1, in our approach, multiscale analysis is not necessary to treat the Matsubara UV problem.

We start by bounding determinants of the form

$$
\operatorname{det}\left[\left\langle\varphi_{q}^{\left(k_{q}\right)}, C_{H, P}^{(n)} \varphi_{N+l}^{\left(k_{N+l}\right)}\right\rangle_{\mathfrak{H}^{\left(n_{\beta}\right)}}\right]_{q, l=1}^{N}
$$

for $\beta \in \mathbb{R}^{+}, n, N \in \mathbb{N}$ with $n$ being sufficiently large, and all

$$
\left\{\left(k_{q}, \varphi_{q}\right)\right\}_{q=1}^{2 N} \subset\left\{0, \ldots, n_{\beta}-1\right\} \times \mathfrak{h}_{P} .
$$

Then, we show below that the general case $m \in \mathbb{N}$ can always be reduced to the situation $m=1$ and $\mathfrak{M}=1$, by redefining the self-dual Hilbert space $\mathcal{H}$, while, for $m=1$ and $\mathfrak{M}=1$, the Pfaffian (81) can be written as the sum of $2^{2 N}$ determinants of the form (82). See Proposition 3.7.

The ingredients to bound (82) are: (I) Corollary 3.8, which states that

$$
\operatorname{det}\left[\left\langle\varphi_{q}^{\left(k_{q}\right)}, C_{H, P}^{(n)} \varphi_{N+l}^{\left(k_{N+l}\right)}\right\rangle_{\mathfrak{H}^{\left(n_{\beta}\right)}}\right]_{q, l=1}^{N} \mathbf{1}=\int \mathrm{d} \mu_{C_{H, P}^{(n)}}\left(\mathfrak{H}^{\left(n_{\beta}\right)}\right)\left(\varphi_{1}^{*}\right)^{\left(k_{1}\right)} \cdots\left(\varphi_{N}^{*}\right)^{\left(k_{N}\right)} \varphi_{2 N}^{\left(k_{2 N}\right)} \cdots \varphi_{N+1}^{\left(k_{N+1}\right)},
$$

where $\varphi^{*} \doteq \mathfrak{A} \varphi$ for all $\varphi \in \mathcal{H}$ (Definition 3.13); (II) the relationship between the exterior product $\wedge$ and the circle product $\circ_{P}$ as stated in Lemma 3.10 (iv)-(v); (III) the Chernoff product approximation of bilinear elements of the self-dual CAR algebra explained in Lemma 4.5; (IV) the tracial state formula (Theorem 4.1), which allows us to represent determinants (83) as traces; and (V) Hölder inequalities for Schatten norms. This approach based on Grassmann-algebra methods turns out to be more efficient, in the finite-dimensional case, than the construction done in [BdSP17b], which involves quasi-free states in suitably-chosen CAR algebras.

We define Schatten norms in the context of self-dual CAR algebra by

$$
\|A\|_{s} \doteq\left(\operatorname{tr}\left(|A|^{s}\right)\right)^{\frac{1}{s}}, \quad A \in \operatorname{sCAR}(\mathcal{H}, \mathfrak{A}), s \geq 1
$$

and

$$
\|A\|_{\infty} \doteq \lim _{s \rightarrow \infty}\left(\operatorname{tr}\left(|A|^{s}\right)\right)^{\frac{1}{s}}=\|A\|_{\operatorname{sCAR}(\mathcal{H}, \mathfrak{A})}, \quad A \in \operatorname{sCAR}(\mathcal{H}, \mathfrak{A})
$$

where $|A| \doteq\left(A^{*} A\right)^{1 / 2}$ and $\operatorname{tr} \in \operatorname{SCAR}(\mathcal{H}, \mathfrak{A})^{*}$ is the tracial state of Definition 2.9. See also Remark 6.4. Hölder inequalities for Schatten norms then refer to the following bounds: For any $m \in \mathbb{N}$, $r, s_{1}, \ldots, s_{m} \in[1, \infty]$ such that $\sum_{j=1}^{m} 1 / s_{j}=1 / r$, and all elements $A_{1}, \ldots, A_{m} \in \operatorname{sCAR}(\mathcal{H}, \mathfrak{A})$,

$$
\left\|A_{1} \cdots A_{m}\right\|_{r} \leq \prod_{j=1}^{m}\left\|A_{j}\right\|_{s_{j}} .
$$

This type of inequality, combined with (83) and Lemmata 3.10 and 4.5, yields a sharp bound on the determinant (82): 


\section{Theorem 5.1 (Determinant bounds)}

Let $H$ be a self-dual Hamiltonian on $(\mathcal{H}, \mathfrak{A})$ and take any basis projection $P$ diagonalizing $H$. Then, for $\beta \in \mathbb{R}^{+}, n, N \in \mathbb{N}$ with $n>\beta\|H\|_{\mathcal{B}(\mathcal{H})}$, all $k_{1}, \ldots, k_{2 N} \in\left\{0, \ldots, n_{\beta}-1\right\}\left(n_{\beta} \doteq n+\lfloor n / \beta\rfloor\right)$ and $\varphi_{1}, \ldots, \varphi_{2 N} \in \mathfrak{h}_{P}$, the following bound holds true:

$$
\left|\operatorname{det}\left[\left\langle\varphi_{q}^{\left(k_{q}\right)}, C_{H, P}^{(n)} \varphi_{N+l}^{\left(k_{N+l}\right)}\right\rangle_{\mathfrak{H}^{\left(n_{\beta}\right)}}\right]_{q, l=1}^{N}\right| \leq \prod_{q=1}^{2 N}\left\|\varphi_{q}\right\|_{\mathcal{H}} .
$$

Proof. Fix all parameters of the theorem. Pick any orthonormal basis $\left\{\psi_{j}\right\}_{j \in J} \subset \mathfrak{h}_{P}$ of eigenvectors of $H_{P} \doteq 2 P H P \in \mathcal{B}\left(\mathfrak{h}_{P}\right)$. Similar to the derivation of (77), by Lemmata 3.10 (iv) and 4.5, together with Condition (c) (Definition 2.1), Definition 3.16, and Equation (34), for any $n_{1}, n_{2} \in \mathbb{N}$, and all indices $j_{1}, \ldots, j_{n_{1}+n_{2}} \in J$,

$$
\begin{aligned}
& \psi_{j_{1}}^{*} \cdots \psi_{j_{n_{1}}}^{*} \varkappa_{P}\left(\left[X_{\beta\langle\mathrm{B}, H \mathrm{~B}\rangle / 2}^{(n)}\right]^{\frac{1}{n}}\right) \psi_{j_{n_{1}+1}} \cdots \psi_{j_{n_{1}+n_{2}}} \\
= & \varkappa_{P}\left(\mathrm{~B}\left(\psi_{j_{1}}\right) \cdots \mathrm{B}\left(\psi_{j_{n_{1}}}\right)\left[X_{\beta\langle\mathrm{B}, H \mathrm{~B}\rangle / 2}^{(n)}\right]^{\frac{1}{n}} \mathrm{~B}\left(\psi_{j_{n_{1}+1}}\right)^{*} \cdots \mathrm{B}\left(\psi_{j_{n_{1}+n_{2}}}\right)^{*}\right) .
\end{aligned}
$$

By taking linear combinations of the above equalities, we thus deduce that, for any $n_{1}, n_{2} \in \mathbb{N}$ and all $\varphi_{1}, \ldots, \varphi_{n_{1}+n_{2}} \in \mathfrak{h}_{P}$

$$
\begin{aligned}
& \varphi_{1}^{*} \cdots \varphi_{n_{1}}^{*} \varkappa_{P}\left(\left[X_{\beta\langle\mathrm{B}, H \mathrm{~B}\rangle / 2}^{(n)}\right]^{\frac{1}{n}}\right) \varphi_{n_{1}+1} \cdots \varphi_{n_{1}+n_{2}} \\
= & \varkappa_{P}\left(\mathrm{~B}\left(\varphi_{1}\right) \cdots \mathrm{B}\left(\varphi_{n_{1}}\right)\left[X_{\beta\langle\mathrm{B}, H \mathrm{~B}\rangle / 2}^{(n)}\right]^{\frac{1}{n}} \mathrm{~B}\left(\varphi_{n_{1}+1}\right)^{*} \cdots \mathrm{B}\left(\varphi_{n_{1}+n_{2}}\right)^{*}\right) .
\end{aligned}
$$

We are now in a position to write the determinant as a trace in order to next use Hölder inequalities: By Definition 3.6 and Equations (76)-(79) and (83),

$$
\operatorname{det}\left[\left\langle\varphi_{q}^{\left(k_{q}\right)}, C_{H, P}^{(n)} \varphi_{N+l}^{\left(k_{N+l}\right)}\right\rangle_{\mathfrak{H}^{\left(n_{\beta}\right)}}\right]_{q, l=1}^{N} \mathbf{1}=\frac{2^{-\frac{\operatorname{dim} \mathcal{H}}{2}}}{\operatorname{tr}\left(X_{\beta\langle\mathrm{B}, H \mathrm{~B}\rangle / 2}^{(n)}\right)} \int_{P} \mathrm{~d}\left(\mathfrak{H}^{\left(n_{\beta}\right)}\right) \mathrm{e}^{\frac{1}{2}\left\langle\mathfrak{H}^{\left(n_{\beta}\right)}, \boldsymbol{\partial}_{P} \mathfrak{H}^{\left(n_{\beta}\right)}\right\rangle} \mathfrak{Q}
$$

with

$$
\mathfrak{Q} \doteq\left(\prod_{k=0}^{n-1} \varkappa_{P}^{(k)}\left(\left[X_{\beta\langle\mathrm{B}, H \mathrm{~B}\rangle / 2}^{(n)}\right]^{\frac{1}{n}}\right)\right)\left(\varphi_{1}^{*}\right)^{\left(k_{1}\right)} \cdots\left(\varphi_{N}^{*}\right)^{\left(k_{N}\right)} \varphi_{2 N}^{\left(k_{2 N}\right)} \cdots \varphi_{N+1}^{\left(k_{N+1}\right)} .
$$

To get the assertion, it suffices now to reorganize the exterior products $\wedge$ in $\mathfrak{Q}$ by regrouping all terms associated with the same index $k \in\left\{0, \ldots, n_{\beta}-1\right\}$. Note that an additional minus sign can appear, because of the antisymmetry of $\wedge$. Doing this, we rewrite the element $\mathfrak{Q}$ in the form $\pm \varkappa_{P}^{(0)}\left(A_{0}\right) \wedge \cdots \wedge \varkappa_{P}^{\left(n_{\beta}-1\right)}\left(A_{n_{\beta}-1}\right)$ and, by Lemma 3.10 (iv) and Theorem 4.1, together with (88),

$$
\operatorname{det}\left[\left\langle\varphi_{q}^{\left(k_{q}\right)}, C_{H, P}^{(n)} \varphi_{N+l}^{\left(k_{N+l}\right)}\right\rangle_{\mathfrak{H}^{\left(n_{\beta}\right)}}\right]_{q, l=1}^{N}= \pm \frac{1}{\operatorname{tr}\left(X_{\beta\langle\mathrm{B}, H \mathrm{~B}\rangle / 2}^{(n)}\right)} \operatorname{tr}\left(A_{0} \cdots A_{n_{\beta}-1}\right) .
$$

More precisely, using Definition 3.16 and (62), for any $k \notin\left\{k_{1}, \ldots, k_{2 N}\right\}$,

$$
A_{k}=\left[X_{\beta\langle\mathrm{B}, H \mathrm{~B}\rangle / 2}^{(n)}\right]^{\frac{1}{n}}
$$

unless $k \in\left\{n, \ldots, n_{\beta}-1\right\}$ in which case $A_{k}=1$. If $k \in\left\{k_{1}, \ldots, k_{2 N}\right\}$ then $\varkappa_{P}\left(A_{k}\right)$ is of the form (87), with $H$ being zero whenever $k \in\left\{n, \ldots, n_{\beta}-1\right\}$. Therefore, one can apply the inequality

$$
|\operatorname{tr}(B)| \leq \operatorname{tr}(|B|), \quad B \in \operatorname{sCAR}(\mathcal{H}, \mathfrak{A})
$$


and Hölder inequalities (86) to the right-hand side of (89), with $m=n+2 N, r=1, s_{j}=n$ for each term $\left[X_{\beta\langle\mathrm{B}, H \mathrm{~B}\rangle / 2}^{(n)}\right]^{\frac{1}{n}}$ and $s_{j}=\infty$ for every generator $\mathrm{B}(\varphi)$ (where $\varphi \in \mathfrak{h}_{P}$ ). Since, by Remark 2.2, Lemma 4.5 and Equation (85),

$$
\left\|\left[X_{\beta\langle\mathrm{B}, H \mathrm{~B}\rangle / 2}^{(n)}\right]^{\frac{1}{n}}\right\|_{n}=\left(\operatorname{tr}\left(X_{\beta\langle\mathrm{B}, H \mathrm{~B}\rangle / 2}^{(n)}\right)\right)^{\frac{1}{n}} \quad \text { and } \quad\|\mathrm{B}(\varphi)\|_{\infty} \leq\|\varphi\|_{\mathcal{H}}
$$

the assertion then follows.

\section{Corollary 5.2 (Pfaffian bounds - I)}

Let $H$ be a self-dual Hamiltonian on $(\mathcal{H}, \mathfrak{A})$ and take any basis projection $P$ diagonalizing $H$. Then, for $\beta \in \mathbb{R}^{+}, n, N \in \mathbb{N}$ with $n>\beta\|H\|_{\mathcal{B}(\mathcal{H})}$, all $k_{1}, \ldots, k_{2 N} \in\left\{0, \ldots, n_{\beta}-1\right\}$ and $\varphi_{1}, \ldots, \varphi_{2 N} \in \mathcal{H}$, the following bound holds true:

$$
\left|\operatorname{Pf}\left[\left\langle\mathfrak{A} \varphi_{q}^{\left(k_{q}\right)}, C_{H, P}^{(n)} \varphi_{l}^{\left(k_{l}\right)}\right\rangle_{\mathfrak{H}^{\left(n_{\beta}\right)}}\right]_{q, l=1}^{2 N}\right| \leq \prod_{q=1}^{2 N}\left(\left\|P \varphi_{q}\right\|_{\mathcal{H}}+\left\|P^{\perp} \varphi_{q}\right\|_{\mathcal{H}}\right) .
$$

Proof. The proof is a direct consequence of Proposition 3.7, Theorem 5.1, Equations (12), (13) and (83), together with the fact that $\hat{P}$ diagonalizes the self-dual operator $C_{H, P}^{(n)}$.

Bounds for general Pfaffians of the form (81) can be deduced from Corollary 5.2, which corresponds to the special case $m=1$ and $\mathfrak{M}=1$. This is done by extending the self-dual Hilbert space $(\mathcal{H}, \mathfrak{A})$, similar to what is done in [BdSP17b, Section 1.3]: For any fixed $m \in \mathbb{N}$, a (generic) nonvanishing real positive matrix $\mathfrak{M} \in \operatorname{Mat}(m, \mathbb{R})$ gives rise to a positive sesquilinear form on $\mathbb{C}^{m}$ defined by

$$
\left\langle\left(x_{1}, \ldots, x_{m}\right),\left(y_{1}, \ldots, y_{m}\right)\right\rangle_{\mathbb{C}^{m}}^{\mathfrak{M}} \doteq \sum_{j, l=1}^{m} \overline{x_{j}} y_{l} \mathfrak{M}_{j, l} .
$$

In general, this sesquilinear form is degenerated. Then, one can define a Hilbert space

$$
\mathbb{M} \doteq \mathbb{C}^{m} /\left\{x \in \mathbb{C}^{m}:\langle x, x\rangle_{\mathbb{C}^{m}}^{\mathfrak{M}}=0\right\}
$$

whose scalar product is defined by

$$
\langle[x],[y]\rangle_{\mathbb{M}} \doteq\langle x, y\rangle_{\mathbb{C}^{m}}^{\mathfrak{M}}, \quad x, y \in \mathbb{C}^{m} .
$$

The map from $\mathbb{C}^{m}$ to itself defined by

$$
x=\left(x_{1}, \ldots, x_{m}\right) \mapsto \bar{x} \doteq\left(\overline{x_{1}}, \ldots, \overline{x_{m}}\right)
$$

yields an antiunitary involution $[x] \mapsto[\bar{x}]$ on $\mathbb{M}$ denoted by $\mathbb{A}$.

Then, it suffices to replace $(\mathcal{H}, \mathfrak{A})$ with $(\mathcal{H} \otimes \mathbb{M}, \mathfrak{A} \otimes \mathbb{A})$ to get, from Corollary 5.2, bounds for Pfaffians of the form (81):

\section{Corollary 5.3 (Pfaffian bounds - II)}

Let $H$ be a self-dual Hamiltonian on $(\mathcal{H}, \mathfrak{A})$ and take any basis projection $P$ diagonalizing $H$. Then, for $\beta \in \mathbb{R}^{+}, n, m, N \in \mathbb{N}$ with $n>\beta\|H\|_{\mathcal{B}(\mathcal{H})}, \mathfrak{M} \in \operatorname{Mat}(m, \mathbb{R})$ with $\mathfrak{M} \geq 0$, and all

$$
\left\{\left(k_{q}, \varphi_{q}, j_{q}\right)\right\}_{q=1}^{2 N} \subset\left\{0, \ldots, n_{\beta}-1\right\} \times \mathcal{H} \times\{1, \ldots, m\},
$$

the following bound holds true:

$$
\begin{aligned}
\left|\operatorname{Pf}\left[\mathfrak{M}_{j_{q}, j_{l}}\left\langle\mathfrak{A} \varphi_{q}^{\left(k_{q}\right)}, C_{H, P}^{(n)} \varphi_{l}^{\left(k_{l}\right)}\right\rangle_{\mathfrak{H}^{\left(n_{\beta}\right)}}\right]_{q, l=1}^{2 N}\right| & \leq \prod_{q=1}^{2 N}\left(\left\|P \varphi_{q}\right\|_{\mathcal{H}}+\left\|P^{\perp} \varphi_{q}\right\|_{\mathcal{H}}\right) \mathfrak{M}_{j_{q}, j_{q}}^{1 / 2} \\
& \leq 2^{N} \prod_{q=1}^{2 N}\left\|\varphi_{q}\right\|_{\mathcal{H}} \mathfrak{M}_{j_{q}, j_{q}}^{1 / 2} .
\end{aligned}
$$


Proof. Fix $m \in \mathbb{N}$ and $\mathfrak{M} \in \operatorname{Mat}(m, \mathbb{R})$ with $\mathfrak{M} \geq 0$. Let $\tilde{\mathcal{H}} \doteq \mathcal{H} \otimes \mathbb{M}$ and $\tilde{\mathfrak{A}} \doteq \mathfrak{A} \otimes \mathbb{A}$. Any operator $A \in \mathcal{B}(\mathcal{H})$ can be extended to an operator acting on $\tilde{\mathcal{H}}$ by the definition $\tilde{A} \doteq A \otimes \mathbf{1}_{\mathbb{M}} \in \mathcal{B}(\tilde{\mathcal{H}})$. A basis projection $P\left(\right.$ range $\left.\mathfrak{h}_{P}\right)$ associated with $(\mathcal{H}, \mathfrak{A})$ yields a basis projection $\tilde{P}$ associated with $(\tilde{\mathcal{H}}, \tilde{\mathfrak{A}})$, whose range is $\mathfrak{h}_{\tilde{P}}=\mathfrak{h}_{P} \otimes \mathbb{M}$. See Definition 2.4. If $H$ is a self-dual Hamiltonian on $(\mathcal{H}, \mathfrak{A})$ (Definition 2.7), then $\tilde{H}$ is also a self-dual Hamiltonian on $(\tilde{\mathcal{H}}, \tilde{\mathfrak{A}})$, with the same norm. If the basis projection $P$ diagonalizes $H$ then $\tilde{P}$ also diagonalizes $\tilde{H}$.

Meanwhile, we use the canonical identification

$$
\tilde{\mathfrak{H}}^{(n)} \doteq \bigoplus_{k=0}^{n-1} \tilde{\mathcal{H}}^{(k)} \equiv \mathfrak{H}^{(n)} \otimes \mathbb{M}, \quad n \in \mathbb{N},
$$

via the unitary map uniquely defined by the conditions

$$
(\varphi \otimes[x])^{(k)} \mapsto \varphi^{(k)} \otimes[x], \quad \varphi \in \mathcal{H}, x \in \mathbb{C}^{m},
$$

for any $k \in\{0, \ldots, n-1\}$. It is then straightforward to verify that

$$
C_{\tilde{H}, \tilde{P}}^{(n)}=C_{H, P}^{(n)} \otimes \mathbf{1}_{\mathbb{M}}
$$

See Equations (63), (66) and (76).

Now, using the notation $\mathfrak{e}_{j} \doteq\left[e_{j}\right] \in \mathbb{M}$, where $\left\{e_{j}\right\}_{j=1}^{m}$ is the canonical basis of $\mathbb{C}^{m}$, note that

$$
\mathfrak{M}_{j, l}=\left\langle\mathfrak{e}_{j}, \mathfrak{e}_{l}\right\rangle_{\mathbb{M}}, \quad j, l \in\{1, \ldots, m\} .
$$

Therefore, if $H$ is a self-dual Hamiltonian on $(\mathcal{H}, \mathfrak{A})$ and $P$ any basis projection $P$ diagonalizing $H$,

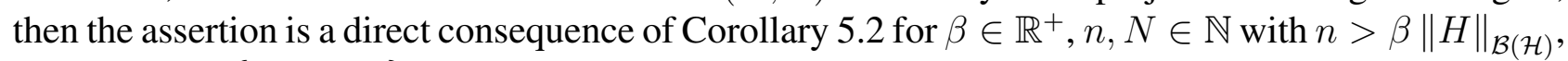
$j_{1}, \ldots, j_{2 N} \in\{1, \ldots, m\}$, and all elements

$$
\left\{\left(k_{q}, \varphi_{q} \otimes \mathfrak{e}_{j_{q}}\right)\right\}_{q=1}^{2 N} \subset\left\{0, \ldots, n_{\beta}-1\right\} \times \mathfrak{h}_{\tilde{P}}
$$

Remark from (91) that

$$
\left\|\tilde{P}\left(\varphi \otimes \mathfrak{e}_{j}\right)\right\|_{\tilde{\mathcal{H}}}+\left\|\tilde{P}^{\perp}\left(\varphi \otimes \mathfrak{e}_{j}\right)\right\|_{\tilde{\mathcal{H}}}=\left(\|P \varphi\|_{\mathcal{H}}+\left\|P^{\perp} \varphi\right\|_{\mathcal{H}}\right) \mathfrak{M}_{j, j}^{1 / 2}, \quad \varphi \in \mathcal{H}, j \in\{1, \ldots, m\}
$$

\section{Remark 5.4}

Similar to [BdSP17b, Corollary 1.6] one can check that the bound on Pfaffians obtained in Corollary 5.3 is sharp if $\varphi_{1}, \ldots, \varphi_{2 N} \in \mathfrak{h}_{P} \cup \mathfrak{h}_{P^{\perp}}$, in the sense given in [BdSP17b, Equation (14)]. In particular, the general Pfaffian bound of Corollary 5.3 is sharp up to a factor of $2^{N}$.

\subsection{Covariances as Correlations of Quasi-Free States}

Fix, in all this subsection, an inverse temperature $\beta \in \mathbb{R}_{0}^{+}$, some self-dual Hamiltonian $H$ on $(\mathcal{H}, \mathfrak{A})$, a basis projection $P$ diagonalizing $H$, and $n \in \mathbb{N}$ so that $n>\beta\|H\|_{\mathcal{B}(\mathcal{H})}$. Using the trace representation (89) of determinants associated with covariances, one can rewrite the matrix elements

$$
\left\langle\hat{\varphi}_{1}, C_{H, P}^{(n)} \hat{\varphi}_{2}\right\rangle_{\mathfrak{H}^{\left(n_{\beta}\right)}}, \quad \hat{\varphi}_{1}, \hat{\varphi}_{2} \in \mathfrak{H}^{\left(n_{\beta}\right)}
$$

in terms of space-time correlations of a quasi-free state.

Using the map $\mathbf{K}: \mathcal{B}(\mathcal{H}) \rightarrow \mathcal{B}(\mathcal{H})(21)$, we define a sequence of self-dual Hamiltonians on $(\mathcal{H}, \mathfrak{A})$ by

$$
H^{(n)} \doteq \beta^{-1} n \mathbf{K}\left(\ln \left(1+n^{-1} \beta H\right)\right)=\frac{\beta^{-1} n}{2} \ln \left(\frac{1+n^{-1} \beta H}{1-n^{-1} \beta H}\right)
$$


for any $n>\beta\|H\|_{\mathcal{B}(\mathcal{H})}$. If the basis projection $P$ diagonalizes $H$ then it also diagonalizes $H^{(n)}$. In view of the trace representation (89) and Lemma 2.10, this Hamitonian is constructed such that the equality

$$
X_{\beta\langle\mathrm{B}, H \mathrm{~B}\rangle / 2}^{(n)}=D \mathrm{e}^{\beta\left\langle\mathrm{B}, H^{(n)} \mathrm{B}\right\rangle / 2}, \quad \beta \in \mathbb{R}_{0}^{+},
$$

holds true for some finite constant $D>0$. See (46) and Lemma 4.5. Observe meanwhile that $H^{(n)}$ is an approximation of $H$ : There is a finite constant $D>0$ such that, for $\beta \in \mathbb{R}_{0}^{+}$and $n>2 \beta\|H\|_{\mathcal{B}(\mathcal{H})}$,

$$
\left\|H^{(n)}-H\right\|_{\mathcal{B}(\mathcal{H})} \leq D n^{-2} \beta^{2}\|H\|_{\mathcal{B}(\mathcal{H})}^{3} .
$$

Compare also (93) with [BdSP17b, Eqs. (16)-(17)].

Then, using the definition $[x]_{+} \doteq(x+|x|) / 2$ for $x \in \mathbb{R}$ and

$$
\alpha_{n}(k, q) \doteq \beta n^{-1}[\min \{n-k, q-k\}]_{+} \in[0, \beta], \quad k, q \in \mathbb{Z},
$$

we rewrite the matrix elements (92), at fixed time coordinates, in terms of space-time correlations of the quasi-free states $\rho_{H^{(n)}}$ with symbol $\left(1+\mathrm{e}^{-\beta H^{(n)}}\right)^{-1}$ (see (26)-(27) and (29)-(30)):

\section{Theorem 5.5 (Covariances as correlations of quasi-free states)}

Let $H$ be a self-dual Hamiltonian on $(\mathcal{H}, \mathfrak{A})$ and take any basis projection $P$ diagonalizing $H$. Then, for any $\beta \in \mathbb{R}^{+}, n \in \mathbb{N}$ with $n>\beta\|H\|_{\mathcal{B}(\mathcal{H})}, k_{1}, k_{2} \in\left\{0, \ldots, n_{\beta}-1\right\}$ and $\varphi_{1}, \varphi_{2} \in \mathcal{H}$,

$$
\left\langle\varphi_{1}^{\left(k_{1}\right)}, C_{H, P}^{(n)} \varphi_{2}^{\left(k_{2}\right)}\right\rangle_{\mathfrak{H}^{\left(n_{\beta}\right)}}=\left\langle\varphi_{1}, \mathfrak{C}_{H, P}^{(n)}\left(k_{1}, k_{2}\right) \varphi_{2}\right\rangle_{\mathcal{H}},
$$

where

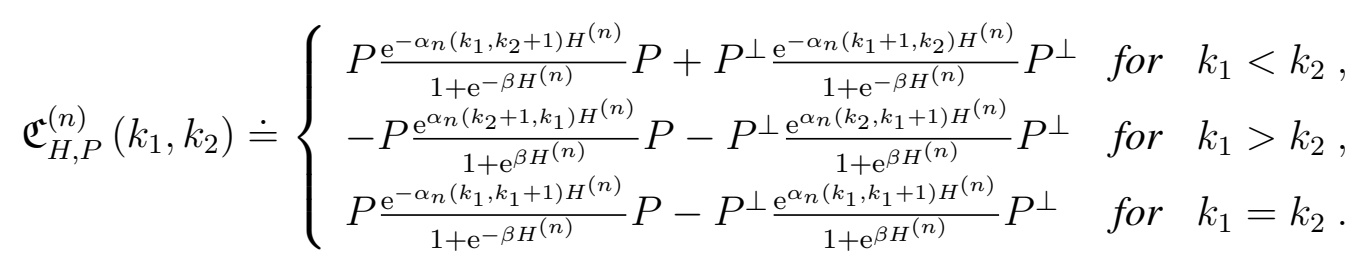

Proof. Fix all parameters of the theorem. By construction, the covariance $C_{H, P}^{(n)}$ is a self-dual operator on $\left(\mathfrak{H}^{\left(n_{\beta}\right)}, \hat{\mathfrak{A}}\right)$, which is diagonalized by the extension $\hat{P}$ of $P$ (see (64) with $n_{\beta}$ replacing $n$ ). In particular, for any $\varphi_{1}, \varphi_{2} \in \mathcal{H}$ and $k_{1}, k_{2} \in\left\{0, \ldots, n_{\beta}-1\right\}$,

$$
\begin{aligned}
\left\langle\varphi_{1}^{\left(k_{1}\right)}, C_{H, P}^{(n)} \varphi_{2}^{\left(k_{2}\right)}\right\rangle_{\mathfrak{H}^{\left(n_{\beta}\right)}}= & \left\langle\left(P \varphi_{1}\right)^{\left(k_{1}\right)}, C_{H, P}^{(n)}\left(P \varphi_{2}\right)^{\left(k_{2}\right)}\right\rangle_{\mathfrak{H}^{\left(n_{\beta}\right)}} \\
& -\left\langle\left(P \mathfrak{A} \varphi_{2}\right)^{\left(k_{2}\right)}, C_{H, P}^{(n)}\left(P \mathfrak{A} \varphi_{1}\right)^{\left(k_{1}\right)}\right\rangle_{\mathfrak{H}^{\left(n_{\beta}\right)}} .
\end{aligned}
$$

Therefore, it suffices to do computations for $\varphi_{1}, \varphi_{2} \in \mathfrak{h}_{P}$ only. In this special case, it is straightforward, albeit cumbersome, to derive the following equalities from Equations (89) and (94), together with Lemmata 2.8 and 2.10: If $k_{1} \leq k_{2}$ then

$$
\left\langle\varphi_{1}^{\left(k_{1}\right)}, C_{H, P}^{(n)} \varphi_{2}^{\left(k_{2}\right)}\right\rangle_{\mathfrak{H}^{\left(n_{\beta}\right)}}=\rho_{H^{(n)}}\left(\mathrm{B}\left(\mathrm{e}^{-\alpha_{n}\left(k_{1}, k_{2}+1\right) H^{(n)}} \varphi_{1}\right) \mathrm{B}\left(\mathfrak{A} \varphi_{2}\right)\right)
$$

whereas if $k_{2}<k_{1}$,

$$
\left\langle\varphi_{1}^{\left(k_{1}\right)}, C_{H, P}^{(n)} \varphi_{2}^{\left(k_{2}\right)}\right\rangle_{\left.\mathfrak{H}^{(n \beta}\right)}=-\rho_{H^{(n)}}\left(\mathrm{B}\left(\mathfrak{A} \varphi_{2}\right) \mathrm{B}\left(\mathrm{e}^{\alpha_{n}\left(k_{2}+1, k_{1}\right) H} \varphi_{1}\right)\right) .
$$

By combining these two equalities for $\varphi_{1}, \varphi_{2} \in \mathfrak{h}_{P}$ with Equations (30) and (98), we directly get the theorem. Note additionally that, $C_{H, P}^{(n)}$ being self-dual, for all $k_{1}, k_{2} \in\left\{0, \ldots, n_{\beta}-1\right\}$,

$$
\mathfrak{C}_{H, P}^{(n)}\left(k_{2}, k_{1}\right)=-\mathfrak{A} \mathfrak{C}_{H, P}^{(n)}\left(k_{1}, k_{2}\right)^{*} \mathfrak{A} .
$$

This relation can be used to obtain the first line in the right-hand side of (97) from the second one and vice versa, or to check mutual consistency of these two lines. 


\subsection{Summability}

In this section we derive bounds on the decay of the covariances (76) for general fermion systems on the lattice, from the celebrated Combes-Thomas estimates. See also [dRS17] which has simultaneously been done and uses Combes-Thomas estimates.

\subsubsection{Lattice Fermion Systems in the Self-dual Formalism}

We consider fermion systems on the lattice $\mathbb{Z}^{d}(d \in \mathbb{N})$ with arbitrary (finite) spin set $\mathrm{S}$. We define the Hilbert spaces $\mathcal{H}_{\mathrm{S}} \doteq \ell^{2}(\mathrm{~S}) \oplus \ell^{2}(\mathrm{~S})^{*}$ and $\mathcal{H}_{L} \doteq \ell^{2}\left(\Lambda_{L} ; \mathcal{H}_{\mathrm{S}}\right)$ for all $L \in \mathbb{R}_{0}^{+} \cup\{\infty\}$, where

$$
\Lambda_{L} \doteq\left\{\left(x_{1}, \ldots, x_{d}\right) \in \mathbb{Z}^{d}:\left|x_{1}\right|, \ldots,\left|x_{d}\right| \leq L\right\}
$$

is a cubic box of side length $\mathcal{O}(L)$. See also Sections 6.2 and 8.1, in particular Equation (137).

Fix any antiunitary involution $\mathfrak{A}_{\mathrm{S}}$ on $\mathcal{H}_{\mathrm{S}}$. A canonical example is given by (116) for $\mathcal{H}=\mathcal{H}_{\mathrm{S}}$. For any $L \in \mathbb{R}_{0}^{+} \cup\{\infty\}$, we define an antiunitary involution $\mathfrak{A}_{L}$ on $\mathcal{H}_{L}$ by

$$
\left(\mathfrak{A}_{L} \varphi\right)(x) \doteq \mathfrak{A}_{\mathrm{S}}(\varphi(x)), \quad x \in \Lambda_{L}, \varphi \in \mathcal{H}_{L} .
$$

Then, $\left(\mathcal{H}_{L}, \mathfrak{A}_{L}\right)$ is a self-dual Hilbert space for any $L \in \mathbb{R}_{0}^{+} \cup\{\infty\}$. Note that $\mathcal{H}_{\mathrm{S}}$ and $\mathcal{H}_{L}$ are finite-dimensional, with even dimension, whenever $L<\infty$ : Let

$$
\mathbb{X}_{L} \doteq \Lambda_{L} \times \mathrm{S} \times\{+,-\}, \quad L \in \mathbb{R}_{0}^{+} \cup\{\infty\}
$$

The canonical orthonormal basis $\left\{\mathfrak{e}_{\mathbf{x}}\right\}_{\mathbf{x} \in \mathbb{X}_{L}}$ of $\mathcal{H}_{L}, L \in \mathbb{R}_{0}^{+} \cup\{\infty\}$, is defined by

$$
\mathfrak{e}_{\mathbf{x}}(y) \doteq \delta_{x, y} \mathfrak{f}_{\mathrm{s}, v}, \quad \mathbf{x}=(x, \mathrm{~s}, v) \in \mathbb{X}_{L}, \quad y \in \Lambda_{L},
$$

where $\mathfrak{f}_{\mathrm{s},+} \doteq \mathfrak{A}_{\mathrm{S}} \mathfrak{f}_{\mathrm{s},-} \in \mathcal{H}_{\mathrm{S}}$ and $\mathfrak{f}_{\mathrm{s},-}(\mathrm{t}) \doteq \delta_{\mathrm{s}, \mathrm{t}}$ for any $\mathrm{s}, \mathrm{t} \in \mathrm{S}$.

In the self-dual formalism, a lattice fermion system in infinite volume is defined by a self-dual Hamiltonian $H_{\infty} \in \mathcal{B}\left(\mathcal{H}_{\infty}\right)$ on $\left(\mathcal{H}_{\infty}, \mathfrak{A}_{\infty}\right)$, that is, $H_{\infty}=H_{\infty}^{*}=-\mathfrak{A}_{\infty} H_{\infty} \mathfrak{A}_{\infty}$. See Definition 2.7 which is here extended to the infinite-dimensional case. For a fixed basis projection $P_{\infty}$ diagonalizing $H_{\infty}$, the operator $P_{\infty} H_{\infty} P_{\infty}$ is the so-called one-particle Hamiltonian associated with the system, as already explained at the end of Section 2.2. See also Section 6.3. To obtain the corresponding self-dual Hamiltonians in finite volume we use the orthogonal projector $P_{\mathcal{H}_{L}} \in \mathcal{B}\left(\mathcal{H}_{\infty}\right)$ on $\mathcal{H}_{L}$ and define

$$
H_{L} \doteq P_{\mathcal{H}_{L}} H_{\infty} P_{\mathcal{H}_{L}}, \quad L \in \mathbb{R}_{0}^{+} .
$$

By construction, if $H_{\infty}$ is a self-dual Hamiltonian on $\left(\mathcal{H}_{\infty}, \mathfrak{A}_{\infty}\right)$, then, for any $L \in \mathbb{R}_{0}^{+}, H_{L}$ is a self-dual Hamiltonian on $\left(\mathcal{H}_{L}, \mathfrak{A}_{L}\right)$.

\subsubsection{Combes-Thomas Estimate and Summability of the Fermi Distribution}

We present a version of the Combes-Thomas estimate, first proven in [CT73], that is adapted to the present setting: Fix $\epsilon \in(0,1]$. Given a self-adjoint $H=H^{*} \in \mathcal{B}\left(\mathcal{H}_{\infty}\right)$ whose spectrum is denoted by $\operatorname{spec}(H)$, we define the constants

$$
\mathbf{S}(H, \mu) \doteq \sup _{\mathbf{x}_{1}=\left(x_{1}, \mathrm{~s}_{1}, v_{1}\right) \in \mathbb{X}_{\infty}} \sum_{\mathbf{x}_{2}=\left(x_{2}, \mathrm{~s}_{2}, v_{2}\right) \in \mathbb{X}_{\infty}}\left(\mathrm{e}^{\mu\left|x_{1}-x_{2}\right|^{\epsilon}}-1\right)\left|\left\langle\mathfrak{e}_{\mathbf{x}_{1}}, H \mathfrak{e}_{\mathbf{x}_{2}}\right\rangle\right| \in \mathbb{R}_{0}^{+} \cup\{\infty\},
$$

for $\mu \in \mathbb{R}_{0}^{+}$, and

$$
\Delta(H, z) \doteq \inf \{|z-\lambda|: \lambda \in \operatorname{spec}(H)\}, \quad z \in \mathbb{C},
$$


as the distance from the point $z$ to the spectrum of $H$. Since the function $x \mapsto\left(e^{x r}-1\right) / x$ is increasing on $\mathbb{R}^{+}$for any fixed $r \geq 0$, it follows that

$$
\mathbf{S}\left(H, \mu_{1}\right) \leq \frac{\mu_{1}}{\mu_{2}} \mathbf{S}\left(H, \mu_{2}\right), \quad \mu_{2} \geq \mu_{1} \geq 0
$$

The Combes-Thomas estimate we use is the following:

\section{Theorem 5.6 (Combes-Thomas)}

Let $\epsilon \in(0,1], H=H^{*} \in \mathcal{B}\left(\mathcal{H}_{\infty}\right), \mu \in \mathbb{R}_{0}^{+}$and $z \in \mathbb{C}$. If $\Delta(H, z)>\mathbf{S}(H, \mu)$ then

$$
\left|\left\langle\mathfrak{e}_{\mathbf{x}},(z-H)^{-1} \mathfrak{e}_{\mathbf{y}}\right\rangle\right| \leq \frac{\mathrm{e}^{-\mu|x-y|^{\epsilon}}}{\Delta(H, z)-\mathbf{S}(H, \mu)}, \quad \mathbf{x}=(x, \mathrm{~s}, v), \mathbf{y}=(y, \mathrm{t}, w) \in \mathbb{X}_{\infty}
$$

Proof. This proposition is a version of the first part of [AW16, Theorem 10.5] and is proven in the same way, because, for $\epsilon \in(0,1], \mathbf{x}=(x, \mathrm{~s}, v)$ and $\mathbf{y}=(y, \mathrm{t}, w), d_{\epsilon}(\mathbf{x}, \mathbf{y}) \doteq|x-y|^{\epsilon}$ defines a pseudometric on $\mathbb{X}_{\infty}$.

\section{Corollary 5.7 (Bound on differences of resolvents)}

Let $\epsilon \in(0,1], H=H^{*} \in \mathcal{B}\left(\mathcal{H}_{\infty}\right), \mu \in \mathbb{R}_{0}^{+}$and $\eta \in \mathbb{R}^{+}$such that $\mathbf{S}(H, \mu) \leq \eta / 2$. Then, for all $\mathbf{x}=(x, \mathrm{~s}, v), \mathbf{y}=(y, \mathrm{t}, w) \in \mathbb{X}_{\infty}$ and $u \in \mathbb{R}$,

$$
\begin{aligned}
& \left|\left\langle\mathfrak{e}_{\mathbf{x}},\left((H-u)^{2}+\eta^{2}\right)^{-1} \mathfrak{e}_{\mathbf{y}}\right\rangle\right| \\
\leq & 12 \mathrm{e}^{-\mu|x-y|^{\epsilon}}\left\langle\mathfrak{e}_{\mathbf{x}},\left((H-u)^{2}+\eta^{2}\right)^{-1} \mathfrak{e}_{\mathbf{x}}\right\rangle^{1 / 2}\left\langle\mathfrak{e}_{\mathbf{y}},\left((H-u)^{2}+\eta^{2}\right)^{-1} \mathfrak{e}_{\mathbf{y}}\right\rangle^{1 / 2} .
\end{aligned}
$$

Proof. This is proven like [AG98, Lemma 3], by replacing the euclidean metric of $\mathbb{Z}^{d}$ with the pseudometric of $\mathbb{X}_{\infty}$, as above. See also [AW16, Chapter 13].

We are now in a position to prove the summability of the Fermi distribution:

\section{Lemma 5.8 (Summability of the Fermi distribution)}

Fix a (finite) spin set $\mathrm{S}, d \in \mathbb{N}, \beta \in \mathbb{R}^{+}, \epsilon \in(0,1], v \in \mathbb{R}_{0}^{+}$and $H=H^{*} \in \mathcal{B}\left(\mathcal{H}_{\infty}\right)$. Then, for all $\mathbf{x}=(x, \mathrm{~s}, v)$ and $\mathbf{y}=(y, \mathrm{t}, w) \in \mathbb{X}_{\infty}$,

$$
\begin{array}{r}
\mathbf{D}_{H, \beta, v, \epsilon} \doteq \sup _{\alpha \in[0, \beta]} \sup _{\mathbf{x} \in \mathbb{X}_{\infty}}\left\{\sum_{\mathbf{y} \in \mathbb{X}_{\infty}} \mathrm{e}^{v|x-y|^{\epsilon}}\left|\left\langle\mathfrak{e}_{\mathbf{x}}, \frac{\mathrm{e}^{\alpha H}}{1+\mathrm{e}^{\beta H}} \mathfrak{e}_{\mathbf{y}}\right\rangle_{\mathcal{H}_{\infty}}\right|\right\} \\
\leq 96|\mathrm{~S}| \inf _{\mu \in \mathbb{R}_{0}^{+}} \inf _{\epsilon \in(0,1]} \sum_{x \in \mathbb{Z}^{d}} \mathrm{e}^{\left(v-\mu \min \left\{1, \frac{\pi}{4 \beta \mathbf{S}(H, \mu)}\right\}\right)|x|^{\epsilon}} .
\end{array}
$$

In particular, for $\mu=2 v$ and $v=\beta^{-1} \mu_{0} \in \mathbb{R}^{+}$, where $\mu_{0} \in \mathbb{R}^{+}$is such that $8 \mathbf{S}\left(H, \mu_{0}\right) \leq \pi$, we have $\mathbf{D}_{H, \beta, v, \epsilon}=\mathcal{O}\left((\beta+1)^{d \epsilon^{-1}}\right)$.

Proof. The proof is a simple adaptation of the one from [AG98, Theorem 3]: Fix all parameters of the lemma and observe that Corollary 5.7 combined with Inequality (103) yields

$$
\begin{aligned}
& \left|\left\langle\mathfrak{e}_{\mathbf{x}},\left((H-u)^{2}+\eta^{2}\right)^{-1} \mathfrak{e}_{\mathbf{y}}\right\rangle\right| \\
\leq & 12 \mathrm{e}^{-\frac{\mu \eta}{2 \mathbf{S}(H, \mu)}|x-y|^{\epsilon}}\left\langle\mathfrak{e}_{\mathbf{x}},\left((H-u)^{2}+\eta^{2}\right)^{-1} \mathfrak{e}_{\mathbf{x}}\right\rangle^{1 / 2}\left\langle\mathfrak{e}_{\mathbf{y}},\left((H-u)^{2}+\eta^{2}\right)^{-1} \mathfrak{e}_{\mathbf{y}}\right\rangle^{1 / 2}
\end{aligned}
$$

for $\mathbf{x}=(x, \mathrm{~s}, v), \mathbf{y}=(y, \mathrm{t}, w) \in \mathbb{X}_{\infty}, u \in \mathbb{R}$ and $\eta \in(0,2 \mathbf{S}(H, \mu)]$. On the other hand, at fixed $\alpha \in[0, \beta]$ and $\beta \in \mathbb{R}^{+}$the function on the stripe

$$
\mathbb{R}+\frac{\pi i}{2 \beta}[-1,1] \subset \mathbb{C}
$$


defined by

$$
G(z) \doteq \frac{\mathrm{e}^{\alpha z}}{1+\mathrm{e}^{\beta z}}
$$

is analytic and uniformly bounded by 1 , because

$$
\begin{aligned}
& \sup _{x \in \mathbb{R}, y \in[-\pi / 2 \beta, \pi / 2 \beta]}|G(x+i y)|=\sup _{x \in \mathbb{R}, y \in[-\pi / 2 \beta, \pi / 2 \beta]} \frac{\mathrm{e}^{\alpha x}}{\left(1+2 \mathrm{e}^{\beta x} \cos \beta y+\mathrm{e}^{2 \beta x}\right)^{1 / 2}} \\
& \leq \sup _{x \in \mathbb{R}} \frac{\mathrm{e}^{\alpha x}}{\left(1+\mathrm{e}^{2 \beta x}\right)^{1 / 2}} \leq 1
\end{aligned}
$$

for $\alpha \in[0, \beta]$ and $\beta \in \mathbb{R}^{+}$. Using Cauchy's integral formula and some translation by $\pm i \eta$, we write this function as

$$
\begin{aligned}
G(E) & =\frac{1}{2 \pi i} \int_{\mathbb{R}}\left(\frac{G(u-i \eta)}{u-i \eta-E}-\frac{G(u+i \eta)}{u+i \eta-E}\right) \mathrm{d} u \\
& =\frac{\eta}{\pi} \int_{\mathbb{R}} \frac{G(u-i \eta)+G(u+i \eta)}{(E-u)^{2}+\eta^{2}} \mathrm{~d} u-\frac{2 \eta}{\pi} \int_{\mathbb{R}} \frac{G(u)}{(E-u)^{2}+4 \eta^{2}} \mathrm{~d} u
\end{aligned}
$$

for all $E \in \mathbb{R}$ and $\eta \in(0, \pi /(2 \beta)]$. By spectral calculus, together with (104)-(105) and the CauchySchwarz inequality, it follows that

$$
\sup _{\alpha \in[0, \beta]}\left|\left\langle\mathfrak{e}_{\mathbf{x}}, \frac{\mathrm{e}^{\alpha H}}{1+\mathrm{e}^{\beta H}} \mathfrak{e}_{\mathbf{y}}\right\rangle_{\mathcal{H}_{\infty}}\right| \leq 48 \exp \left(-\mu \min \left\{1, \frac{\pi}{4 \beta \mathbf{S}(H, \mu)}\right\}|x-y|^{\epsilon}\right)
$$

for all $\mathbf{x}=(x, \mathrm{~s}, v), \mathbf{y}=(y, \mathrm{t}, w) \in \mathbb{X}_{\infty}, \mu \in \mathbb{R}_{0}^{+}$, and $\epsilon \in(0,1]$. This in turn implies the assertion. In particular, for $v=\beta^{-1} \mu_{0} \in \mathbb{R}^{+}$and fixed $\epsilon \in(0,1]$, where $\mu_{0} \in \mathbb{R}^{+}$is some constant such that $8 \mathbf{S}\left(H, \mu_{0}\right) \leq \pi$ (which always exists, because of (103)), by taking $\mu=2 v$ and using (103), one gets that

$$
\mathbf{D}_{H, \beta, v, \epsilon} \leq 96|\mathrm{~S}| \sum_{x \in \mathbb{Z}^{d}} \mathrm{e}^{-\mu_{0} \beta^{-1}|x|^{\epsilon}}=\mathcal{O}\left((\beta+1)^{d \epsilon^{-1}}\right)
$$

\subsubsection{Summability of Covariances - General Bound}

We consider the following condition:

\section{Condition 5.9}

There is a sequence $\mathcal{P} \doteq\left\{P_{L}\right\}_{L \in \mathbb{R}_{0}^{+}}$of basis projections associated with $\left(\mathcal{H}_{L}, \mathfrak{A}_{L}\right)$, diagonalizing $H_{L}$, such that:

(a) $P_{L}$ strongly converges, as $L \rightarrow \infty$, to an orthogonal projection $P_{\infty} \in \mathcal{B}\left(\mathcal{H}_{\infty}\right)$.

(b) The limit projection $P_{\infty}$ satisfies

$$
\mathbf{D}_{\mathcal{P}, v, \epsilon} \doteq \sup _{\mathbf{x}_{1}=\left(x_{1}, \mathrm{~s}_{1}, v_{1}\right) \in \mathbb{X}_{\infty}} \sum_{\mathbf{x}_{2}=\left(x_{2}, \mathbf{s}_{2}, v_{2}\right) \in \mathbb{X}_{\infty}} \mathrm{e}^{v\left|x_{1}-x_{2}\right|^{\epsilon}}\left|\left\langle\mathfrak{e}_{\mathbf{x}_{1}}, P_{\infty} \mathfrak{e}_{\mathbf{x}_{2}}\right\rangle_{\mathcal{H}_{\infty}}\right|<\infty
$$

for some fixed $\epsilon \in(0,1]$ and $v \in \mathbb{R}_{0}^{+}$.

This condition is trivially fulfilled for all gauge-invariant self-dual Hamiltonians, that is, self-dual Hamiltonians $H_{\infty}$ satisfying

$$
\left\langle\mathfrak{e}_{(x, \mathrm{~s}, v)}, H_{\infty} \mathfrak{e}_{(y, \mathrm{t}, v)}\right\rangle=0, \quad x, y \in \mathbb{Z}^{d}, \mathrm{~s}, \mathrm{t} \in \mathrm{S}, v \in\{+,-\} .
$$


In this case, for any $L \in \mathbb{R}_{0}^{+}$, one can choose the sequence $\left\{P_{L}\right\}_{L \in \mathbb{R}_{0}^{+}}$of basis projections defined by

$$
P_{L} \mathfrak{e}_{(x, \mathrm{~s}, v)}=\delta_{v,-} \mathfrak{e}_{(x, \mathrm{~s}, v)}, \quad(x, \mathrm{~s}, v) \in \Lambda_{L} \times \mathrm{S} \times\{+,-\}
$$

where $\left\{\mathfrak{e}_{\mathbf{x}}\right\}_{\mathbf{x} \in \mathbb{X}_{\infty}}$ is the orthonormal basis defined by (101). This obviously yields $\mathbf{D}_{\mathcal{P}}=1$.

Condition 5.9 (a) is convenient because it allows us to rewrite the three limits of the decay parameter

$$
\begin{aligned}
\boldsymbol{\omega}_{H_{\infty}, \mathcal{P}, v, \epsilon, \boldsymbol{I}} \doteq & \limsup _{L_{\mathrm{i}} \rightarrow \infty} \limsup _{L_{\mathrm{f}} \rightarrow \infty} \lim _{n \rightarrow \infty} \sup _{k_{1} \in\left\{0, \ldots, n_{\beta}-1\right\} \mathbf{x}_{1}=\left(x_{1}, \mathrm{~s}_{1}, v_{1}\right) \in \mathbb{X}_{L_{\mathrm{i}}}} \sup \\
& \left\{\beta n^{-1} \sum_{k_{2}=0}^{n_{\beta}-1} \sum_{\mathbf{x}_{2}=\left(x_{2}, \mathrm{~s}_{2}, v_{2}\right) \in \mathbb{X}_{L_{\mathrm{i}}}} \mathrm{e}^{\beth \tilde{\alpha}\left(\beta^{-1} k_{1}, \beta^{-1} k_{2}\right)+v\left|x_{1}-x_{2}\right|^{\epsilon}}\left|\left\langle\mathfrak{e}_{\mathbf{x}_{1}}^{\left(k_{1}\right)}, C_{H_{L_{\mathrm{f}}}, P_{L_{\mathrm{f}}}}^{(n)} \mathfrak{e}_{\mathbf{x}_{2}}^{\left(k_{2}\right)}\right\rangle_{\mathfrak{H}^{\left(n_{\beta}\right)}}\right|\right\}
\end{aligned}
$$

in a more tractable form, where $\epsilon \in(0,1], v, \beth \in \mathbb{R}_{0}^{+}, \tilde{\alpha}$ is the pseudometric on $[0, \beta+1]$ defined by

$$
\tilde{\alpha}\left(u_{1}, u_{2}\right) \doteq \min \left\{\alpha\left(u_{1}, u_{2}\right), \beta-\alpha\left(u_{1}, u_{2}\right)\right\}, \quad u_{1}, u_{2} \in[0, \beta+1],
$$

with

$\alpha\left(u_{1}, u_{2}\right) \doteq\left[\min \left\{\beta-\min \left\{u_{1}, u_{2}\right\}, \max \left\{u_{1}, u_{2}\right\}-\min \left\{u_{1}, u_{2}\right\}\right\}\right]_{+} \in[0, \beta], \quad u_{1}, u_{2} \in[0, \beta+1)$

Recall that $C_{H_{L_{\mathrm{f}}}, P_{L_{\mathrm{f}}}}^{(n)}$ is the covariance (76) for $\mathcal{H}=\mathcal{H}_{L_{\mathrm{f}}}$ and $n_{\beta} \doteq n+\lfloor n / \beta\rfloor$. Similarly to Equations (96) and (97), define the function

$$
F_{u_{1}, u_{2}}(H, P) \doteq \begin{cases}P \frac{\mathrm{e}^{-\alpha\left(u_{1}, u_{2}\right) H}}{1+\mathrm{e}^{-\beta H}} P+P^{\perp} \frac{\mathrm{e}^{-\alpha\left(u_{1}, u_{2}\right) H}}{1+\mathrm{e}^{-\beta H}} P^{\perp} & \text { for } u_{1}<u_{2}, \\ -P \frac{\mathrm{e}^{\alpha\left(u_{1}, u_{2}\right) H}}{1+\mathrm{e}^{\beta H}} P-P^{\perp} \frac{\mathrm{e}^{\alpha\left(u_{1}, u_{2}\right) H}}{1+\mathrm{e}^{\beta H}} P^{\perp} & \text { for } u_{1} \geq u_{2}\end{cases}
$$

for any $\beta \in \mathbb{R}^{+}, u_{1}, u_{2} \in[0, \beta+1), H \in \mathcal{B}(\mathcal{H})$, and orthogonal projection $P$. Then, one gets the following expression for the decay parameter $\boldsymbol{\omega}_{H_{\infty}, \mathcal{P}, v, \epsilon, 0}$ :

\section{Lemma 5.10 (Explicit expression of the decay parameter)}

Fix $d \in \mathbb{N}, \beta \in \mathbb{R}^{+}, \epsilon \in(0,1]$ and $v, \rrbracket \in \mathbb{R}_{0}^{+}$. Let $H_{\infty} \in \mathcal{B}\left(\mathcal{H}_{\infty}\right)$ be any self-dual Hamiltonian on $\left(\mathcal{H}_{\infty}, \mathfrak{A}_{\infty}\right)$. If Condition 5.9 (a) holds true, then

$$
\begin{aligned}
\boldsymbol{\omega}_{H_{\infty}, \mathcal{P}, v, \epsilon, \mathbf{I}}=\sup _{u_{1} \in[0, \beta+1)} \sup _{\mathbf{x}_{1}=\left(x_{1}, \mathrm{~s}_{1}, v_{1}\right) \in \mathbb{X}_{\infty}} & \sum_{\mathbf{x}_{2}=\left(x_{2}, \mathrm{~s}_{2}, v_{2}\right) \in \mathbb{X}_{\infty}} \mathrm{e}^{v\left|x_{1}-x_{2}\right|^{\epsilon}} \\
& \times \int_{[0, \beta+1)} \mathrm{e}^{\mathrm{I} \tilde{\alpha}\left(u_{1}, u_{2}\right)}\left|\left\langle\mathfrak{e}_{\mathbf{x}_{1}}, F_{u_{1}, u_{2}}\left(H_{\infty}, P_{\infty}\right) \mathfrak{e}_{\mathbf{x}_{2}}\right\rangle_{\mathcal{H}_{\infty}}\right| \mathrm{d} u_{2} .
\end{aligned}
$$

Proof. Fix all parameters of the lemma. Explicit computations using the spectral theorem and Inequality (95) show that, for any $\beta \in \mathbb{R}^{+}, n>\beta\left\|H_{\infty}\right\|_{\mathcal{B}\left(\mathcal{H}_{\infty}\right)}, L_{\mathrm{f}} \in \mathbb{R}_{0}^{+} \cup\{\infty\}$ and $\alpha \in[0, \beta]$,

$$
\begin{aligned}
\left\|\frac{\mathrm{e}^{ \pm \alpha H_{L_{\mathrm{f}}}}}{1+\mathrm{e}^{ \pm \beta H_{L_{\mathrm{f}}}}}-\frac{\mathrm{e}^{ \pm \alpha H_{L_{\mathrm{f}}}^{(n)}}}{1+\mathrm{e}^{ \pm \beta H_{L_{\mathrm{f}}}^{(n)}}}\right\|_{\mathcal{B}\left(\mathcal{H}_{\infty}\right)} & \leq \sup _{|\lambda| \leq\left\|H_{L_{\mathrm{f}}}\right\|_{\mathcal{B}\left(\mathcal{H}_{\infty}\right)}}\left|\frac{\mathrm{e}^{ \pm \alpha \lambda}}{1+\mathrm{e}^{ \pm \beta \lambda}}-\frac{\mathrm{e}^{ \pm \frac{\alpha \beta^{-1} n}{2}\left(\ln \left(\frac{1+n^{-1} \beta \lambda}{1-n^{-1} \beta \lambda}\right)\right.}}{1+\mathrm{e}^{ \pm \frac{n}{2}\left(\ln \left(\frac{1+n^{-1} \beta \lambda}{1-n^{-1} \beta \lambda}\right)\right)}}\right| \\
& \leq 2 \beta\left\|H_{L_{\mathrm{f}}}-H_{L_{\mathrm{f}}}^{(n)}\right\|_{\mathcal{B}\left(\mathcal{H}_{\infty}\right)} \leq D n^{-2} \beta^{3}\left\|H_{\infty}\right\|_{\mathcal{B}\left(\mathcal{H}_{\infty}\right)}^{3},
\end{aligned}
$$


for some finite constant $D$. It follows that

$$
\begin{aligned}
\boldsymbol{\omega}_{H_{\infty}, \mathcal{P}, v, \epsilon, \boldsymbol{I}}=\limsup _{L_{\mathrm{i}} \rightarrow \infty} \limsup _{L_{\mathrm{f}} \rightarrow \infty} \sup _{u_{1} \in[0, \beta+1)} \sup _{\mathbf{x}_{1} \in \mathbb{X}_{L_{\mathrm{i}}}} \\
\left.\qquad \sum_{\mathbf{x}_{2} \in \mathbb{X}_{L_{\mathrm{i}}}} \mathrm{e}^{v\left|x_{1}-x_{2}\right|^{\epsilon}} \int_{[0, \beta+1)} \mathrm{e}^{\beth \tilde{\alpha}\left(u_{1}, u_{2}\right)}\left|\left\langle\mathfrak{e}_{\mathbf{x}_{1}}, F_{u_{1}, u_{2}}\left(H_{L_{\mathrm{f}}}, P_{L_{\mathrm{f}}}\right) \mathfrak{e}_{\mathbf{x}_{2}}\right\rangle_{\mathcal{H}_{\infty}}\right| \mathrm{d} u_{2}\right\}
\end{aligned}
$$

for $\epsilon \in(0,1]$ and $v, \beth \in \mathbb{R}_{0}^{+}$. By Condition 5.9 (a), $P_{L_{\mathrm{f}}}$ strongly converges, as $L_{\mathrm{f}} \rightarrow \infty$, to an orthogonal projection $P_{\infty}$. Therefore, because the set $\mathbb{X}_{L_{\mathrm{i}}}$ is finite and the family

$$
E \mapsto \int_{[0, \beta+1)} \frac{\mathrm{e}^{\beth \tilde{\alpha}\left(u_{1}, u_{2}\right) \pm E \alpha\left(u_{1}, u_{2}\right)}}{1+\mathrm{e}^{ \pm \beta E}} \mathrm{~d} u_{2}, \quad u_{1} \in[0, \beta+1),
$$

of functions is uniformly equicontinuous on compact subsets of $\mathbb{R}$, we deduce from the last equality that

$$
\begin{aligned}
\boldsymbol{\omega}_{H_{\infty}, \mathcal{P}, v, \epsilon, \beth}=\limsup _{L_{\mathrm{i}} \rightarrow \infty} & \sup _{u_{1} \in[0, \beta+1)} \sup _{\mathbf{x}_{1} \in \mathbb{X}_{L_{\mathrm{i}}}} \\
& \left\{\sum_{\mathbf{x}_{2} \in \mathbb{X}_{L_{\mathrm{i}}}} \mathrm{e}^{v\left|x_{1}-x_{2}\right|^{\epsilon}} \int_{[0, \beta+1)} \mathrm{e}^{\mathbf{I} \tilde{\alpha}\left(u_{1}, u_{2}\right)}\left|\left\langle\mathfrak{e}_{\mathbf{x}_{1}}, F_{u_{1}, u_{2}}\left(H_{\infty}, P_{\infty}\right) \mathfrak{e}_{\mathbf{x}_{2}}\right\rangle_{\mathcal{H}_{\infty}}\right| \mathrm{d} u_{2}\right\} .
\end{aligned}
$$

Finally, by using approximate maximizers of the suprema over $u_{1} \in[0, \beta+1)$ and $\mathbf{x}_{1} \in \mathbb{X}_{\infty}$, and the monotone convergence, the lemma follows.

Now we are in a position to prove the summability of the covariance:

\section{Theorem 5.11 (Summability of the covariance)}

Fix a (finite) spin set $\mathrm{S}, d \in \mathbb{N}$ and let $H_{\infty} \in \mathcal{B}\left(\mathcal{H}_{\infty}\right)$ be any self-dual Hamiltonian on $\left(\mathcal{H}_{\infty}, \mathfrak{A}_{\infty}\right)$. Then, under Condition 5.9, for any $\beta \in \mathbb{R}^{+}, \epsilon \in(0,1]$ and $v \in \mathbb{R}_{0}^{+}$,

$$
\boldsymbol{\omega}_{H_{\infty}, \mathcal{P}, v, \epsilon, 0} \leq 2\left(\mathbf{D}_{\mathcal{P}, v, \epsilon}+1\right)^{2} \mathbf{D}_{H, \beta, v, \epsilon}(\beta+1) .
$$

Proof. The theorem is a consequence of Condition 5.9, Lemmata 5.8 and 5.10, and Equation (108) together with straightforward computations.

By Lemma 5.8, we observe from this theorem that

$$
\boldsymbol{\omega}_{H_{\infty}, \mathcal{P}, v, \epsilon, 0}=\mathcal{O}\left((\beta+1)^{d \epsilon^{-1}+1}\right),
$$

for all self-dual Hamiltonians $H_{\infty} \in \mathcal{B}\left(\mathcal{H}_{\infty}\right)$ as soon as $\mathbf{D}_{\mathcal{P}, v, \epsilon}<\infty$ and $4 \beta \mathbf{S}(H, \mu) v<\pi \mu$ for some $\mu>v$ and $\epsilon \in(0,1]$. Recall that if $H_{\infty}$ is gauge-invariant then one can take $\mathbf{D}_{\mathcal{P}, v, \epsilon}=1$. The scaling (109) with respect to the inverse temperature $\beta \in \mathbb{R}^{+}$is exactly the same one obtained from the Fourier analysis of the two-point Green function of translation-invariant, free fermi systems at positive density. Our result shows that this estimate does not depend on translation invariance.

\subsubsection{Summability of Covariances - Gapped Case}

Estimate (109) does not allow for the control of the decay parameter $\boldsymbol{\omega}_{H_{\infty}, \mathcal{P}, v, \epsilon, 0}(106)$ in the zerotemperature limit, i.e., when $\beta \rightarrow \infty$. However, in the special case of gapped self-dual Hamiltonians, that is, self-dual Hamiltonians $H_{\infty}$ satisfying

$$
\mathfrak{g}_{H_{\infty}} \doteq \inf \left\{\varepsilon>0:[-\varepsilon, \varepsilon] \cap \operatorname{spec}\left(H_{\infty}\right) \neq \emptyset\right\}>0,
$$

we can uniformly bound $\boldsymbol{\omega}_{H_{\infty}, \mathcal{P}, v, \epsilon, 0}$ at arbitrarily large $\beta \gg 1$, for a natural choice of sequence $\mathcal{P}=\left\{P_{L}\right\}_{L \in \mathbb{R}_{0}^{+}}$of basis projections associated with $\left(\mathcal{H}_{L}, \mathfrak{A}_{L}\right)$ and diagonalizing $H_{L}, L \in \mathbb{R}_{0}^{+}$. To demonstrate this, we need to adapt Lemma 5.8 to this gapped situation: 


\section{Lemma 5.12 (Summability of the Fermi distribution - gapped case)}

Fix a (finite) spin set $\mathrm{S}, d \in \mathbb{N}, \beta \in \mathbb{R}^{+}, \epsilon \in(0,1], v, \beth \in \mathbb{R}_{0}^{+}$and $H=H^{*} \in \mathcal{B}\left(\mathcal{H}_{\infty}\right)$ such that $\mathfrak{g}_{H}>0$. Then,

$$
\begin{aligned}
& \sup _{u_{1} \in[0, \beta+1)} \sup _{\mathbf{x}_{1}=\left(x_{1}, \mathrm{~s}_{1}, v_{1}\right) \in \mathbb{X}_{\infty}}\left\{\sum_{\mathbf{x}_{2}=\left(x_{2}, \mathrm{~s}_{2}, v_{2}\right) \in \mathbb{X}_{\infty}} \mathrm{e}^{v\left|x_{1}-x_{2}\right|^{\epsilon}}\right. \\
& \left.\times \int_{[0, \beta+1)} \mathrm{e}^{\mathrm{J} \tilde{\alpha}\left(u_{1}, u_{2}\right)}\left|\left\langle\mathfrak{e}_{\mathbf{x}_{1}}, F_{u_{1}, u_{2}}(H, \mathbf{1}[H>0]) \mathfrak{e}_{\mathbf{x}_{2}}\right\rangle_{\mathcal{H}_{\infty}}\right| \mathrm{d} u_{2}\right\} \\
& \leq 152|\mathrm{~S}| \sup _{u_{1} \in[0, \beta+1)} \inf _{\mu \in \mathbb{R}_{0}^{+}} \inf _{\epsilon \in(0,1]} \sum_{x \in \mathbb{Z}^{d}} \mathrm{e}^{\left(v-\mu \min \left\{1, \frac{\mathfrak{g}_{H}}{4 \mathbf{S}(H, \mu)}\right\}\right)|x|^{\epsilon}} \int_{0}^{\beta+1} \frac{\mathrm{e}^{\left(\boldsymbol{J}-\frac{\mathfrak{g}_{H}}{2}\right) \tilde{\alpha}\left(u_{1}, u_{2}\right)}}{1-\mathrm{e}^{-\frac{\beta \mathfrak{g}_{H}}{2}}} \mathrm{~d} u_{2},
\end{aligned}
$$

where we recall that $\tilde{\alpha}$ is the pseudometric on $[0, \beta+1]$ defined by (107).

Proof. Fix all parameters of the lemma. By Inequality (103), Theorem 5.6 implies the bound

$$
\left|\left\langle\mathfrak{e}_{\mathbf{x}},(z-H)^{-1} \mathfrak{e}_{\mathbf{y}}\right\rangle\right| \leq 4 \mathfrak{g}_{H}^{-1} \exp \left(-\mu \min \left\{1, \frac{\mathfrak{g}_{H}}{4 \mathbf{S}(H, \mu)}\right\}|x-y|^{\epsilon}\right)
$$

for any $\mathbf{x}=(x, \mathrm{~s}, v), \mathbf{y}=(y, \mathrm{t}, w) \in \mathbb{X}_{\infty}$ and $z \in \mathbb{C}$ such that $\Delta(H, z) \geq \mathfrak{g}_{H} / 2>0$. On the other hand, for every $\eta \in\left(0, \mathfrak{g}_{H} / 2\right]$, the function defined by

$$
G(z) \doteq \frac{\mathrm{e}^{-\alpha z}}{1+\mathrm{e}^{-\beta z}}, \quad z \in\left(\mathbb{R}_{0}^{+}+\eta+i \eta[-1,1]\right)
$$

is analytic and uniformly bounded by $\mathrm{e}^{-\alpha \eta}\left(1-\mathrm{e}^{-\beta \eta}\right)^{-1}$. Similar to (105), we again use Cauchy's integral formula to write, for all real $E \in \mathbb{R} \backslash\{\eta\}$,

$$
\mathbf{1}[E>\eta] G(E)=\frac{1}{2 \pi i} \int_{\eta}^{\infty}\left(\frac{G(u-i \eta)}{u-E-i \eta}-\frac{G(u+i \eta)}{u-E+i \eta}\right) \mathrm{d} u-\frac{1}{2 \pi} \int_{-\eta}^{\eta} \frac{G(\eta+i u)}{\eta-E+i u} \mathrm{~d} u,
$$

which yields

$$
\begin{aligned}
\mathbf{1}[E>\eta] G(E)= & \frac{\eta}{\pi} \int_{\eta}^{\infty} \frac{G(u-i \eta)+G(u+i \eta)}{(u-E)^{2}+\eta^{2}} \mathrm{~d} u-\frac{2 \eta}{\pi} \int_{\eta}^{\infty} \frac{G(u)}{(u-E)^{2}+4 \eta^{2}} \mathrm{~d} u \\
& +\frac{1}{2 \pi} \int_{0}^{\eta} \frac{G(\eta-i u)}{\eta-i u-E+2 i \eta} \mathrm{d} u+\frac{1}{2 \pi} \int_{0}^{\eta} \frac{G(\eta+i u)}{\eta+i u-E-2 i \eta} \mathrm{d} u \\
& -\frac{1}{2 \pi} \int_{-\eta}^{\eta} \frac{G(\eta+i u)}{\eta-E+i u} \mathrm{~d} u .
\end{aligned}
$$

$E \mapsto \mathbf{1}[E>\eta]$ is the characteristic function of the set $(\eta, \infty)$. By spectral calculus, together with the last equality, Inequalities (104) and (111) and the Cauchy-Schwarz inequality, it follows that

$$
\left|\left\langle\mathfrak{e}_{\mathbf{x}}, \mathbf{1}[H>0] \frac{\mathrm{e}^{-\alpha H}}{1+\mathrm{e}^{-\beta H}} \mathbf{1}[H>0] \mathfrak{e}_{\mathbf{y}}\right\rangle_{\mathcal{H}_{\infty}}\right| \leq \frac{38 \mathrm{e}^{-\frac{\alpha \mathfrak{g}_{H}}{2}}}{1-\mathrm{e}^{-\frac{\beta \mathfrak{g}_{H}}{2}}} \mathrm{e}^{-\mu \min \left\{1, \frac{\mathfrak{g}_{H}}{4 \mathbf{S}(H, \mu)}\right\}|x-y|^{\epsilon}}
$$

for all $\mathbf{x}=(x, \mathrm{~s}, v), \mathbf{y}=(y, \mathrm{t}, w) \in \mathbb{X}_{\infty}, \mu \in \mathbb{R}_{0}^{+}, \epsilon \in(0,1]$ and $\alpha \in[0, \beta)$. In the same way,

$$
\left|\left\langle\mathfrak{e}_{\mathbf{x}}, \mathbf{1}[H>0] \frac{\mathrm{e}^{\alpha H}}{1+\mathrm{e}^{\beta H}} \mathbf{1}[H>0] \mathfrak{e}_{\mathbf{y}}\right\rangle_{\mathcal{H}_{\infty}}\right| \leq \frac{38 \mathrm{e}^{\frac{\alpha \mathfrak{g}_{H}}{2}}}{\mathrm{e}^{\frac{\beta \mathfrak{g}_{H}}{2}}-1} \mathrm{e}^{-\mu \min \left\{1, \frac{\mathfrak{g}_{H}}{4 \mathbf{S}(H, \mu)}\right\}|x-y|^{\epsilon}}
$$

for all $\mathbf{x}=(x, \mathrm{~s}, v), \mathbf{y}=(y, \mathrm{t}, w) \in \mathbb{X}_{\infty}, \mu \in \mathbb{R}_{0}^{+}, \epsilon \in(0,1]$ and $\alpha \in[0, \beta)$. By (108), this in turn implies the assertion.

We are now in a position to prove the uniform summability of the covariance with respect to the inverse temperature $\beta \in \mathbb{R}^{+}$, in the special case of gapped Hamiltonians: 


\section{Theorem 5.13 (Summability of the covariance - gapped case)}

Fix a (finite) spin set $\mathrm{S}, d \in \mathbb{N}, \in \in(0,1], v, \rrbracket \in \mathbb{R}_{0}^{+}$and let $H_{\infty} \in \mathcal{B}\left(\mathcal{H}_{\infty}\right)$ be any self-dual Hamiltonian on $\left(\mathcal{H}_{\infty}, \mathfrak{A}_{\infty}\right)$ such that $\mathfrak{g}_{H_{\infty}}>2$ J. Then, there is a sequence $\mathcal{P} \doteq\left\{P_{L}\right\}_{L \in \mathbb{R}^{+}}$of basis projections associated with $\left(\mathcal{H}_{L}, \mathfrak{A}_{L}\right)$, diagonalizing $H_{L}$, such that, for any $\beta \in \mathbb{R}^{+}$,

$$
\boldsymbol{\omega}_{H_{\infty}, \mathcal{P}, v, \epsilon, \mathrm{I}} \leq 152|\mathrm{~S}| \sup _{u_{1} \in[0, \beta+1)} \inf _{\mu \in \mathbb{R}_{0}^{+}} \inf _{\epsilon \in(0,1]} \sum_{x \in \mathbb{Z}^{d}} \mathrm{e}^{\left(v-\mu \min \left\{1, \frac{\mathfrak{g}_{H \infty}}{4 \mathbf{S}\left(H_{\infty}, \mu\right)}\right\}\right)|x|^{\epsilon}} \int_{0}^{\beta+1} \frac{\mathrm{e}^{\left(\boldsymbol{I}-\frac{\mathfrak{g}_{H_{\infty}}}{2}\right) \tilde{\alpha}\left(u_{1}, u_{2}\right)}}{1-\mathrm{e}^{-\frac{\beta \mathfrak{g}_{H}}{2}} \mathrm{~d} u_{2}} .
$$

In particular, if $4 \mathbf{S}\left(H_{\infty}, \mu\right) v<\mathfrak{g}_{H_{\infty}} \mu$ for some $\mu>v$ and $\mathfrak{g}_{H_{\infty}}>2$ I then

$$
\boldsymbol{\omega}_{H_{\infty}, \mathcal{P}, v, \epsilon, \mathrm{I}}=\mathcal{O}\left(\left(\left(\mathfrak{g}_{H_{\infty}} / 2-\mathfrak{I}\right)^{-1}+1\right)\left(\mathfrak{g}_{H_{\infty}}^{-1}+1\right)^{d}\right) .
$$

Proof. Fix all parameters of the theorem. Since $H_{\infty} \in \mathcal{B}\left(\mathcal{H}_{\infty}\right)$ is a self-dual Hamiltonian on $\left(\mathcal{H}_{\infty}, \mathfrak{A}_{\infty}\right)$, note again that $H_{L}$, as defined by (102) for any $L \in \mathbb{R}_{0}^{+}$, is a self-dual Hamiltonian on $\left(\mathcal{H}_{L}, \mathfrak{A}_{L}\right)$. Therefore, for all $L \in \mathbb{R}_{0}^{+}$,

$$
\mathfrak{A}_{L} \mathbf{1}\left[ \pm H_{L}>0\right] \mathfrak{A}_{L}=\mathbf{1}\left[\mp H_{L}>0\right] \quad \text { and } \quad \mathfrak{A}_{L} \mathbf{1}\left[H_{L}=0\right] \mathfrak{A}_{L}=\mathbf{1}\left[H_{L}=0\right] .
$$

Because the dimension of $\mathcal{H}_{L}$ is even, for any $L \in \mathbb{R}_{0}^{+}$, by (112), the kernel $\operatorname{ker} H_{L}$ also has even dimension. Hence, there is a basis projection $P_{L}^{(0)}$ associated with $\left(\operatorname{ker} H_{L},\left.\mathfrak{A}_{L}\right|_{\operatorname{ker} H_{L}}\right)$ for any $L \in \mathbb{R}_{0}^{+}$, by [A68, Lemma 3.3]. In particular, from (112),

$$
P_{L} \doteq P_{L}^{(0)}+\mathbf{1}\left[H_{L}>0\right], \quad L \in \mathbb{R}_{0}^{+},
$$

is a basis projection diagonalizing $H_{L}$. Let $\chi: \mathbb{R} \rightarrow \mathbb{R}_{0}^{+}$be any continuous function, whose support lies on the interval $\left(-\mathfrak{g}_{H_{\infty}}, \mathfrak{g}_{H_{\infty}}\right)$, such that $\chi(0)=1$. Then,

$$
0 \leq P_{L}^{(0)} \leq \chi\left(H_{L}\right), \quad L \in \mathbb{R}_{0}^{+} .
$$

Noting that, as $L \rightarrow \infty, H_{L}$ strongly converges to $H_{\infty}$, we infer from the gap assumption (110) on the spectrum of $H_{\infty}$ that $\chi\left(H_{L}\right)$ and, hence, $P_{L}^{(0)}$ strongly tend to 0 when $L \rightarrow \infty$. Therefore, $P_{L}$ strongly converges to $1\left[H_{\infty}>0\right]$, as $L \rightarrow \infty$, and Condition 5.9 (a) is satisfied. Applying now Lemmata 5.10 and 5.12 together with the asymptotics

$$
\int_{0}^{\beta+1} \frac{\mathrm{e}^{\left(\mathrm{J}-\frac{\mathfrak{g}_{H}}{2}\right) \tilde{\alpha}\left(u_{1}, u_{2}\right)}}{1-\mathrm{e}^{-\frac{\beta \mathfrak{g}_{H}}{2}}} \mathrm{~d} u_{2}=\mathcal{O}\left(\left(\mathfrak{g}_{H_{\infty}} / 2-\mathfrak{I}\right)^{-1}+1\right)
$$

uniformly for $\beta \geq D>0$ and $u_{1} \in\left[0, \beta+1\right.$ ) (when $\mathfrak{g}_{H_{\infty}}>2 \beth$ ), the assertion follows.

\section{CAR Algebra and Second Quantization}

\subsection{CAR Algebra}

In Section 6, for simplicity, $\mathfrak{h}$, the so-called one-particle Hilbert space, is finite-dimensional. Then, given such an $\mathfrak{h}$, the associated CAR algebra is defined as follows:

\section{Definition 6.1 (CAR algebra)}

The CAR algebra $\operatorname{CAR}(\mathfrak{h}) \equiv(\operatorname{CAR}(\mathfrak{h}),+, \cdot, *)$ is the $C^{*}$-algebra generated by a unit 1 and a family $\{a(\varphi)\}_{\varphi \in \mathfrak{h}}$ of elements satisfying Conditions (a)-(b):

(a) The map $\varphi \mapsto a(\varphi)^{*}$ is (complex) linear.

(b) The family $\{a(\varphi)\}_{\varphi \in \mathfrak{h}}$ satisfies the CAR: For all $\varphi_{1}, \varphi_{2} \in \mathfrak{h}$,

$$
a\left(\varphi_{1}\right) a\left(\varphi_{2}\right)+a\left(\varphi_{2}\right) a\left(\varphi_{1}\right)=0, \quad a\left(\varphi_{1}\right) a\left(\varphi_{2}\right)^{*}+a\left(\varphi_{2}\right)^{*} a\left(\varphi_{1}\right)=\left\langle\varphi_{1}, \varphi_{2}\right\rangle_{\mathfrak{h}} \mathbf{l} .
$$




\section{Remark 6.2}

By the CAR(113), the antilinear map $\varphi \mapsto a(\varphi)$ is injective and isometric. In particular, $\|a(\varphi)\|_{\mathrm{CAR}(\mathfrak{h})}=$ $\|\varphi\|_{\mathfrak{h}}$ for any $\varphi \in \mathfrak{h}$.

Strictly speaking, the above conditions only define $\operatorname{CAR}(\mathfrak{h})$ up to an isomorphism of $C^{*}$-algebra [BR96b, Theorem 5.2.5]. In particular, if $\{\tilde{a}(\varphi)\}_{\varphi \in \mathfrak{h}}$ is a second family of generators of CAR(h) satisfying (113), then there is a $*$-automorphism $\tau$ of $\operatorname{CAR}(\mathfrak{h})$ such that

$$
a(\varphi)=\tau(\tilde{a}(\varphi)), \quad \varphi \in \mathfrak{h} .
$$

See, e.g., [BR96b, Section 5.2.2].

An important example of a $*$-automorphism $\tau=\sigma_{\theta}$ of $\mathrm{CAR}(\mathfrak{h})$ is defined, for any fixed $\theta \in$ $\mathbb{R} /(2 \pi \mathbb{Z})$, by the condition

$$
\sigma_{\theta}(a(\varphi))=\mathrm{e}^{-i \theta} a(\varphi), \quad \varphi \in \mathfrak{h} .
$$

A special role is played by $\sigma_{\pi}$. Elements $A, B \in \operatorname{CAR}(\mathfrak{h})$ satisfying $\sigma_{\pi}(A)=A$ and $\sigma_{\pi}(B)=-B$ are respectively called even and $o d d$, whereas elements $A \in \operatorname{CAR}(\mathfrak{h})$ satisfying $\sigma_{\theta}(A)=A$ for any $\theta \in[0,2 \pi)$ are called gauge invariant. The space of all even elements forms a $C^{*}$-subalgebra, called the even subalgebra of $\operatorname{CAR}(\mathfrak{h})$.

\subsection{From CAR Algebra to Self-Dual CAR Algebra}

The (finite-dimensional) Hilbert space to be used to construct a self-dual CAR algebra is the direct sum

$$
\mathcal{H} \doteq \mathfrak{h} \oplus \mathfrak{h}^{*} .
$$

Compare with Equation (13). The scalar product on $\mathcal{H}$ is

$$
\langle\varphi, \tilde{\varphi}\rangle_{\mathcal{H}} \doteq\left\langle\varphi_{1}, \tilde{\varphi}_{1}\right\rangle_{\mathfrak{h}}+\left\langle\tilde{\varphi}_{2}, \varphi_{2}\right\rangle_{\mathfrak{h}}, \quad \varphi=\left(\varphi_{1}, \varphi_{2}^{*}\right), \tilde{\varphi}=\left(\tilde{\varphi}_{1}, \tilde{\varphi}_{2}^{*}\right) \in \mathcal{H}
$$

Here, $\varphi^{*}$ denotes the element of the dual $\mathfrak{h}^{*}$ of the Hilbert space $\mathfrak{h}$ which is related to $\varphi$ via the Riesz representation. See also Notation 1.3 (i). We define the canonical antiunitary involution $\mathfrak{A}$ of $\mathcal{H}$ by

$$
\mathfrak{A}\left(\varphi_{1}, \varphi_{2}^{*}\right) \doteq\left(\varphi_{2}, \varphi_{1}^{*}\right), \quad \varphi=\left(\varphi_{1}, \varphi_{2}^{*}\right) \in \mathcal{H}
$$

Note that $\varphi^{*}=\mathfrak{A} \varphi$ for any $\varphi \in \mathfrak{h} \subset \mathcal{H}$. Compare with Definition 3.13.

Then, the CAR algebra $\operatorname{CAR}(\mathfrak{h})$ and the self-dual $\operatorname{CAR}$ algebra $\operatorname{sCAR}(\mathcal{H}, \mathfrak{A})$ are the same $C^{*}$ algebra, by defining

$$
\mathrm{B}(\varphi) \equiv \mathrm{B}_{P_{\mathfrak{h}}}(\varphi) \doteq a\left(\varphi_{1}\right)+a\left(\varphi_{2}\right)^{*}, \quad \varphi=\left(\varphi_{1}, \varphi_{2}^{*}\right) \in \mathcal{H}
$$

with $P_{\mathfrak{h}} \in \mathcal{B}(\mathcal{H})$ being the basis projection of $(\mathcal{H}, \mathfrak{A})$ with range $\mathfrak{h}$. Compare (113) with (11), and (117) with (14). The elements $\mathrm{B}(\varphi+\mathfrak{A} \varphi), \varphi \in \mathfrak{h}$, can thus be seen as field operators in the context of CAR algebra.

\subsection{Quadratic Fermionic Hamiltonians as Bilinear Elements}

An important class of even elements of $\mathrm{CAR}(\mathfrak{h})$ in quantum field theory is given by quadratic fermionic Hamiltonians $\mathrm{d} \Gamma(h)+\mathrm{d} \Upsilon(g)$ for $h=h^{*} \in \mathcal{B}(\mathfrak{h})$ and antilinear operators $g=-g^{*}$ on $\mathfrak{h}$, as defined by (4)-(5). Below, we describe the relationship between $\mathrm{d} \Gamma(h), \mathrm{d} \Upsilon(g)$ and the bilinear Hamiltonians from Definition 2.5, starting with the second quantization $\mathrm{d} \Gamma(h)$ of one-particle Hamiltonians $h$ : 
Gauge-invariant case: We lift any operator acting on $\mathfrak{h}$ to an operator acting on $\mathcal{H}$ by using the linear map $\kappa$ from $\mathcal{B}(\mathfrak{h})$ to $\mathcal{B}(\mathcal{H})$ defined by

$$
\kappa(h) \doteq \frac{1}{2}\left(P_{\mathfrak{h}} h P_{\mathfrak{h}}-\mathfrak{A} P_{\mathfrak{h}} h^{*} P_{\mathfrak{h}} \mathfrak{A}\right), \quad h \in \mathcal{B}(\mathfrak{h}) .
$$

Since $P_{\mathfrak{h}}$ is a basis projection, i.e., $\mathfrak{A} P_{\mathfrak{h}} \mathfrak{A}$ is the orthogonal projector on $\mathfrak{h}^{*}$,

$$
\kappa(h)^{*}=\kappa\left(h^{*}\right)=-\mathfrak{A} \kappa(h) \mathfrak{A}, \quad h \in \mathcal{B}(\mathfrak{h}) .
$$

Therefore, by Definition 2.7, $\kappa(h)$ is a self-dual operator on $(\mathcal{H}, \mathfrak{A})$. Compare with (21) and (23).

Second quantizations of the form (4) are, up to constants, bilinear Hamiltonians: Using Equation (117), one verifies that

$$
\mathrm{d} \Gamma(h)=-\langle\mathrm{B}, \kappa(h) \mathrm{B}\rangle+\frac{1}{2} \operatorname{Tr}_{\mathfrak{h}}(h) \mathbf{1}, \quad h=h^{*} \in \mathcal{B}(\mathfrak{h}) .
$$

Compare with Equation (24). Additionally, by (120), for any one-particle Hamiltonian $h \in \mathcal{B}(\mathfrak{h})$, the Gibbs state associated with the quadratic fermionic Hamiltonians $\mathrm{d} \Gamma(h)$ at fixed inverse temperature $\beta \in(0, \infty)$, as given by Lemma 2.10 , is the quasi-free state over $\operatorname{sCAR}(\mathcal{H}, \mathfrak{A})$ whose symbol is $(1+$ $\left.\mathrm{e}^{-\beta \kappa(h)}\right)^{-1}$. In particular, in the one-particle Hilbert space $\mathfrak{h}$, we recover the Fermi-Dirac distribution, at inverse temperature $\beta$, associated with the one-particle Hamiltonian $h$.

Non-gauge-invariant case: Similar to (21) and (118), let

$$
\tilde{\kappa}(g) \doteq \frac{1}{4}\left(P_{\mathfrak{h}}\left(g-g^{*}\right) P_{\mathfrak{h}} \mathfrak{A}+\mathfrak{A} P_{\mathfrak{h}}\left(g^{*}-g\right) P_{\mathfrak{h}}\right) \in \mathcal{B}(\mathcal{H})
$$

for any antilinear operator $g$ on $\mathfrak{h}$. Note that

$$
\tilde{\kappa}(g)^{*}=\tilde{\kappa}(g) \quad \text { and } \quad \tilde{\kappa}(g)=-\mathfrak{A} \tilde{\kappa}(g) \mathfrak{A} .
$$

Therefore, $\tilde{\kappa}(g)$ is a self-dual Hamiltonian on $(\mathcal{H}, \mathfrak{A})$ and, by Definition 2.5,

$$
\mathrm{d} \Upsilon(g)=-\langle\mathrm{B}, \tilde{\kappa}(g) \mathrm{B}\rangle
$$

provided $g=-g^{*}$. By Equation (120), it follows that

$$
\mathrm{d} \Gamma(h)+\mathrm{d} \Upsilon(g)=-\langle\mathrm{B},[\kappa(h)+\tilde{\kappa}(g)] \mathrm{B}\rangle+\frac{1}{2} \operatorname{Tr}_{\mathfrak{h}}(h) \mathbf{l}
$$

for any $h=h^{*} \in \mathcal{B}(\mathfrak{h})$ and antilinear operator $g=-g^{*}$ on $\mathfrak{h}$.

\section{Remark 6.3}

Up to a Bogoliubov *-automorphism (or unitary transformation) and some multiple of the unit 1 , all bilinear Hamiltonians are of the form (120). It is, however, technically advantageous to avoid this unitary transformation while studying the corresponding covariance and correlation functions. See, for instance, (23) and Definition 3.16, as well as Sections 4 and 5.

\subsection{Fock Representation of CAR Algebra}

Note that a CAR $\left(C^{*}\right.$-) algebra can be constructed from any pre-Hilbert space [BR96b, Theorem 5.2.5]. From such a $C^{*}$-algebra, there is always an injective (and thus isometric) homomorphism to the space of bounded operators acting on the corresponding Fock space, by [BR96b, Theorem 5.2.5]. 
This homomorphism is called the Fock representation of the CAR algebra. In the finite dimension situation, this homomorphism is even a $*$-isomorphism of $C^{*}$-algebras.

Here we construct the antisymmetric Fock space from the Grassmann algebra $\wedge^{*} \mathfrak{h}$ (Definition 3.1):

(i): Let $\mathcal{X}$ be a topological vector space. For every $n \in \mathbb{N}$ and $y_{1}, \ldots, y_{n} \in \mathcal{X}$, we define the completely antisymmetric $n$-linear form $y_{1} \wedge \cdots \wedge y_{n}$ from $\left(\mathcal{X}^{*}\right)^{n}$ to $\mathbb{C}$ by

$$
y_{1} \wedge \cdots \wedge y_{n}\left(x_{1}^{*}, \ldots, x_{n}^{*}\right) \doteq \operatorname{det}\left(\left(x_{k}^{*}\left(y_{l}\right)\right)_{k, l=1}^{n}\right), \quad x_{1}^{*}, \ldots, x_{n}^{*} \in \mathcal{X}^{*} .
$$

Compare with (33). Then, similar to (35)-(36) for $\wedge^{*} \mathcal{X}$, one defines the vector space $\wedge \mathcal{X}$.

(ii): For any $n \in \mathbb{N}$, there is a unique linear map $\xi \mapsto\langle\xi, \cdot\rangle_{n}$ from $\wedge^{* n} \mathcal{X}$ to the space $\left(\wedge^{n} \mathcal{X}\right)^{\prime}$ of all linear maps from $\wedge^{n} \mathcal{X}$ to $\mathbb{C}$ such that, for all $x_{1}^{*}, \ldots, x_{n}^{*} \in \mathcal{X}^{*}$,

$$
\left\langle x_{1}^{*} \wedge \cdots \wedge x_{n}^{*}, y_{1} \wedge \cdots \wedge y_{n}\right\rangle_{n}=x_{1}^{*} \wedge \cdots \wedge x_{n}^{*}\left(y_{1}, \ldots, y_{n}\right), \quad y_{1}, \ldots, y_{n} \in \mathcal{X} .
$$

For $n=0,\langle c, d\rangle_{0} \doteq c d$ with $c, d \in \mathbb{C}$. This map is injective for any $n \in \mathbb{N}_{0}$. Then, we denote by $\xi \mapsto\langle\xi, \cdot\rangle$ the unique linear map from $\wedge^{*} \mathcal{X}$ to $(\wedge \mathcal{X})^{\prime}$ such that, for any $n, m \in \mathbb{N}, \xi \in \wedge^{* n} \mathcal{X}$ and $w \in \wedge^{m} \mathcal{X}$

$$
\langle\xi, w\rangle=\left\{\begin{array}{ccc}
\langle\xi, w\rangle_{n} & \text { if } & n=m \\
0 & \text { else } .
\end{array}\right.
$$

(iii): Given two elements $h \in \wedge \mathcal{X}$ and $\xi \in \wedge^{*} \mathcal{X}$, their interior product $\left.h\right\lrcorner \xi \in \wedge^{*} \mathcal{X}$ is uniquely defined by the condition

$$
\langle h\lrcorner \xi, w\rangle=\langle\xi, h \wedge w\rangle, \quad w \in \wedge \mathcal{X} .
$$

(Notice that $\langle\xi, h \wedge(\cdot)\rangle$ is an element of $(\wedge \mathcal{X})^{*}$ of the form $\left.\langle h\lrcorner \xi, \cdot\right\rangle$ for some $\left.h\right\lrcorner \xi \in \wedge^{*} \mathcal{X}$.) For any $h \in \wedge \mathcal{X}$, the map

$$
h\lrcorner: \xi \mapsto h\lrcorner \xi
$$

from $\wedge^{*} \mathcal{X}$ to itself is linear. If $h \in \mathcal{X}=\wedge^{1} \mathcal{X}$ then, by (34), $\left.h\right\lrcorner$ is an antiderivation of degree 1 on the graded algebra $\wedge^{*} \mathcal{X}$, i.e., for all $n \in \mathbb{N}_{0}, \xi \in \wedge^{* n} \mathcal{X}$ and $\zeta \in \wedge^{*} \mathcal{X}$,

$$
\left.h\lrcorner(\xi \wedge \zeta)=(h\lrcorner \xi) \wedge \zeta+(-1)^{n} \xi \wedge(h\lrcorner \zeta\right) .
$$

Similar to the interior product, for any $\xi \in \wedge^{*} \mathcal{X}$, the exterior product induces a linear map

$$
\xi \wedge: \zeta \mapsto \xi \wedge \zeta
$$

from $\wedge^{*} \mathcal{X}$ to itself. Then, for all $\xi, \zeta \in \mathcal{X}^{*}=\wedge^{* 1} \mathcal{X}$ and $h, w \in \mathcal{X}$,

$$
(\xi \wedge)(\zeta \wedge)=-(\zeta \wedge)(\xi \wedge), \quad h\lrcorner w\lrcorner=-w\lrcorner h\lrcorner, \quad h\lrcorner(\xi \wedge)+(\xi \wedge) h\lrcorner=\xi(h) \mathbf{1}_{\wedge^{*} \mathcal{X}},
$$

because of (34). These equalities refer to the canonical anticommutation relations (CAR). Compare with (11) and (113).

(iv): Assume, from now on, that $\mathcal{X}$ is a finite-dimensional (complex) Hilbert space. Then we denote by $\overline{\mathfrak{j} \text { the }}$ unique antilinear map $\wedge \mathcal{X} \rightarrow \wedge^{*} \mathcal{X}$ such that, for any $n \in \mathbb{N}, z \in \wedge^{0} \mathcal{X} \doteq \mathbb{C}$ and $x_{1}, \ldots, x_{n} \in \mathcal{X}$,

$$
\mathfrak{j}\left(x_{1} \wedge \cdots \wedge x_{n}\right)=x_{1}^{*} \wedge \cdots \wedge x_{n}^{*} \quad \text { and } \quad \mathfrak{j}(z)=\bar{z} \in \wedge^{* 0} \mathcal{X} .
$$

Similarly, $\mathfrak{j}^{*}$ is the unique antilinear map $\wedge^{*} \mathcal{X} \rightarrow \wedge \mathcal{X}$ such that, for any $n \in \mathbb{N}, z \in \wedge^{* 0} \mathcal{X}$ and $x_{1}, \ldots, x_{n} \in \mathcal{X}$

$$
\mathfrak{j}^{*}\left(x_{1}^{*} \wedge \cdots \wedge x_{n}^{*}\right)=x_{1} \wedge \cdots \wedge x_{n} \quad \text { and } \quad \mathfrak{j}^{*}(z)=\bar{z} \in \wedge^{0} \mathcal{X} .
$$


Because of the Fréchet-Riesz representation theorem, $\mathfrak{j}$ and $\mathfrak{j}^{*}$ are bijective maps satisfying

$$
\mathfrak{j} \circ \mathfrak{j}^{*}=\mathbf{1}_{\wedge * \mathcal{X}} \quad \text { and } \quad \mathfrak{j}^{*} \circ \mathfrak{j}=\mathbf{1}_{\wedge \mathcal{X}} .
$$

The condition

$$
(\zeta, \xi) \doteq\left\langle\xi, j^{*}(\zeta)\right\rangle, \quad \xi, \zeta \in \wedge^{*} \mathcal{X}
$$

defines a scalar product $(\cdot, \cdot)$ in $\wedge^{*} \mathcal{X}$.

$\Lambda^{*} \mathcal{X}$ equipped with this scalar product is a Hilbert space $\mathcal{F}_{\mathcal{X}^{*}}$, the so-called fermionic Fock space associated with the (one-particle) Hilbert space $\mathcal{X}^{*}$. Moreover, for $\xi \in \mathcal{X}^{*}$ and $h \in \mathcal{X}$, the linear operators $h\lrcorner \in \mathcal{B}\left(\mathcal{F}_{\mathcal{X}^{*}}\right)$ and $\xi \wedge \in \mathcal{B}\left(\mathcal{F}_{\mathcal{X}^{*}}\right)$, respectively defined by (123) and (125), are related to each other by taking the adjoint:

$$
\left.\left.(\xi \wedge)^{*}=\mathfrak{j}^{*}(\xi)\right\lrcorner \quad \text { and } \quad(h\lrcorner\right)^{*}=\mathfrak{j}(h) \wedge .
$$

Their operator norms are respectively equal to

$$
\| h\lrcorner\left\|_{\mathcal{B}\left(\mathcal{F}_{\mathcal{X}^{*}}\right)}=\right\| h \|_{\mathcal{X}} \quad \text { and } \quad\|\xi \wedge\|_{\mathcal{B}_{\left(\mathcal{F}_{\mathcal{X}^{*}}\right)}}=\|\xi\|_{\mathcal{X}^{*}}
$$

by the CAR (126).

(v): Again because of (126), if $\mathcal{X}=\mathfrak{h}^{*}$, then there is an obvious $*$-isomorphism $\mathfrak{r}$ from $\mathrm{CAR}(\mathfrak{h})$ to $\overline{\mathcal{B}\left(\mathcal{F}_{\mathfrak{h}}\right)}$ that is uniquely defined by

$$
\left.\mathfrak{r}(a(\varphi))=\varphi^{*}\right\lrcorner, \quad \varphi \in \mathfrak{h} .
$$

The norm on $\operatorname{CAR}(\mathfrak{h})$ corresponds to the usual operator norm on $\mathcal{B}\left(\mathcal{F}_{\mathfrak{h}}\right)$ and, since the antisymmetric Fock space $\mathcal{F}_{\mathfrak{h}}$ has dimension equal to $2^{\text {dimh }}$, the CAR algebra is also finite-dimensional:

$$
\operatorname{dim}(\operatorname{CAR}(\mathfrak{h}))=2^{2 \operatorname{dim} \mathfrak{h}} .
$$

\section{Remark 6.4 (Tracial state)}

For any $*$-isomorphism $\mathfrak{r}$ from $\mathrm{CAR}(\mathfrak{h})$ to $\mathcal{B}\left(\mathcal{F}_{\mathfrak{h}}\right)$, the tracial state is written as a normalized trace $\operatorname{Tr}_{\mathcal{F}_{\mathfrak{h}}}$ on linear operators acting on the finite-dimensional fermionic Fock space $\mathcal{F}_{\mathfrak{h}}$ :

$$
\operatorname{tr}(A)=2^{-\operatorname{dimh}} \operatorname{Tr}_{\mathcal{F}_{\mathfrak{h}}}(\mathfrak{r}(A)), \quad A \in \mathrm{CAR}(\mathfrak{h}) .
$$

\section{Quantum Large Deviations}

\subsection{Large Deviations (LD) in Quantum Statistical Mechanics}

In probability theory, the law of large numbers refers to the convergence (at least in probability), as $n \rightarrow \infty$, of the average or empirical mean of $n$ independent identically distributed (i.i.d.) random variables towards their expected value (assuming it exists). The central limit theorem states that, as $n \rightarrow \infty$, the probability distribution of fluctuations (at scale $\sqrt{n}$ ) of this finite average around the expected value is a normal one (assuming the finiteness of the variance). The large deviation (LD) formalism quantitatively describes, for large $n \gg 1$, the probability of finding an empirical mean that differs from the expected value. The latter refers to rare events, by the law of large numbers, and an LD principle (LDP) gives their probability as exponentially small in the limit $n \rightarrow \infty$.

The concept of LD goes beyond the law of large numbers and complements the central limit theorem. Note, however, that, by the Bryc theorem [Bry93, Proposition 1], under some assumptions, the central limit theorem can result from an LDP. Moreover, the LD formalism does not necessarily require i.i.d. random variables, i.e., it can be studied for random variables that differ from empirical 
means of $n$ i.i.d. random variables. For a comprehensive account of the LD method, see [DSt89, DZ98].

The LD formalism is applied in physics since probability theory is the natural mathematical framework of (classical) statistical mechanics. See [E85, Le86, T09]. When an LDP holds true, there is a well-defined exponential rate of convergence of local quantities towards a thermodynamic one. In physics, this rate of convergence is frequently related to some notion of entropy. Moreover, in this case, a powerful extension of Laplace's method for infinite-dimensional spaces, well-known as Varadhan's lemma [V66], becomes available. See [DSt89, DZ98], in particular [DSt89, Theorem 2.1.10] or [DZ98, Theorem 4.3.1].

Since the eighties, the LD formalism has been successfully used in quantum statistical mechanics, initially at a time when the method of LD was not well-known in theoretical physics [VLP88, p. 63]. This was further developed by the "Dublin school" in 1988-1993. In the series of papers [VLP88, DLP89, VDLP90a, VDLP90b, DLP93] the authors studied boson systems in the continuum with interactions that only depend on occupation-number operators. This allowed them to map a thermodynamic problem in the bosonic Fock space to a classical probability problem for occupation numbers seen as a random variable. It gives a nice application of LDP to the occupation measure in order to solve a class of diagonal boson models, see [ZB01, Section 4.1.1]. In [LZ88] an LDP was proved for the distribution of the particle density (the so-called Kac distribution) in the Perfect and Mean-Field Bose gases.

More recently, non-diagonal boson models were fully studied using LDP, see, e.g., [BZ98, Br04, BZ08]. For instance, [BZ08] proves an LDP for both the (generalized) Kac distribution and the Bose condensate density in order to study the first order phase transition appearing in the thermodynamics of the superstable Weakly Imperfect Bose Gas (or Superstable Bogoliubov model). The phenomenon of Bose condensation in quantum statistical mechanics has inspired new LD studies in probability theory. See [ABK06a, ABK06b, A08, AK08, ACK11]. In these works, the random variables are quite elaborated mathematical objects within infinite-dimensional spaces. For instance, the authors in [ACK11] derive a variational formula for the limiting free energy of a general Bose gas with pair interactions on the continuum by using marked Poisson point processes and an LD analysis of the so-called stationary empirical field.

The use of the LD formalism in quantum statistical mechanics is not restricted to Bose systems. In 1987-1989, this method was used to analyze quantum spin systems [DP87, CLR88, RW89]. These analyses included certain types of translation-invariant fermionic models with long-range components on the lattice (e.g., reduced BCS models) via the quantum spin representation of fermions. Much more recently, as already mentioned, [LLS00, GLM02, Br04] focused on LD for the particle density in a sub-domain both for the perfect and for rarefied quantum gases (Fermi, Bose or Boltzmann statistics). Finally, in recent years the LD formalism was used to study quantum lattice systems at thermal equilibrium, as summarized in the works [LLS00, GLM02, NR04, LR05, O10, OR11, dRMNS15], to name a few examples. In most cases, equilibrium states are, by definition, infinitevolume KMS states, which are supposed to be unique (i.e., there is no phase transition).

Note that our list of results related to LD methods in quantum statistical mechanics is not exhaustive. Our aim is to show its usefulness in solving important models of quantum statistical physics. We also observe at this point that LD methods do not form a real "theory", as explained in [DSt89, Preface], and they have been used in many different ways in thermodynamic studies of quantum (many-body) models. For instance, in [VLP88], etc., LD methods are used by mapping the manybody problem to a relatively simple probabilistic one, for very specific models. In [ACK11], the partition function for a general interacting boson model in the canonical ensemble is reformulated via the Feynman-Kac formula in terms of interacting Brownian bridges in order to later apply LD methods to some stochastic process.

In the recent works [LLS00, GLM02, NR04, LR05, HMO07, O10, OR11, dRMNS15] on quantum 
lattice systems, the LD method is applied to (KMS) states on quantum spin or CAR $C^{*}$-algebra, via the algebraic formulation of Quantum Mechanics. See, e.g., [BdSP17a, Section 2]. This approach is also used in the present work to study LD properties associated with any observable for weakly interacting fermionic gases at thermal equilibrium. We thus briefly present this approach in Section 7.2 .

\subsection{LD Formalism for Quantum Observables}

Let $\mathcal{X}$ be a unital $C^{*}$-algebra. In the algebraic formulation of Quantum Mechanics, each self-adjoint element $A^{*}=A$ of $\mathcal{X}$, also called an observable, represents some apparatus (or measuring device), and its spectrum, denoted by $\operatorname{spec}(A)$, corresponds to all values that can arise by measuring the corresponding physical quantity. The state of the physical system is represented by states on the $C^{*}$ algebra $\mathcal{X}$, that is, by definition, continuous linear functionals $\rho$ which are normalized and positive, i.e., $\rho(\mathbf{1})=1$ and $\rho\left(A^{*} A\right) \geq 0$ for all $A \in \mathcal{X}$.

The commutative $C^{*}$-subalgebra of $\mathcal{X}$ generated by any self-adjoint element $A^{*}=A \in \mathcal{X}$ is isomorphic to the algebra of continuous functions on the compact $\operatorname{set} \operatorname{spec}(A) \subset \mathbb{R}$. Hence, by the Riesz-Markov theorem (or Riesz representation theorem), for any observable $A \in \mathcal{X}$ and state $\rho \in \mathcal{X}^{*}$, there is a unique probability measure $\mu_{\rho, A}$ on $\mathbb{R}$ such that

$$
\mu_{\rho, A}(\operatorname{spec}(A))=1 \quad \text { and } \quad \rho(f(A))=\int_{\mathbb{R}} f(x) \mu_{\rho, A}(\mathrm{~d} x)
$$

for all complex-valued continuous functions $f \in C(\mathbb{R} ; \mathbb{C})$. Note that, for any observable $A \in \mathcal{X}$, $y \in \mathbb{R}$ and any Borel subset $\mathcal{O} \subset \mathbb{R}$,

$$
\mu_{\rho, A-y 1}(\mathcal{O})=\mu_{\rho, A}(\mathcal{O}+y),
$$

by the uniqueness of the probability measure in the Riesz-Markov theorem. $\mu_{\rho, A}$ is called the distribution of the observable $A$ in the state $\rho$.

Since distributions are, by construction, probability measures on the complete, separable space $\mathbb{R}$, the LD formalism naturally arises: A rate function is a lower semi-continuous function $\mathrm{I}: \mathbb{R} \rightarrow$ $[0, \infty]$. If $\mathrm{I}$ is not the $\infty$-constant function and has compact level sets, i.e., if $\mathrm{I}^{-1}([0, m])=\{x \in$ $\mathbb{R}: \mathrm{I}(x) \leq m\}$ is compact for any $m \geq 0$, then one says that $\mathrm{I}$ is a good rate function. A sequence $\left\{A_{l}\right\}_{l \in \mathbb{R}^{+}} \subset \mathcal{X}$ of observables satisfies the LD upper bound in a state $\rho \in \mathcal{X}^{*}$ with speed $\mathfrak{n}_{l} \in \mathbb{R}$, $l \in \mathbb{R}^{+}$, and rate function I if, for any closed subset $\mathcal{C}$ of $\mathbb{R}$,

$$
\limsup _{l \rightarrow \infty} \frac{1}{\mathfrak{n}_{l}} \ln \mu_{\rho, A_{l}}(\mathcal{C}) \leq-\inf _{\mathcal{C}} \mathrm{I}(x)
$$

and it satisfies the LD lower bound in (a state $\rho$ and) the closed interval $\mathcal{I} \subseteq \mathbb{R}$ if, for any open subset $\mathcal{G} \subset \mathcal{I}$ of $\mathbb{R}$

$$
\liminf _{l \rightarrow \infty} \frac{1}{\mathfrak{n}_{l}} \ln \mu_{\rho, A_{l}}(\mathcal{G}) \geq-\inf _{\mathcal{G}} \mathrm{I}(x) .
$$

If both upper and lower bound are satisfied, we say that $\left\{A_{l}\right\}_{l \in \mathbb{R}^{+}} \subset \mathcal{X}$ satisfies a large deviation principle (LDP) in a state $\rho$ and the closed interval $\mathcal{I} \subseteq \mathbb{R}$. The LDP is called weak if the upper bound in (131) holds only for compact sets $\mathcal{C}$.

One of the most important consequences of an LDP satisfied by $\left\{A_{l}\right\}_{l \in \mathbb{R}^{+}} \subset \mathcal{X}$ in the state $\rho \in \mathcal{X}^{*}$ and in $\mathcal{I}=\mathbb{R}$ is Varadhan's lemma, which says, in this context, that the limiting generating function

$$
\mathrm{G}(f) \doteq \lim _{l \rightarrow \infty} \frac{1}{\mathfrak{n}_{l}} \ln \int_{\mathbb{R}} \exp \left(\mathfrak{n}_{l} f(x)\right) \mu_{\rho, A_{l}}(\mathrm{~d} x)=\lim _{l \rightarrow \infty} \frac{1}{\mathfrak{n}_{l}} \ln \rho\left(\mathrm{e}^{\mathfrak{n}_{l} f\left(A_{l}\right)}\right)
$$


is equal, for any bounded and continuous real-valued function $f \in C_{b}(\mathbb{R})$, to

$$
\mathrm{G}(f)=\sup _{x \in \mathbb{R}}\{f(x)-\mathrm{I}(x)\} .
$$

See [DSt89, Theorem 2.1.10] or [DZ98, Theorem 4.3.1].

The converse holds true when one can ensure that no loss of mass appears in the limit $l \rightarrow \infty$ : We say that a family $\left\{A_{l}\right\}_{l \in \mathbb{R}^{+}} \subset \mathcal{X}$ of observables is exponentially tight in the state $\rho \in \mathcal{X}^{*}$ if the sequence $\left\{\mu_{\rho, A_{l}}\right\}_{l \in \mathbb{R}^{+}}$is exponentially tight. This means that, for every $D<\infty$, there exists a compact set $\mathcal{C}_{D} \subset \mathbb{R}$ such that

$$
\limsup _{l \rightarrow \infty} \frac{1}{\mathfrak{n}_{l}} \ln \mu_{\rho, A_{l}}\left(\mathbb{R} \backslash \mathcal{C}_{D}\right)<-D
$$

Then, by [Bry90] (or [DZ98, Theorem 4.4.2]), the existence of $\mathrm{G}(f)$ for all $f \in C_{b}(\mathbb{R})$, together with the exponential tightness of $\left\{A_{l}\right\}_{l \in \mathbb{R}^{+}} \subset \mathcal{X}$ in the state $\rho \in \mathcal{X}^{*}$, implies that $\left\{A_{l}\right\}_{l \in \mathbb{R}^{+}}$satisfies an LDP with the good rate function I defined, for any $x \in \mathbb{R}$, by

$$
\mathrm{I}(x) \doteq \sup _{f \in C_{b}(\mathbb{R})}\{f(x)-\mathrm{G}(f)\} .
$$

To prove the existence of an LDP, the knowledge of $\mathrm{G}(f)$ for all $f \in C_{b}(\mathbb{R})$ is not necessary. Instead, by the Gärtner-Ellis theorem, one can consider the (limiting) logarithmic moment generating function. See, e.g., [DSt89, Section 2.2] or [DZ98, Section 2.3 or 4.5]. For any sequence $\left\{A_{l}\right\}_{l \in \mathbb{R}^{+}} \subset$ $\mathcal{X}$ of observables and state $\rho \in \mathcal{X}^{*}$, this function is defined by

$$
\mathrm{J}(s) \doteq \lim _{l \rightarrow \infty} \frac{1}{\mathfrak{n}_{l}} \ln \left(\int_{\mathbb{R}} \mathrm{e}^{s \mathfrak{n}_{l} x} \mu_{\rho, A_{l}}(\mathrm{~d} x)\right)=\lim _{l \rightarrow \infty} \frac{1}{\mathfrak{n}_{l}} \ln \rho\left(\mathrm{e}^{s^{\mathfrak{n}_{l}} A_{l}}\right), \quad s \in \mathbb{R} .
$$

It equals $\mathrm{G}\left(h_{s}\right)$ with $h_{s}(x) \doteq s x$ for $s, x \in \mathbb{R}$ and it is the standard limiting logarithmic moment generating function of the sequence $\left\{\mu_{\rho, A_{l}}\right\}_{l \in \mathbb{R}^{+}}$of measures on $\mathbb{R}$.

Then, a sufficient condition to ensure that a sequence of observables satisfies an LDP is given by the Gärtner-Ellis theorem (see, e.g., [DZ98, Corollary 4.5.27]), which is better adapted to our methods than Bryc's inverse Varadhan lemma [DZ98, Theorem 4.4.2]. In our context, this refers to the following assertion:

\section{Theorem 7.1 (Gärtner-Ellis)}

Let $\rho \in \mathcal{X}^{*}$ be a state. Take any sequence $\left\{A_{l}\right\}_{l \in \mathbb{R}^{+}} \subset \mathcal{X}$ of observables that is exponentially tight in the state $\rho$. Assume the existence in a closed interval $\mathcal{J} \subseteq \mathbb{R}$ of the limiting logarithmic moment generating function J defined by (133). Then, one has:

(LD1) $\left\{A_{l}\right\}_{l \in \mathbb{R}^{+}}$satisfies the LD upper bound (131) in the state $\rho$, with rate function I given as the Legendre-Fenchel transform of J, that is, the convex lower semi-continuous map from $\mathbb{R}$ to $(-\infty, \infty]$ defined, for any $x \in \mathbb{R}$, by

$$
\mathrm{I}(x) \doteq \sup _{s \in \mathcal{J}}\{s x-\mathrm{J}(s)\} .
$$

(LD2) If $\mathrm{J}$ is differentiable for all $s \in \mathcal{J}$ and

$$
\lim _{l \rightarrow \infty} \rho\left(A_{l}\right) \in\left(\inf \mathrm{J}^{\prime}(\mathcal{J}), \sup \mathrm{J}^{\prime}(\mathcal{J})\right),
$$

then $\left\{A_{l}\right\}_{l \in \mathbb{R}^{+}}$satisfies the LD lower bound (132) in (the state $\rho$ and) the closed interval

$$
\mathcal{I} \doteq\left[\inf J^{\prime}(\mathcal{J}), \sup J^{\prime}(\mathcal{J})\right]
$$

with good rate function I given by (134). 
Proof. (LD1) and (LD2) for $\mathcal{I}=\mathbb{R}$ are consequences of [DSt89, Theorem 2.2.4] and [DZ98, Corollary 4.5.27], respectively. The case $\mathcal{I} \varsubsetneqq \mathbb{R}$ follows straightforwardly from the previous one. For an explicit proof in the special case of finite intervals $\mathcal{I} \varsubsetneqq \mathbb{R}$, see [JOPP12, Appendix A.2].

[Bry93] explains how, in the zero mean case, the central limit theorem is deduced from the existence of the limiting logarithmic moment generating function on a neighborhood - in the complex plane - of the origin. Bryc's result [Bry93, Proposition 1] can be rewritten in our context as follows:

\section{Theorem 7.2 (Bryc)}

Take a sequence $\left\{A_{l}\right\}_{l \in \mathbb{R}^{+}} \subset \mathcal{X}$ of observables and fix a state $\rho \in \mathcal{X}^{*}$. If the limiting logarithmic moment generating function $\mathrm{J}$ defined by (133) exists and admits an analytic continuation to a neighborhood of $s=0 \in \mathbb{C}$, then the family $\mu_{\sqrt{\mathfrak{n}_{l}}\left(A_{l}-\rho\left(A_{l}\right)\right.}, l \in \mathbb{R}^{+}$, of measures converges in the weak ${ }^{*}$-topology to the normal (or Gaussian) distribution $\mathcal{N}_{0, \sigma^{2}}$ with variance $\sigma^{2}=\mathrm{J}^{\prime \prime}(0)$.

Note that [Bry93, Proposition 2] gives a condition to ensure the hypothesis of this proposition by using an LDP. See also [DSt89, Lemma 2.2.9].

\section{Application of LD to Quantum Statistical Mechanics}

\subsection{Spin or CAR Algebra}

The applications we have in mind refer to quantum spins or fermions on a $d$-dimensional lattice $\mathbb{Z}^{d}$, $d \in \mathbb{N}$. Denote by $\mathcal{P}_{f}\left(\mathbb{Z}^{d}\right) \subset 2^{\mathbb{Z}^{d}}$ the set of all finite subsets of $\mathbb{Z}^{d}$. Fix also a finite set $S$, the elements of which represent the pure spin states of a quantum particle in any arbitrary site of the lattice.

Then, in the algebraic formulation of Quantum Mechanics, it is standard to associate with the pair $\left(\mathbb{Z}^{d}, \mathrm{~S}\right)$ a unital $C^{*}$-algebra $\mathcal{X}=\mathcal{U}$ generated by a net $\left\{\mathcal{U}_{\Lambda}\right\}_{\Lambda \in \mathcal{P}_{f}\left(\mathbb{Z}^{d}\right)} \subset \mathcal{U}$ of finite-dimensional subalgebras. In fact, $\mathcal{U}$ is a so-called quasi-local algebra with respect to this generating net, see [BR96a, Definition 2.6.3.]. For quantum spin systems,

$$
\mathcal{U}_{\Lambda}=\mathcal{B}\left(\mathbb{C}^{|\Lambda| \times|S|}\right)
$$

while, for fermions on the lattice,

$$
\left.\mathcal{U}_{\Lambda}=\operatorname{CAR}\left(\ell^{2}(\Lambda \times \mathrm{S})\right) \equiv \mathcal{B}\left(\mathcal{F}_{\ell^{2}(\Lambda \times \mathrm{S})}\right)\right) \equiv \mathcal{B}\left(\mathbb{C}^{2^{2|\Lambda| \times|\mathrm{S}|}}\right)
$$

is the (local or finite-volume) CAR $\left(C^{*}\right)$ algebra associated with the one-particle Hilbert space $\ell^{2}(\Lambda \times \mathrm{S})$, as explained in Section 6.1. Recall that $\mathcal{B}(\mathfrak{h})$ denotes the $C^{*}$-algebra of bounded linear operators acting on $\mathfrak{h}$ (Notation 1.3), while $\mathcal{F}_{\mathfrak{h}}$ is the fermionic Fock space associated with the one-particle Hilbert space $\mathfrak{h}$ (Section 6.4). (In the bosonic case, a similar formalism can be developed with the use of CCR (Canonical Commutation Relations) algebras. See [BR96b, EK98].)

In the context of self-dual CAR algebra, described in Section 6.2, for any $\Lambda \in \mathcal{P}_{f}\left(\mathbb{Z}^{d}\right), \mathcal{U}_{\Lambda}$ can be seen as the self-dual CAR algebra associated with $\ell^{2}(\Lambda \times S) \oplus \ell^{2}(\Lambda \times S)^{*}$. Then, the CAR algebra $\mathcal{U}$ generated by the net $\left\{\mathcal{U}_{\Lambda}\right\}_{\Lambda \in \mathcal{P}_{f}\left(\mathbb{Z}^{d}\right)}$ is, in turn, the self-dual CAR algebra associated with $\ell^{2}\left(\mathbb{Z}^{d} \times \mathrm{S}\right) \oplus \ell^{2}\left(\mathbb{Z}^{d} \times \mathrm{S}\right)^{*}$, similar to the finite-dimensional case. Note that

$$
\ell^{2}(\Lambda \times \mathrm{S}) \oplus \ell^{2}(\Lambda \times \mathrm{S})^{*} \equiv \ell^{2}\left(\Lambda ; \mathcal{H}_{\mathrm{S}}\right), \quad \Lambda \subset \mathbb{Z}^{d}
$$

Compare with Section 5.3. 


\subsection{Thermodynamic Sequences of Observables}

The speed of the LD we study is typically $\mathfrak{n}_{l}=\left|\Lambda_{l}\right|, l \in \mathbb{R}^{+}$, where $\left\{\Lambda_{l}\right\}_{l \in \mathbb{R}^{+}}$is the sequence of cubic boxes defined by (99). This choice to define the thermodynamic limit is technically convenient, but not essential. The arguments can be adapted to more general van Hove nets of boxes. A typical sequence $\left\{A_{l}\right\}_{l \in \mathbb{R}^{+}}$of observables analyzed in the context of Section 7.2 is the sequence of space averages, of some fixed observable $A$, over the boxes $\Lambda_{l}$.

Denote by $\left\{\chi_{x}\right\}_{x \in \mathbb{Z}^{d}}$ the family of $*$-automorphisms of the $C^{*}$-algebra $\mathcal{U}$ that implements the action of the group of lattice translations. When $\mathcal{U}=\operatorname{CAR}\left(\ell^{2}\left(\mathbb{Z}^{d} \times \mathrm{S}\right)\right)$, this family is uniquely defined by the conditions

$$
\chi_{x}(a(\varphi))=a(\varphi(x+\cdot, \cdot)), \quad \varphi \in \ell^{2}\left(\mathbb{Z}^{d} \times \mathrm{S} ; \mathbb{C}\right) .
$$

The definition of this family is also straightforward for quantum spin systems and we omit the details.

Then, the empirical mean $\left\{\mathrm{M}_{l}^{A}\right\}_{l \in \mathbb{R}^{+}}$of observables is defined, for any $l \in \mathbb{R}^{+}$and $A \in \mathcal{U}$, by

$$
\mathrm{M}_{l}^{A} \doteq \frac{1}{\left|\Lambda_{l}\right|} \sum_{x \in \Lambda_{l}} \chi_{x}(A)
$$

This case obviously includes the sequence $\left\{D_{l}\right\}_{l \in \mathbb{R}^{+}} \subset \mathcal{X}$ of fermionic density observables, which is defined, for any $l \in \mathbb{R}^{+}$, by

$$
\mathrm{D}_{l} \doteq \frac{1}{\left|\Lambda_{l}\right|} \sum_{x \in \Lambda_{l}, \mathrm{~s} \in \mathrm{S}} a^{*}\left(\mathfrak{e}_{x, \mathrm{~s}}\right) a\left(\mathfrak{e}_{x, \mathrm{~s}}\right) \in \mathcal{U}_{\Lambda_{l}} \cap \mathcal{U}^{+} \subset \mathcal{U}
$$

Here, $\left\{\mathfrak{e}_{x, \mathrm{~s}}\right\}_{(x, \mathrm{~s}) \in \mathbb{Z}^{d} \times \mathrm{S}}$ is the canonical orthonormal basis of $\ell^{2}\left(\mathbb{Z}^{d} \times \mathrm{S}\right)$, defined, like (101), by

$$
\mathfrak{e}_{x, \mathrm{~s}}(y, \mathrm{t}) \doteq \delta_{x, y} \delta_{\mathrm{s}, \mathrm{t}}, \quad x, y \in \mathbb{Z}^{d}, \quad \mathrm{~s}, \mathrm{t} \in \mathrm{S}
$$

Observe also that, for any $A \in \mathcal{U},\left\{\mathrm{M}_{l}^{A}\right\}_{l \in \mathbb{R}^{+}} \subset \mathcal{X}$ is exponentially tight in any state $\rho \in \mathcal{U}^{*}$ since

$$
\operatorname{spec}\left(\mathrm{M}_{l}^{A}\right) \subset\left[-\|A\|_{\mathcal{U}},\|A\|_{\mathcal{U}}\right], \quad l \in \mathbb{R}^{+} .
$$

See Equation (130).

A similar sequence of observables can be defined via a so-called interaction, a standard notion in the context of quantum spin and lattice Fermi systems. This refers to a family $\Psi=\left\{\Psi_{\Lambda}\right\}_{\Lambda \in \mathcal{P}_{f}\left(\mathbb{Z}^{d}\right)} \subset$ $\mathcal{U}^{+}$such that $\Psi_{\Lambda}=\Psi_{\Lambda}^{*} \in \mathcal{U}_{\Lambda} \cap \mathcal{U}^{+}$for all $\Lambda \in \mathcal{P}_{f}\left(\mathbb{Z}^{d}\right)$ and $\Psi_{\emptyset}=0$. Here, for quantum spin systems, $\mathcal{U}^{+}=\mathcal{U}$, while in fermion systems on the lattice, $\mathcal{U}^{+} \varsubsetneqq \mathcal{U}$ is the even subalgebra of $\mathcal{U}=\operatorname{CAR}\left(\ell^{2}\left(\mathbb{Z}^{d} \times \mathrm{S}\right)\right)$. See end of Section 6.1. Then, a thermodynamic sequence of observables that can also be studied is defined, for any interaction $\Psi$ and $l \in \mathbb{R}^{+}$, by

$$
\mathrm{E}_{l}^{\Psi} \doteq \frac{1}{\left|\Lambda_{l}\right|} \sum_{\Lambda \subseteq \Lambda_{l}} \Psi_{\Lambda} \in \mathcal{U}_{\Lambda_{l}} \cap \mathcal{U}^{+} \subset \mathcal{U}
$$

It can be seen as a finite-volume, energy-density observable of the interaction $\Psi$, or the energy-perunit-volume observable associated with the interaction $\Psi$ and the box $\Lambda_{l}$.

An important class of interactions are the so-called translation-invariant, finite-range interactions:

- A translation-invariant interaction is an interaction $\Psi$ satisfying

$$
\Psi_{x+\Lambda}=\chi_{x}\left(\Psi_{\Lambda}\right), \quad \Lambda \in \mathcal{P}_{f}\left(\mathbb{Z}^{d}\right), x \in \mathbb{Z}^{d}
$$


- An interaction $\Psi$ has finite-range iff there is some $R<\infty$ such that $\varnothing(\Lambda)>R$ implies $\Psi_{\Lambda}=0$, where, for any $\Lambda \in \mathcal{P}_{f}\left(\mathbb{Z}^{d}\right)$,

$$
\phi(\Lambda) \doteq \max _{\left(x_{1}, \ldots, x_{d}\right),\left(y_{1}, \ldots, y_{d}\right) \in \Lambda} \sqrt{\left|x_{1}-y_{1}\right|^{2}+\cdots+\left|x_{d}-y_{d}\right|^{2}}
$$

For instance, let $\Lambda_{0} \in \mathcal{P}_{f}\left(\mathbb{Z}^{d}\right), A=A^{*} \in \mathcal{U}_{\Lambda_{0}} \cap \mathcal{U}^{+}$, and $\Psi^{A}$ be the interaction defined by $\Psi_{\Lambda}^{A} \doteq \chi_{x}(A)$ for $\Lambda=\Lambda_{0}+x$ with $x \in \mathbb{Z}^{d}$ and $\Psi_{\Lambda}^{A}=0$ otherwise. This is an example of translationinvariant and finite-range interactions. Additionally, note that

$$
\mathrm{E}_{l}^{\Psi^{A}}=\mathrm{M}_{l}^{A}+\mathcal{O}\left(l^{-1}\right)
$$

in the $C^{*}$-algebra $\mathcal{U}$. The finite-volume energy-density observables can be approximated by empirical means even for more general interactions: If $\Psi$ is translation-invariant and finite-range, then straightforward computations (see as an example [BdSP13, Definition 1.24 and Lemma 4.17]) show that

$$
\lim _{l \rightarrow \infty}\left\|\mathrm{E}_{l}^{\Psi}-\mathrm{M}_{l}^{\mathfrak{E}^{\Psi}}\right\|_{\mathcal{U}}=0
$$

where $\mathrm{M}_{l}^{\mathfrak{E}^{\Psi}}$ is the empirical mean (138) associated with the energy-density observable

$$
\mathfrak{E}^{\Psi} \doteq \sum_{\Lambda \in \mathcal{P}_{f}(\mathfrak{L}), \Lambda \ni 0} \frac{\Psi_{\Lambda}}{|\Lambda|} \in \mathcal{U}^{+} .
$$

Moreover, in this case, $\left\{\mathrm{E}_{l}^{\Psi}\right\}_{l \in \mathbb{R}^{+}} \subset \mathcal{X}$ is exponentially tight in any state $\rho \in \mathcal{U}^{*}$. Equation (143), however, does not have to hold when the interaction $\Psi$ is not translation-invariant or finite-range.

\subsection{Exponentially Ergodic States}

Ergodic states are states whose space averages or empirical means of observables exist and do not fluctuate, in the limit of large volumes:

\section{Definition 8.1 (Ergodic states)}

We say that a state ${ }^{15} \rho \in \mathcal{U}^{*}$ is ergodic if, for all $A \in \mathcal{U}, \rho\left(\mathrm{M}_{l}^{A}\right)$ converges, as $l \rightarrow \infty$, and

$$
\lim _{l \rightarrow \infty} \rho\left(\left(\mathrm{M}_{l}^{A}-\rho\left(\mathrm{M}_{l}^{A}\right) \mathbf{1}\right)^{*}\left(\mathrm{M}_{l}^{A}-\rho\left(\mathrm{M}_{l}^{A}\right) \mathrm{I}\right)\right)=0 .
$$

For any $M=M^{*} \in \mathcal{U}$, the non-negative number

$$
\rho\left((M-\rho(M) 1)^{2}\right) \geq 0
$$

quantifies the fluctuation, around the expected value $\rho(M)$, of the physical quantity associated with the observable $M$ in the state $\rho$. It is the variance of any real-valued, random variable with distribution given by $\mu_{\rho, M}$, as defined by (130).

Because of (142) and (143), the ergodicity of states can be expressed equivalently in terms of the sequences $\left\{\mathrm{E}_{l}^{\Psi}\right\}_{l \in \mathbb{R}^{+}}(141)$ :

\section{Lemma 8.2 (Ergodic states)}

A state $\rho \in \mathcal{U}^{*}$ is ergodic iff, for any translation-invariant and finite-range interaction $\Psi, \rho\left(\mathrm{E}_{l}^{\Psi}\right)$ converges, as $l \rightarrow \infty$, and

\footnotetext{
${ }^{15}$ I.e., $\rho(\mathbf{1})=1$ and $\rho\left(A^{*} A\right) \geq 0$ for $A \in \mathcal{U}$.
}

$$
\lim _{l \rightarrow \infty} \rho\left(\left(\mathrm{E}_{l}^{\Psi}-\rho\left(\mathrm{E}_{l}^{\Psi}\right) \mathbf{\perp}\right)^{2}\right)=0 .
$$


Proof. By the Cauchy-Schwarz inequality for states [BR96a, Lemma 2.3.10 (b)], Definition 8.1 can be restricted to observables $A \in \mathcal{U}$ : A state $\rho$ is ergodic iff $\rho\left(\mathrm{M}_{l}^{A}\right)$ converges, as $l \rightarrow \infty$, and

$$
\lim _{l \rightarrow \infty} \rho\left(\left(\mathrm{M}_{l}^{A}-\rho\left(\mathrm{M}_{l}^{A}\right) \mathrm{I}\right)^{2}\right)=0, \quad A=A^{*} \in \mathcal{U}
$$

By (143) and the density of the set of finite-volume elements of $\mathcal{U}$, the assertion then follows.

The existence of such ergodic states is well-known. For instance, in the case of translationinvariant states, ergodicity is equivalent to extremality (see, e.g., [BdSP13, Section 1.2 and Chapter 4]): Denote the set of all translation-invariant states by

$$
E_{1} \doteq \bigcap_{x \in \mathbb{Z}^{d}, A \in \mathcal{U}}\left\{\rho \in \mathcal{U}^{*}: \rho(\mathbf{1})=1, \rho\left(A^{*} A\right) \geq 0 \quad \text { with } \rho=\rho \circ \chi_{x}\right\}
$$

Ergo, for any state $\rho \in E_{1}$,

$$
\rho\left(\mathrm{M}_{l}^{A}\right)=\rho(A), \quad A \in \mathcal{U},
$$

where $\mathrm{M}_{l}^{A} \in \mathcal{U}$ is the empirical mean defined by (138). For translation-invariant states, one can view $\mathrm{M}_{l}^{A}$ as the empirical mean of $\left|\Lambda_{l}\right|$ (not necessarily independent) identically distributed (i.d.) observables for any $l \in \mathbb{R}^{+}$. By [BdSP13, Remark 1.4, Theorem 1.16, Corollary 4.6], the set of ergodic states is the set $\mathcal{E}_{1}$ of extreme points of the weak* ${ }^{*}$-compact convex set $E_{1}$. Moreover, by [BdSP13, Remark 1.4, Corollary 4.6], $\mathcal{E}_{1}$ is a weak*-dense $G_{\delta}$ subset of $E_{1}$. (Up to an affine homeomorphism, $E_{1}$ is the Poulsen simplex.)

Definition 8.1 (or Lemma 8.2) does not automatically yield an LDP for sequences of finite-volume energy-density observables. We thus define the following subset of ergodic states:

\section{Definition 8.3 (Exponentially ergodic states)}

Exponentially ergodic states are ergodic states $\rho$ with the property that, for any translation-invariant and finite-range interaction $\Psi$, the sequence $\left\{\mathrm{E}_{l}^{\Psi}\right\}_{l \in \mathbb{R}^{+}}$(141) satisfies an LDP in some closed interval $\mathcal{I} \ni \lim _{l \rightarrow \infty} \rho\left(\mathrm{E}_{l}^{\Psi}\right)$ with a good rate function $\mathrm{I}$.

Using results of this paper, we will prove in $[\mathrm{BdSPX}]$ that translation invariant (or more generally periodic) KMS states of weakly interacting fermions on the lattice are exponentially ergodic states.

\subsection{Thermal Equilibrium States for Fermion Systems}

By definition, an interaction is a family $\Phi=\left\{\Phi_{\Lambda}\right\}_{\Lambda \in \mathcal{P}_{f}(\mathfrak{L})}$ of (even) elements such that $\Phi_{\Lambda}=\Phi_{\Lambda}^{*} \in$ $\mathcal{U}_{\Lambda} \cap \mathcal{U}^{+}$for all $\Lambda \in \mathcal{P}_{f}\left(\mathbb{Z}^{d}\right)$ and $\Phi_{\emptyset}=0$. For quantum spin systems, $\mathcal{U}^{+}=\mathcal{U}$, while in fermion systems on the lattice, $\mathcal{U}^{+} \varsubsetneqq \mathcal{U}$ is the even subalgebra.

Fix two interactions $\Phi_{\mathrm{f}}$ and $\Phi_{\mathrm{i}}$ respectively representing the free (or unperturbed) and interparticle components of the total interaction $\Phi=\Phi_{\mathrm{f}}+\Phi_{\mathrm{i}}$. These interactions define the quantum spin or fermion system via a sequence of (internal) energy observables or Hamiltonians

$$
H_{L_{\mathrm{f}}, L_{\mathrm{i}}} \doteq \sum_{\Lambda_{1} \subseteq \Lambda_{L_{\mathrm{f}}}} \Phi_{\mathrm{f}, \Lambda_{1}}+\sum_{\Lambda_{2} \subseteq \Lambda_{L_{\mathrm{i}}}} \Phi_{\mathrm{i}, \Lambda_{2}}=\left|\Lambda_{L_{\mathrm{f}}}\right| \mathrm{E}_{L_{\mathrm{f}}}^{\Phi_{\mathrm{f}}}+\left|\Lambda_{L_{\mathrm{i}}}\right| \mathrm{E}_{L_{\mathrm{i}}}^{\Phi_{\mathrm{i}}}, \quad L_{\mathrm{f}}, L_{\mathrm{i}} \in \mathbb{R}_{0}^{+}
$$

Then, the Gibbs state ${ }^{16} \rho_{L_{\mathrm{f}}, L_{\mathrm{i}}} \in \mathcal{U}_{\Lambda_{L_{\mathrm{f}}}}^{*}$ is defined, for any $L_{\mathrm{f}}, L_{\mathrm{i}} \in \mathbb{R}_{0}^{+}$and $\beta \in \mathbb{R}^{+}$, by

$$
\rho_{L_{\mathrm{f}}, L_{\mathrm{i}}}(A) \doteq \frac{\operatorname{tr}\left(A \mathrm{e}^{-\beta H_{L_{\mathrm{f}}, L_{\mathrm{i}}}}\right)}{\operatorname{tr}\left(\mathrm{e}^{-\beta H_{L_{\mathrm{f}}, L_{\mathrm{i}}}}\right)}, \quad A \in \mathcal{U} .
$$

\footnotetext{
${ }^{16}$ Or finite-volume thermal-equilibrium state, that is, the unique state minimizing the finite-volume free energy.
} 
Here, $\operatorname{tr} \in \mathcal{U}^{*}$ is the tracial state of the $C^{*}$-algebra $\mathcal{U}$. Cf. Lemma 2.10. For fermion systems, see Definition 2.9 and Remark 6.4. For any $L_{\mathrm{f}}, L_{\mathrm{i}} \in \mathbb{R}_{0}^{+}, \rho_{L_{\mathrm{f}}, L_{\mathrm{i}}}$ is the unique KMS state [BR96b, Section 5.3] associated with the inverse temperature $\beta \in \mathbb{R}^{+}$and the continuous group $\left\{\tau_{t}^{\left(L_{\mathrm{f}}, L_{\mathrm{i}}\right)}\right\}_{t \in \mathbb{R}}$ of $*$-automorphisms of $\mathcal{U}$ defined by

$$
\tau_{t}^{\left(L_{\mathrm{f}}, L_{\mathrm{i}}\right)}(A)=\mathrm{e}^{i t H_{L_{\mathrm{f}}, L_{\mathrm{i}}}} A \mathrm{e}^{-i t H_{L_{\mathrm{f}}, L_{\mathrm{i}}}}, \quad A \in \mathcal{U}
$$

The limit $\left(L_{\mathrm{f}}, L_{\mathrm{i}}\right) \rightarrow(\infty, \infty)$ refers to the thermodynamic limit. Similar to the set of probability measures in the commutative setting, the set of all states on any $C^{*}$-algebra is a weak*-compact (convex) set and so, for any interaction $\Phi_{\mathrm{f}}, \Phi_{\mathrm{i}}$, the family $\left\{\rho_{L_{\mathrm{f}}, L_{\mathrm{i}}}\right\}_{L_{\mathrm{f}}, L_{\mathrm{i}} \in \mathbb{R}_{0}^{+}}$has at least one weak*accumulation point, which is a state. If $\Phi_{\mathrm{f}}, \Phi_{\mathrm{i}}$ are finite-range interactions (Section 8.2), the groups $\left\{\tau_{t}^{\left(L_{\mathrm{f}}, L_{\mathrm{i}}\right)}\right\}_{t \in \mathbb{R}}, L_{\mathrm{f}}, L_{\mathrm{i}} \in \mathbb{R}_{0}^{+}$, converge strongly, as $\left(L_{\mathrm{f}}, L_{\mathrm{i}}\right) \rightarrow(\infty, \infty)$, to a strongly continuous group $\left\{\tau_{t}\right\}_{t \in \mathbb{R}}$ of $*$-automorphisms on $\mathcal{U}$. See for instance [BdSP17a, Theorem 4.8]. By [BR96b, Proposition 5.3.25], in this case, any weak ${ }^{*}$-accumulation point $\rho$ of $\left\{\rho_{L_{\mathrm{f}}, L_{\mathrm{i}}}\right\}_{L_{\mathrm{f}}, L_{\mathrm{i}} \in \mathbb{R}_{0}^{+}}$is a KMS state associated with $\left\{\tau_{t}\right\}_{t \in \mathbb{R}}$ and the inverse temperature $\beta \in \mathbb{R}^{+}$. Note that, in our study, we take the limit $L_{\mathrm{i}} \rightarrow \infty$ after $L_{\mathrm{f}} \rightarrow \infty$. See, for instance, Equation (106).

By [BR96b, Theorem 6.2.42 and discussions on page 294] (quantum spin systems) or [AM03, Theorems 6.4, 11.7, 12.11] (fermion systems), for any translation-invariant and finite-range interaction $\Phi_{\mathrm{f}}, \Phi_{\mathrm{i}}$, there is at least one translation-invariant KMS state $\rho \in E_{1}$. In this case, it is well-known that the set of all translation-invariant KMS states associated with $\left\{\tau_{t}\right\}_{t \in \mathbb{R}}$ and $\beta \in \mathbb{R}^{+}$is a weak*closed face of $E_{1}$ and thus, there is at least one such KMS state which is ergodic. In particular, the uniqueness of the KMS state automatically yields its ergodicity. This is the typical situation studied in previous results [LLS00, dRMNS15, LR05, O10].

\section{Remark 8.4 (LDP and uniqueness of KMS states)}

[NR04] is a study of LD in quantum spin systems at inverse temperatures $\beta<\beta_{0}$ done by "polymercluster expansions", without explicitly using the uniqueness of KMS states. For sufficiently small $\beta>0$, however, the KMS state is unique. See discussions of [NR04, Remark 2.14]. In [HMO07] LD associated with particular ergodic states (finitely correlated ergodic states) are studied in dimension 1. See also [O10]. In [OR11] the authors impose strong conditions on the state, which are known to be satisfied in concrete models only when the KMS state is unique. In [BdSPX], similar to [GLM02], we will consider weakly interacting fermions on the lattice at any inverse temperature $\beta \in \mathbb{R}^{+}$. In this situation, the uniqueness of KMS is generally unknown.

Acknowledgments: This work is supported by FAPESP (2017/22340-9) and CNPq (309723/20205), as well as by the Basque Government through the grant IT641-13 and the BERC 2018-2021 program, and by the Spanish Ministry of Science, Innovation and Universities: BCAM Severo Ochoa accreditation SEV-2017-0718, MTM2017-82160-C2-2-P. We are very grateful to Y. Ogata and L. Rey-Bellet for hints and discussions. Finally, we thank Zosza Lefevre for linguistic corrections.

\section{References}

[A68] H. Araki, On the Diagonalization of a Bilinear Hamiltonian by a Bogoliubov Transformation, Publ. RIMS Kyoto Univ. Ser. A 4 (1968) 387-412.

[A69] H. Araki, Gibbs States of a One Dimensional Quantum Lattice, Commun. Math. Phys. 14(2) (1969) 120-157.

[A70] H. ArAKI, On Quasifree States of CAR and Bogoliubov Automorphisms, Publ. RIMS Kyoto Univ. 6 (1970/1971) 385-442. 
[A74] H. ARAKI, On the equivalence of the KMS condition and the variational principle for quantum lattice systems, Commun. Math. Phys. 38 (1974) 1-10.

[A87] H. ARAKI. Bogoliubov Automorphisms and Fock Representations of Canonical Anticommutation Relations, Contemp. Math. 62 (1987) 23-141.

[A08] S. ADAMS, Large deviations for empirical measures in cycles of integer partitions and their relation to systems of Bosons, Analysis and Stochastics of Growth Processes, LMS, Oxford University Press, 148-172 (2008).

[ABK06a] S. ADAMS, J.-B. BRU AND W. KÖNIG, Large systems of path-repellent Brownian motions in a trap at positive temperature, Electronic Journal of Probability 11 (2006) 460-485.

[ABK06b] S. ADAMS, J.-B. BRU AND W. KÖNIG, Large deviations for trapped interacting Brownian particles and paths, Annals of Probab. 34 (2006) 1340-1422.

[ACK11] S. AdAms, A. Collevechio, AND W. KÖNIG, A variational formula for the free energy of an interacting many-particle system, Annals of Probab. 39(2) (2011) 683-728.

[AG98] M. Aizenman And G.M. Graf, Localization bounds for an electron gas, J. Phys. A: Math. Gen. 31 (1998) 6783-6806.

[AK08] S. ADAMS AND W. KÖNIG, Large deviations for many Brownian bridges with symmetrised initial-terminal condition, Probab. Theory Rel. Fields 142(1-2) (2008) 79-124.

[AW16] M. Aizenman And S. WARZEL, Random Operators: Disorder Effects on Quantum Spectra and Dynamics. Graduate Studies in Mathematics, Vol. 168. American Mathematical Society. 2016.

[AM03] H. ARAKI AND H. MORIYA, Equilibrium Statistical Mechanics of Fermion Lattice Systems. Rev. Math. Phys. 15 (2003) 93-198.

[B66] F. A. Berezin, The Method of Second Quantization (Academic Press, New York-London, 1966).

[BdSP13] J.-B. BRU AND W. DE SiqueIRA PEDRA, Non-cooperative Equilibria of Fermi Systems with Long Range Interactions Memoirs of the AMS 224(1052) (2013).

[BdSP15] J.-B. BRU AND W. DE SiqueIRA PEDRA, From the 2nd Law of Thermodynamics to the AC-Conductivity Measure of Interacting Fermions in Disordered Media, Math. Models Methods Appl. Sci. 25(14) (2015) 2587-2632.

[BdSP17a] J.-B. BRU AND W. DE SIQUEIRA PEDRA, Lieb-Robinson Bounds for MultiCommutators and Applications to Response Theory, SpringerBriefs in Mathematical Physics, Vol. 13 (2017).

[BdSP17b] J.-B. BRU AND W. DE SiqueIRA PEDRA, Universal Bounds for Large Determinants from Non-Commutative Hölder Inequalities in Fermionic Constructive Quantum Field Theory, Math. Models Methods Appl. Sci. 27(10) (2017) 1963-1992.

[BdSPX] J.-B. BRU AND W. DE SiQUeIRA PEDRA, General Theory of Weakly Interacting Fermions in Equilibrium, in preparation. 
[BGPS94] G. Benfatto, G. Gallavotti, A. Procacci, and B. Scoppola, Beta function and Schwinger functions for a many fermions system in one dimension. Anomaly of the Fermi surface, Comm. Math. Phys., 160(1) (1994) 93-171.

[Bo11] G. D. BouCH, Complex-time singularity and locality estimates for quantum lattice systems, Ph.D. thesis, Rutgers The State University of New Jersey. See also arXiv:1011.1875.

[Br04] J.-B. BRU, Large deviations for the local particle densities, Preprint DIAS-STP-04-13, (33 pages, 2004).

[BR96a] O. Bratteli And D.W. Robinson, Operator Algebras and Quantum Statistical Mechanics, Vol. I, 2nd ed. Springer-Verlag, New York, 1996.

[BR96b] O. Bratteli And D.W. Robinson, Operator Algebras and Quantum Statistical Mechanics, Vol. II, 2nd ed., Springer-Verlag, New York, 1996.

[Bry90] W. BRYC, Large deviations by the asymptotic value method. In M. Pinsky, editor, Diffussion Processes and Related Problems in Analysis, pages 447-472. Birkhäuser, Basel, Switzerland, 1990.

[Bry93] W. BRYC, A remark on the connection between the large deviation principle and the central limit theorem, Statistics \& Probability Letters 18(4) (1993) 253-256.

[BZ98] J.-B. BRU AND V.A. Zagrebnov, Exact Solution of the Bogoliubov Hamiltonian for Weakly Imperfect Bose-Gas, J. Phys. A : Math. Gen. 31 (1998) 9377-9404.

[BZ08] J.-B. BRU AND V.A. ZAGREBnOV, Large Deviations in the Superstable Weakly Imperfect Bose Gas, J. Stat. Phys. 133(2) (2008) 379-400.

[ClR88] W. Cegla, J. T. Lewis, G. Raggio, The Free Energy of Quantum Spin Systems and Large Deviations, Commun. Math. Phys. 118 (1988) 337-354.

[CT73] J. M. Combes And L. Thomas, Asymptotic behaviour of eigenfunctions for multiparticle Schrödinger operators, Comm. Math. Phys.34(4) (1973) 251-270.

[dRMnS15] W. De Roeck, C. Maes, K. NetočnỲ, And M. Schütz, Locality and nonlocality of classical restrictions of quantum spin systems with applications to quantum large deviations and entanglement, J. Math. Phys. 56 (2015) 023301.

[dRS17] W. DE Roeck AND M. S ALmhofer, Persistence of exponential decay and spectral gaps for interacting fermions. arXiv:1712.00977 (2017).

[D06] J. DEREZIŃSKI, Introduction to Representations of the Canonical Commutation and Anticommutation Relations, pp 63-143 in Large Coulomb Systems: Lecture Notes on Mathematical Aspects of QED, Volume 695 of the series Lecture Notes in Physics, SpringerVerlag Berlin-Heidelberg, 2006.

[dSP05] W. DE SiqueIRA Pedra, Zur mathematischen Theorie der Fermiflüssigkeiten bei positiven Temperaturen. PhD Thesis, University of Leipzig, 2005.

[dSPS08] W. De Siqueira Pedra And M. Salmhofer, Determinant Bounds and the Matsubara UV Problem of Many-Fermion Systems, Commun. Math. Phys. 282 (2008) 797-818.

[DSt89] J.-D. Deuschel And D. W. STroock, Large Deviations, American Mathematical Soc., Providence, Rohde Island, 1989 
[DZ98] A. Dembo And O. Zeitouni, Large Deviations Techniques and Applications, 2nd ed. Springer-Verlag, New-York (1998).

[Di73] J. Dieudonne, Treatise on Analysis, Volume III, Academic Press, New York, 1973.

[DLP89] T. C. Dorlas, J. T. Lewis, J. V. PulÉ, The Yang- Yang Thermodynamic Formalism and Large Deviations, Commun. Math. Phys. 124 (1989) 365-402.

[DLP93] T. C. Dorlas, J. T. Lewis, J. V. Pulé, The Full Diagonal Model of a Bose Gas, Commun. Math. Phys. 156 (1993) 37-65.

[DP87] N.G. DufFiEld And J.V. PulÉ, Thermodynamics of the B.C.S. model through large deviations, Lett. Math. Phys. 14 (1987) 329-331.

[E85] R. Ellis, Entropy, Large Deviations and Statistical Mechanics, New York, Springer 1985.

[EN00] K.-J. EngEL AND R. NAGEL, One-Parameter Semigroups for Linear Evolution Equations, Springer New York, 2000.

[EK98] D.E. Evans AND Y. KaWAHIGASHI, Quantum Symmetries on Operator Algebras (Oxford Mathematical Monographs, Oxford University Press, 1998)

[FKT02] J. Feldman, H. Knorrer and E. Trubowitz, Fermionic Functional Integrals and the Renormalization Group,, CRM Monograph Series, Volume 16 (American Mathematical Society, 2002).

[GLM02] G. Gallavotti, J. L. Lebowitz and V. Mastropietro, Large deviations in rarefied quantum gases, J. Stat. Phys. 108(5/6) (2002) 831-861.

[GM10] A. Giuliani And V. Mastropietro, The Two-Dimensional Hubbard Model on the Honeycomb Lattice, Commun. Math. Phys. 293 (2010) 301-346.

[GMP16] A. Giuliani, V. Mastropietro and M. Porta, Universality of the Hall conductivity in interacting electron systems, Commun. Math. Phys. (2016) DOI: 10.1007/s00220-0162714-8.

[HMO07] F. Hiai, M. Mosonyi, AND T. OGaWA, Large deviations and Chernoff bound for certain correlated states on a spin chain, J. Math. Phys. 48 (2007) 123301.

[JOPP11] V. Jaksic, Y. Ogata, Y. Pautrat, And C.-A. PILlet, Entropic Fluctuations in Quantum Statistical Mechanics. An Introduction, ArXiv e-prints (2011).

[JOPP12] V. JAKsic, Y. Ogata, Y. PaUtRat , C.-A. Pillet, Entropic fluctuations in quantum statistical mechanics. An Introduction, Quantum Theory from Small to Large Scales, Lecture Notes of the Les Houches Summer School: Volume 95, August 2010 , Oxford University Press, USA (2012), 213-410.

[L73] O. E. LANFORD, Entropy and equilibrium states in classical statistical mechanics, Statistical Mechanics and Mathematical Problems (A. Lenard, ed.), Lecture Notes in Physics, vol. 20, Springer Berlin Heidelberg, 1973, pp. 1-113 (English).

[LR05] M. LENCI AND L. ReY-Bellet, Large Deviations in Quantum Lattice Systems: OnePhase Region, J. Stat. Phys. 119 (2005) 715-746.

[LLS00] J. L. Lebowitz, M. Lenci AND H. Spohn, Large Deviations for Ideal Quantum Systems, J. Math. Phys. 41 (2000) 1224-1243. 
[Le86] J.T. LEWIS, The large deviation principle in statistical mechanics: an expository account. In: Stochastic mechanics and stochastic processes, Swansea Conference Proceedings 1986. Davies, I., Truman, A. (eds.). Lecture Notes in Mathematics. Berlin, Heidelberg, New York: Springer.

[LZ88] J. T. Lewis, J.V. Pulé, V. A. Zagrebnov, The Large Deviation Principle for the Kac Distribution, Helvetica Physica Acta 61 (1988) 1063-1078.

[NR04] K. NetoČNỲ And F. Redig, Large Deviations for Quantum Spin Systems, J. Stat. Phys. 117 (2004) 521-547.

[O10] Y. OgAtA, Large Deviations in Quantum Spin Chains, Commun. Math. Phys. 296 (2010) $35-68$.

[OR11] Y. OgATA AND L. ReY-Bellet, Ruelle-Lanford Functions and Large Deviations for Asymptotically Decoupled Quantum Systems, Rev. Math. Phys. 23 (2011) 211-232.

[P02] C.-E. PFister, Thermodynamical aspects of classical lattice systems. In In and out of equilibrium (Mambucaba, 2000), Progr. Probab., 51, Birkhäuser, Boston, 2002. pp. 393472.

[RS72] M. REed AND B. Simon, Methods of Modern Mathematical Physics, Vol. IV. Academic Press, New York-London, 1972.

[RB11] L. REY-BELLET, Large Deviations in Quantum Spin Systems, May 2011, http://www.math.harvard.edu/conferences/frg11/talks/rey-bellet.pdf.

[Ru65] D. Ruelle, Correlation Functionals, Journal of Mathematical Physics 6 (1965), 201-220.

[RW89] G.A. RAGGIO AND R.F. WERnER, Quantum statistical mechanics of general mean field systems. Helv. Phys. Acta 62 (1989) 980-1003.

[T09] H. Touchette, The Large Deviation Approach to Statistical Mechanics, Physics Reports 478 (2009), no. 1, 1-69.

[VlP88] M. Van den Berg, J. T. Lewis, J. V. Pulé, The Large Deviation Principle and Some Models of an Interacting Boson Gas, Commun. Math. Phys. 118 (1988) 61-85.

[VDlp90a] M. van den Berg, T. C. Dorlas, J. T. Lewis, J. V. Pulé, A Perturbed Mean Field Model of An Interacting Boson Gas and the Large Deviation Principle Commun. Math. Phys. 127 (1990) 41-69.

[VDlp90b] M. Van den Berg, T. C. Dorlas, J. T. Lewis, J. V. Pulé, The Pressure in the Huang-Yang-Luttinger Model of an Interacting Boson Gas, Commun. Math. Phys. 128 (1990) 231-245.

[V66] S.R.S. VARADhan, Asymptotic probabilities and differential equations, Comm. Pure Appl. Math. 19 (1966) 261-286.

[V11] A. Verbeure, Many-Body Boson Systems. Theoretical and Mathematical Physics Series, Springer-Verlag London, 2011.

[ZB01] V.A. Zagrebnov AND J.-B. BRU, The Bogoliubov Model of Weakly Imperfect Bose Gas. Phys. Rep. 350 (2001) 291-434. 